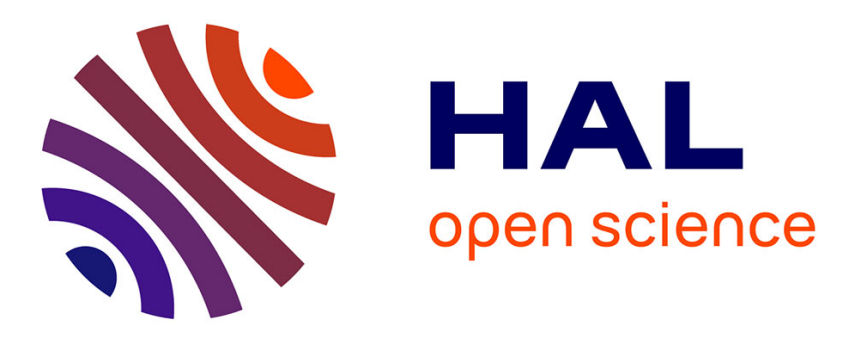

\title{
Review and Perspectives: Shape Memory Alloy Composite Systems
}

Brian Lester, Theocaris Baxevanis, Yves Chemisky, Dimitris Lagoudas

\section{To cite this version:}

Brian Lester, Theocaris Baxevanis, Yves Chemisky, Dimitris Lagoudas. Review and Perspectives: Shape Memory Alloy Composite Systems. ACTA MECHANICA, 2015, 10.1007/s00707-015-1433-0 . hal-01199415

\section{HAL Id: hal-01199415 https://hal.science/hal-01199415}

Submitted on 15 Sep 2015

HAL is a multi-disciplinary open access archive for the deposit and dissemination of scientific research documents, whether they are published or not. The documents may come from teaching and research institutions in France or abroad, or from public or private research centers.
L'archive ouverte pluridisciplinaire HAL, est destinée au dépôt et à la diffusion de documents scientifiques de niveau recherche, publiés ou non, émanant des établissements d'enseignement et de recherche français ou étrangers, des laboratoires publics ou privés. 


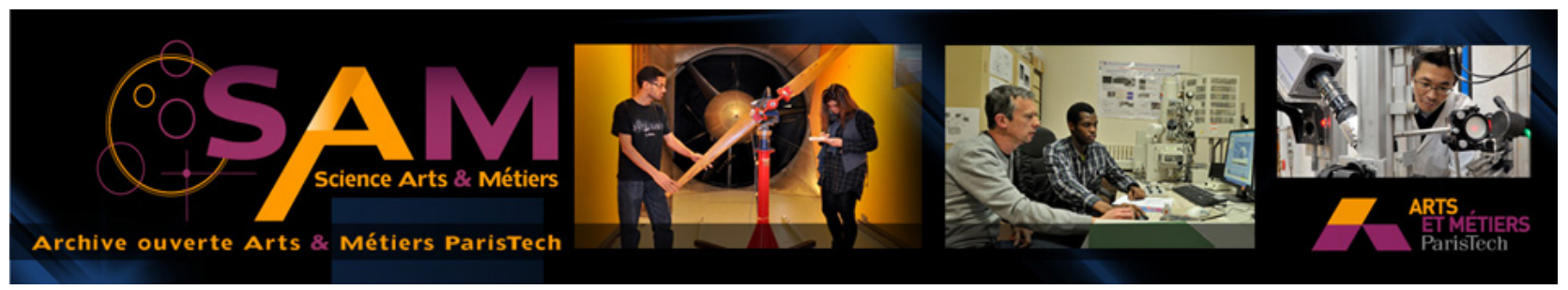

Science Arts \& Métiers (SAM)

is an open access repository that collects the work of Arts et Métiers ParisTech researchers and makes it freely available over the web where possible.

This is an author-deposited version published in: http://sam.ensam.eu

Handle ID: .http://hdl.handle.net/10985/10009

\section{To cite this version :}

Brian LESTER, Theocaris BAXEVANIS, Yves CHEMISKY, Dimitris LAGOUDAS - Review and Perspectives: Shape Memory Alloy Composite Systems - ACTA MECHANICA p.60p. - 2015 
Brian T. Lester . Theocharis Baxevanis • Yves

Chemisky · Dimitris C. Lagoudas

\title{
Review and Perspectives: Shape Memory Alloy Composite Systems
}

Received: date / Accepted: date

\begin{abstract}
Following their discovery in the early 60's, there has been a continuous quest for ways to take advantage of the extraordinary properties of shape memory alloys (SMAs). These intermetallic alloys can be extremely compliant while retaining the strength of metals and can convert thermal energy to mechanical work. The unique properties of SMAs result from a reversible difussionless solid-to-solid phase transformation from austenite to martensite. The integration of SMAs into composite structures has resulted in many benefits, which include actuation, vibration control, damping, sensing, and selfhealing. However, despite substantial research in this area, a comparable adoption of SMA composites by industry has not yet been realized. This discrepancy between academic research and commercial interest is largely associated with the material complexity that includes strong thermomechanical coupling, large inelastic deformations, and variable thermoelastic properties. Nonetheless, as SMAs are becoming increasingly accepted in engineering applications, a similar trend for SMA composites is expected in aerospace, automotive, and energy conversion and storage related applications. In an effort to aid in this endeavor, a comprehensive overview of advances with regard to SMA composites and devices utilizing them is pursued in this paper. Emphasis is placed on identifying the characteristic responses and properties of these material systems as well as on comparing the various modeling methodologies for describing their response. Furthermore, the paper concludes with a discussion of future research efforts that may have the greatest impact on promoting the development of SMA composites and their implementation in multifunctional structures.
\end{abstract}

Keywords Shape Memory Alloys · Composites · Micromechanics · Multiscale Modeling · Finite Element Analysis

\author{
B. T. Lester \\ Department of Aerospace Engineering, Texas A\&M University, College Station, TX 77843-3141, USA \\ E-mail: brian.t.lester@gmail.com \\ T. Baxevanis \\ Department of Aerospace Engineering, Texas A\&M University, College Station, TX 77843-3141, USA \\ E-mail: theocharis@tamu.edu \\ Y.Chemisky \\ Arts et Métiers ParisTech, LEM3-UMR 7239 CNRS, 4 Rue Augustin Fresnel, 57078 Metz, France \\ E-mail: yves.chemisky@ensam.eu \\ D. C. Lagoudas \\ Department of Aerospace Engineering and Department of Materials Science \& Engineering, Texas A\&M Uni- \\ versity, College Station, TX 77843-3141, USA \\ E-mail: lagoudas@tamu.edu
}




\section{Introduction}

Shape memory alloys (SMAs) are a unique class of intermetallic materials capable of undergoing a reversible solid-to-solid phase transformation via thermal and/or mechanical loadings which may result in large recoverable, inelastic strains of $\sim 1-8 \%[232,325]$. Specifically, at high temperature and low stress the material structure is that of a high-symmetry (cubic) austenitic phase while at low temperature and high stresses a lower symmetry (typically orthorhombic or monoclinic) martensitic structure is observed. Two key behaviors of SMAs result from this transformation - the shape memory effect (SME) and pseudoelasticity. The former refers to the ability of the material to recover large, seemingly permanent strains via heating from a deformed shape in martensite to a remembered, austenitic one while the latter is associated with SMAs being able to undergo large, hysteretic stress-strain excursions without any permanent deformations at a sufficiently high temperature [232]. Because of these exciting responses, SMAs have been increasingly investigated for application in roles such as actuators [46], couplers, and vibration dampeners [75], in the aerospace [172,74], civil [326,406,115], and petroleum [4] industries as well as in MEMS devices [45]. Furthermore, NiTi (the most common SMA) is biocompatible leading to the application of SMAs in biomedical devices such as stents $[286,118,302]$ and implants [31,123].

Since their discovery [72], SMAs have been increasingly accepted as an engineering solution to a wide variety of problems. As such, multiple research efforts have focused on expanding their potential applications [410] by exploring ways of taking advantage of or even improving their unique performance-related characteristics. To accomplish this goal, Ashby and Bréchet [16] identified two possible approaches: (i) the development of new alloys and material systems or (ii) the creation of hybrid materials that combine the characteristics of existing materials. Efforts towards the former have resulted in the creation of high-temperature SMAs (HTSMA) $[285,138]$, magnetic shape memory alloys (MSMAs) [211,359], and shape memory polymers (SMPs) [251,373]. The latter possibility, however, holds more excitement with respect to SMAs. Specifically, by combining one (or more) SMA phases with other constituents, novel material systems with both specific effective thermoelastic and transformation behaviors may be created. Such a concept incorporates materials with distinct phases including using SMA as either reinforcement or matrix and porous media. The former will be referred to (collectively) as SMA composites and efforts into this area started in the late 1980's, when Rogers and Robertshaw [383] first embedded NiTiNOL wires in a laminated polymer matrix composite (PMC). In this initial investigation, Rogers and Robershaw [383] first introduced the term SMA composite and classified this material as an adaptive material that they defined as, "... a composite material that contains shape memory alloy fibers (or films) in such a way that the material can be stiffened or controlled by the addition of heat." As will be seen in the remainder of this paper, the SMA composite class of materials has greatly expanded in terms of microstructure and application versus that original definition. Examples are shown in Fig. 1 in which micrographs of polymer, metal, and ceramic matrix composites and porous specimens are presented. The indicated composites exhibit a variety of reinforcement types (e.g., fiber, particulate, controlled channel networks) and have been considered for a wide range of purposes. A complete listing of composites that have been manufactured and used for either experimental or application purposes is presented in a later section for composites with the SMA playing the role of either the reinforcement or matrix.

Initial efforts into SMA composites focused on utilizing the SME of SMA wires in two ways - active property tuning and active strain energy tuning (commonly referred to in the literature as APT and ASET, respectively) $[383,382,267,379]$. Active property tuning refers to transformation of undeformed SMA wires from their martensitic state to the austenitic one to take advantage of the corresponding increase in elastic modulus which can be quite useful in vibration, damping, and structural control. For active strain energy tuning, initially elongated wires are heated back to their remembered, austenitic shape. The induced contraction of the wires leads to large internal stresses (and strain energy) that, in addition to the change in modulus, can provide even greater control over vibration, damping, or other structural characteristics [380,268,381,394,42,43,41,377,378,126]. Baz and Ro [44] extended this concept by accounting for the inherit energy dissipation of martensitic transformation in conjunction with changes in composite stiffness to achieve optimal vibration control over a broad frequency spectrum. The SME was further used to increase the effective yield strength in SMA/metal matrix composites due to the internal forces associated with thermal recovery as initially proposed by Yamada et al. [460] and demonstrated by Armstrong and Kino [8]. Paine et al. [332] also used the SME with a NiTi reinforced 


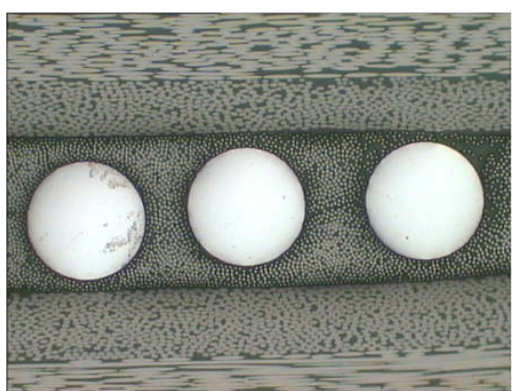

(a) Carbon/epoxy matrix reinforced with small diameter $(0.25 \mathrm{~mm}) \mathrm{NiTi}$ wires. Reprinted from [488] with permission from Elsevier.

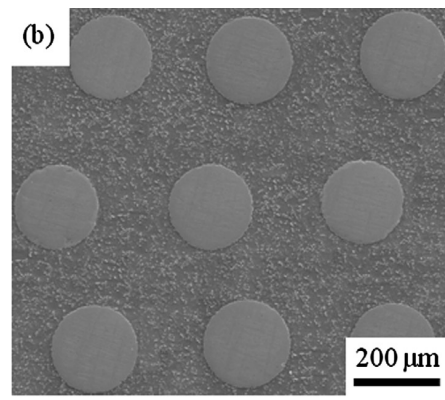

(d) Al matrix (reinforced with $5 \% \mathrm{SiC}$ particles) composite reinforced with long NiTi fibers. Reprinted from [183] with permission from Elsevier.

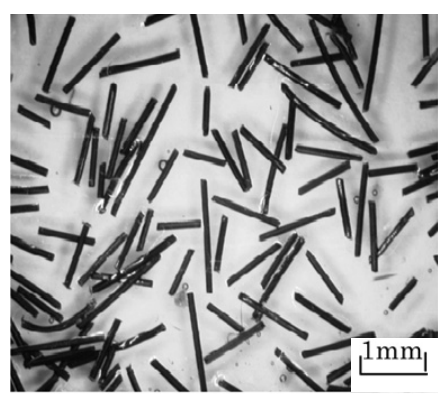

(b) ER3 epoxy resin matrix reinforced with $16.1 \mathrm{wt} \%$, dispersed short $(\approx 2 \mathrm{~mm}) \mathrm{NiTi}$ fibers. Reprinted from [320] with permission from Elsevier.

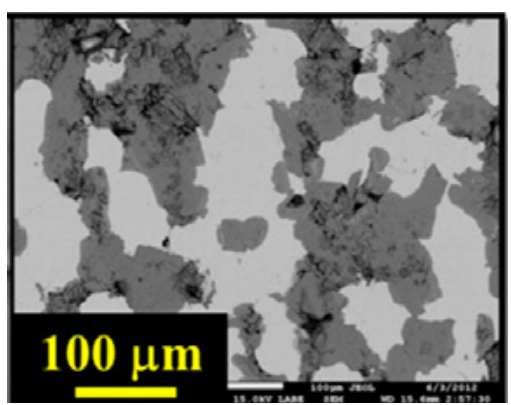

(c) $\mathrm{NiTi}-\mathrm{Ti}_{3} \mathrm{SiC}_{2}$ composite produced via spark plasma sintering. Reprinted from [187] with permission from Elsevier.

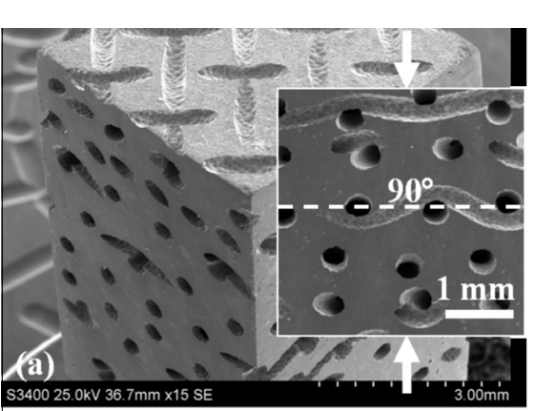

(f) Porous NiTi specimen with controlled 2D micro channels produced by via sacrificial steel meshes. Reprinted from [315] with permission from Elsevier.

Fig. 1: Examples of different SMA reinforced polymer (a,b), ceramic (c), and metal (d-e) matrix composite and porous (f) systems that have recently been developed.

polymer matrix system to relieve peak tensile stresses in pressure vessels. Other topics of the early investigations included improved impact damage resistance via the dissipative nature of the hysteric response of pseudoelastic SMAs [329,331], enhancement of critical buckling loadings and improvement in the post-buckling response [376]. Shape memory alloy reinforced composites were also considered as promising candidates for morphing structures. Composite actuators in the form of beams and rods were first analyzed by Chaudhry and Rogers [83] and Lagoudas and Tadjbakhsh [241,242], respectively, and subsequent efforts focused on characterizing the actuation response of SMA composites soon followed [449,33,60] (including SMA-piezoelectric systems [235, 236]). Over the years, actuators comprised of SMA composites have been extended from simple engineering configurations (e.g., rods and beams) to complex structures. Specifically, in the aerospace community, adaptive jet-engine chevrons [426, 170], variable twist UAV propellor rotors [339,340], and morphing wings [401] have all been developed. Similar principles have also been combined with origami concepts to create self-folding SMA composites capable of attaining a variety of configurations simply by changing the applied thermal loading $[349,350]$. Recent interest in bio-inspired applications has also led to a number of biomimetic robots. The motion of jellyfish [402,433,434] and turtles [221] (Fig. 2b) have been studied and used as a basis to develop SMA composite driven propulsive, aquatic machines. Locomotive devices based on inchworm movement has been used to create land-based robots [442]. Additional studies have also looked at applications beyond morphing structures including safety health monitoring devices (e.g., [355]). Thermally induced recovery loads have also been considered to improve self-healing [224] in polymers and damage suppression and toughening of concrete [264] (Fig. 2c). 


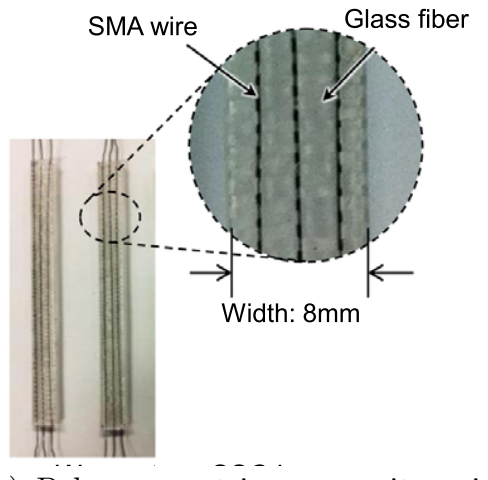

(a) Polymer matrix composite reinforced with smart SMA weave in insert. SMA wires are used as warp fibers while carbon fibers take the weft position thereby improving shape retention possibilities. (CIOP Publishing. Reproduced from [453] by permission of IOP Publishing. All rights reserved.

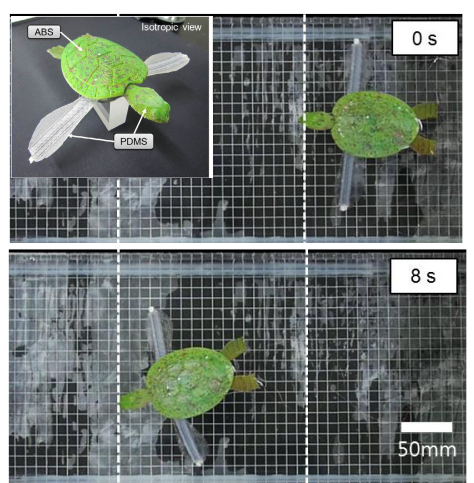

(b) Bioinspired turtle robot constructured using SMA composites as actuators for motion. Top and bottom show the start and finish of a swimming test with the robot displayed in the insert. (C)IOP Publishing. Reproduced from [221] by permission of IOP Publishing. All rights reserved.

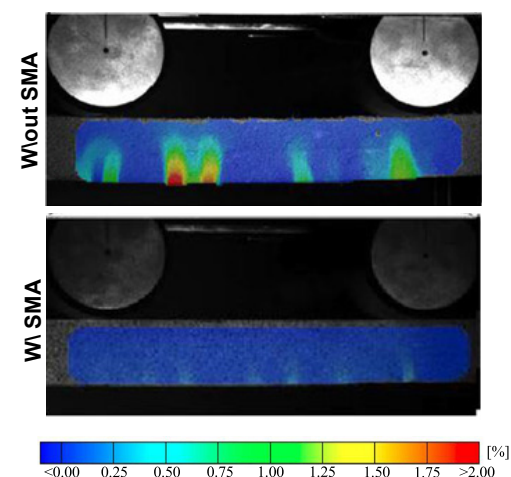

(c) Digital image correlation results of engineering cementitious composite (ECC) beams (508mm in length) with and without SMA reinforcement at the end of 7 loading cycles. Strain fields in the without specimen (top) indicate the presence of microcracks which are greatly diminished by SMA presence (bottom). (CIOP Publishing. Reproduced from [264] by permission of IOP Publishing. All rights reserved.

Fig. 2: Recent examples highlighting efforts investigating (a-b) SMA composite actuators and (b) damage recovery/healing via SMA reinforcement.

The development of these SMA composite applications has been enabled by extensive experimental efforts into the underlying mechanics. The influences and effects of large recovery strains associated with martensitic transformation were of particular interest. Such deformations were investigated with respect to processing of SMA/polymer composites, where normal cure cycles can induce recovery in the SMA, and interfaces to ensure sufficient strength to survive transformation. With respect to the former, Paine and Rogers [328] first studied the effect of processing temperatures on the nominal recovery stress in thermoplastic systems while Hebda et al. [175] investigated the impact of SMA properties and curing techniques and conditions on graphite/epoxy reinforced systems. With respect to the latter, pull-out experiments were performed to study a variety of surface treatments and demonstrated the early capabilities of sand blasting and the importance of mechanical interactions on interfacial strength $[327,205,203]$. Additionally, photoelastic experiments by Jonnalagadda and coworkers explored load transfer in systems with embedded SMA wires [203] and ribbons [204] and demonstrated that increased adhesion lowers wire displacement while increasing interfacial stress and transformation fronts move towards the center, respectively. Given the strong thermomechanical coupling exhibited by SMAs (notably, the latent heat of transformation), the thermal transport in these composites was also investigated [174,375]. Such studies into the underlying mechanics, along with application development, were substantially aided by various analytical [382,196,49], micromechanical [460, 237,68,69], and finite element $[174,42,237,240]$ modeling methodologies that were utilized to study these problems. Even with these initial and subsequent investigations, some of these first issues (e.g., interface damage and damage suppression) are still of interest for SMA composites - albeit for a larger set of material systems. For additional discussions of these early efforts, the interested reader is refered to the works of Paine and Rogers [330] and Wei et al. [448] as well as that of Birman [56] and Neuking et al. [314] who focused on some of the underlying mechanics issues and polymer matrix systems, respectively.

The present paper reviews and discusses the experimental and modeling advances on the manufacture, characterization, and simulation of SMA composites and devices utilizing them. Towards this objective, it is organized as follows. First, a brief review on the bulk SMA responses is given in Section 2 to aid in subsequent discussions. Recent efforts in manufacturing, experimentation, and application development of SMA composite systems are reviewed in Section 3 while Section 4 presents various 
efforts and approaches to model and analyze SMA composites. Finally, Section 5 discusses the current state of SMA composites with an eye towards topics that have not been addressed satisfactorily and concludes with a discussion of future research priorities that may strongly influence their development and applications.

\section{Characteristic Bulk SMA responses}

Since the discovery of NiTi as an SMA at the Naval Ordinance Laboratory (hence, NiTiNOL) in the early 60's [72], SMAs have received great interest for their unique thermomechanical behavior especially the aforementioned shape memory and pseudoelastic effects. A complete review of efforts investigating the metallurgical, crystallographic, and phenomenological issues associated with these responses is outside the scope of this work and the interested reader is referred to various texts [324, $232,325]$.

To aid in subsequent discussions, a brief review of the pertinent characteristics and behaviors of SMA transformation is presented. First, to describe when transformation occurs, transformation temperatures are typically utilized. Such temperatures refer to those observed under zero macroscopic loading. Specifically, the martensitic start, $M_{s}$ and finish, $M_{f}$, temperatures denote the initiation and completion of forward (austenite to martensite) transformation, respectively, while the austenitic start, $A_{s}$, and finish, $A_{f}$, are used for the initiation and end of reverse (martensite to austenite) transformation. These temperatures may be readily determined from calorimetry measurements as forward and reverse transformation are accompanied by either the release or absorption of heat, respectively, that is conventionally referred to as the latent heat of transformation [400,122,364,304]. Due to the thermomechanical nature of the martensitic transformation, as the applied stress increases so do the transformation temperatures. This coupling may be observed on a stress-temperature phase diagram like that in Fig. 3a via the linear dependence of the various transformation temperatures. The slopes of these lines are denoted as $C^{M}$ and $C^{A}$ for forward and reverse transformation, respectively, and are commonly referred to as stress-influence coefficients. Although linear lines in Fig. 3a, in multiaxial stress-temperature space these transformation limits form complex surfaces in three dimensions and are functions of loading path.

The shape memory and pseudoelastic responses may also be discussed via the phase diagram and are described in Fig. 3. First, upon cooling from the austenitic state the SMA will be in either a twinned or detwinned martensitic configuration depending on the applied macroscopic load. At lower loads, multiple martensitic variants may be present enabling the microstructure to self-accomodate via twinning. Macroscopically, the transformation from austenite to self-accomodated martensite produces no inelastic strain. If a sufficient load is applied during cooling or from the self-accomodated state, a detwinned structure is produced along with a corresponding macroscopic strain which may be recovered via heating and is referred to as the shape memory effect. The case of the heating-cooling cycle being performed under an applied load is commonly referred to as the actuation response and is indicated by path $(A)$ in Fig. 3a. The corresponding strain-temperature response under a constant applied loading is presented in Fig. 3b. The inelastic strain developed as a result of this loading path is commonly referred to as the actuation strain and is denoted $\varepsilon^{a c t}$ in Fig. 3b. Additionally, in the case of an isobaric loading, the mechanically applied stress is referred to as the actuation stress and the product of the actuation stress and strain is the actuation work density. Pseudoelasticity, on the other hand, refers to isothermal mechanical loading and unloading from above the zero-stress $A_{f}$ temperature thereby undergoing complete transformation (path $B$ in Fig. 3b). The distinct flag shape with two linear elastic regimes (with distinct elastic moduli $E^{A}$ and $E^{M}$ for the austenitic and martensitic phases, respectively) and recoverable transformation strain of magnitude, $H$, characteristic of the pseudoelastic response are readily apparent in Fig. $3 \mathrm{~b}$. It should be noted that in the actuation case, the actuation strain, $\varepsilon^{a c t}$, differs from the transformation strain of the pseudoelastic case in that is also has additional contributions associated with the change in thermoelastic properties of the phases.

The transformation of interest to SMAs also produces additional complexity to the response. For instance, during initial cycles, cyclic instabilities are typically observed resulting in the generation of irrecoverable plastic strains and permanent changes in the transformation hysteresis. These deformations arise from plastic accommodation being necessary in addition to transformation behaviors to satisfy compatibility constraints [226] although such strains saturate via repeated cycling. Often, this 


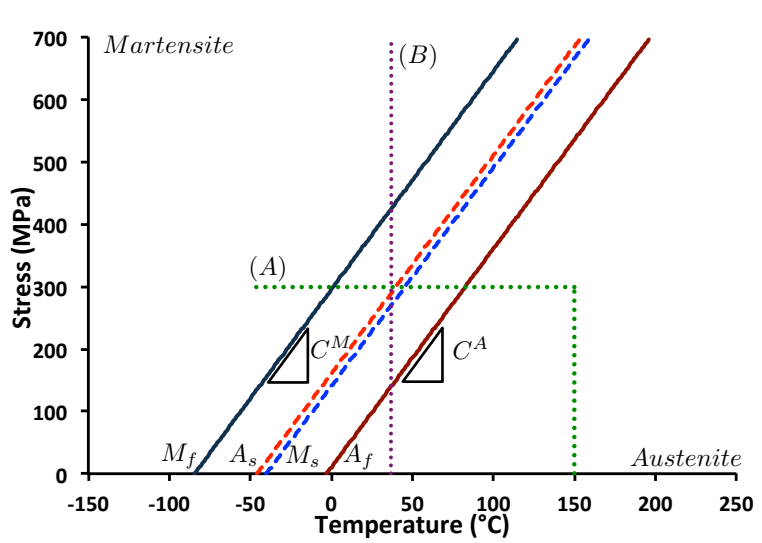

(a) Uniaxial stress-temperature phase diagram with transformation surfaces and loading paths for the actuation and pseudoelastic cases presented
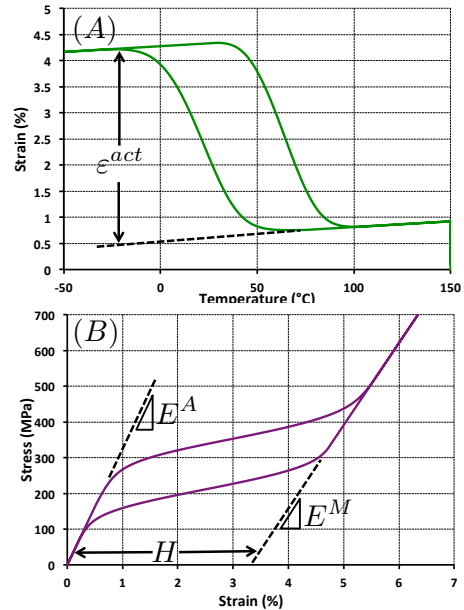

(b) (Top) Actuation (isobaric cooling heating cycles) response under a constant $100 \mathrm{MPa}$ load and (bottom) the pseudoelastic response

Fig. 3: Characteristics SMA behaviors: (a) phase diagram and (b) the corresponding responses. Presented results correspond to a $\mathrm{NiTi}(50.8$ at\% $\mathrm{Ni})$ material with properties given in Table 3 of Lagoudas et al. [233].

process is referred to as transformation induced plasticity (TRIP) and the corresponding strains are referred to as TRIP strains. Examples of this behavior with respect to both the pseudoelastic and actuation responses are presented in Fig. 4 in which clear variations in the response is noted over repeated cycling. First, a clear permanent deformation is developed in both cases which continues to grow with cycle. Furthermore, a clear change in transformation temperatures (or stresses in the pseudoelastic case) during cycling is evident. Additionally, and more clearly presented in the pseudoelastic case (Fig. 4a), the recoverable strain and hysteresis also reduce with further cycling but with a decreasing rate. TRIP strains induced by cycling also result in the generation of back (residual) stresses that give rise to a two-way SME (TWSME) in which a macroscopic actuation strain may be produced even under no external biasing loads. Such cases are primarily concerned with complete transformation cycles although a number of investigations have also studied the evolution of partial, internal transformation loops [412,152,61]. Finally, depending on the geometry, convective boundary conditions and associated heat transfer, the latent heat of transformation may have a strong impact on the deformation response and the stress-strain hysteresis loop area of SMAs as shown experimentally in [364] and is also responsible for apparent rate dependencies [400]. As such, the thermal response of SMAs in different configurations has been extensively investigated [288, 298,463].

In order to enable subsequent discussion and comparison with composite materials, some representative mechanical properties of different SMA alloys are given in Table 1.

Table 1: Properties of Selected Bulk SMAs (Data from Table 2.5 of [232]).

\begin{tabular}{l|cccr}
\hline Property & $\mathrm{NiTi}$ & $\mathrm{NiTiCu}$ & $\mathrm{NiTiPd}$ & $\mathrm{CuAlNi}$ \\
\hline$E^{A}(\mathrm{GPa})$ & 70 & 50 & 15 & 90 \\
$E^{M}(\mathrm{GPa})$ & 30 & 25 & 25 & 80 \\
$H(\%)$ & 6 & 5 & 3 & 4 \\
$\rho\left(\mathrm{kg} / \mathrm{m}^{3}\right)$ & 6500 & 6500 & 8200 & 7500 \\
\hline
\end{tabular}




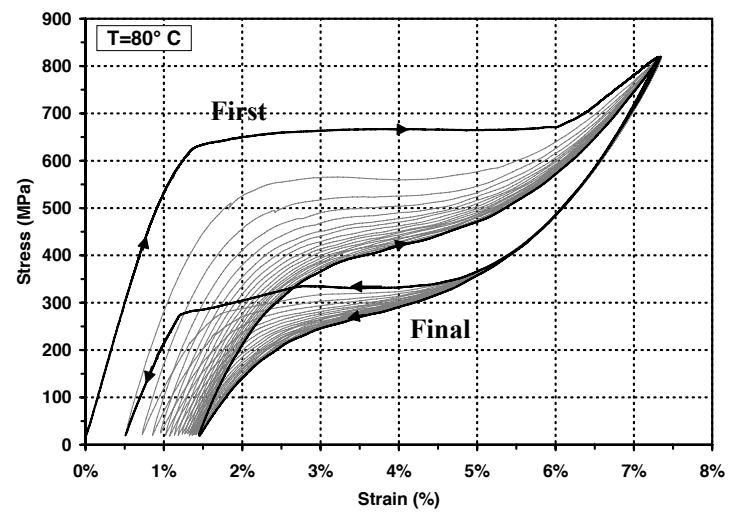

(a) Pseudoelastic response

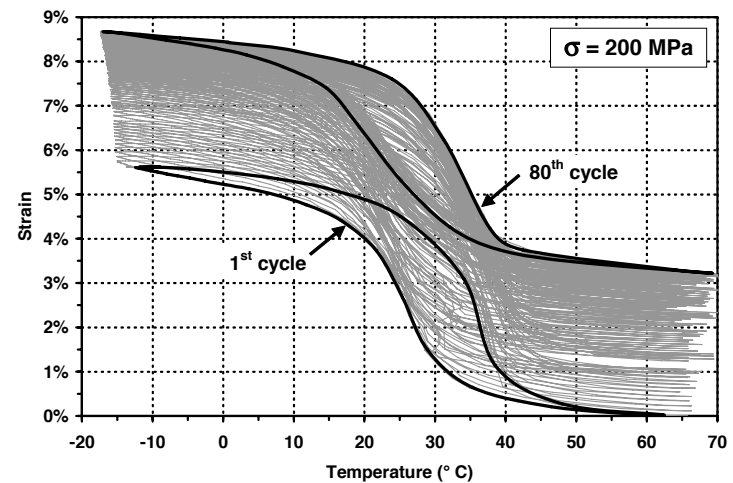

(b) Actuation response

Fig. 4: Evolution of characteristics SMA responses over repeated cycling (training).Taken from [169].

\section{Review of Processing Methods, Properties, and Applications of SMA Composites}

Heterogeneous SMA material systems considered in this work span SMA-reinforced polymers, metals, ceramics, and polymer composites as well as porous and reinforced SMA matrix materials. These material systems incorporate a broad set of properties, transformation characteristics, and responses that are discussed in the following section along with their fabrication and applications. Before proceeding to review each of the different composite types, however, it is important to consider the entire spectrum of materials in the context of material selection. Given their variety, the determination of the appropriate composite is often a complex task requiring multiple different criteria. A common way to tackle this problem is the utilization of material-property maps pioneered by Ashby [15]. In the case of SMA composites, both the actuation performance metrics and effective thermomechanical properties may be design drivers and examples of both are given in Fig. 5. To construct these maps, both experimental data and simple analysis bounds were utilized in constructing the respective composite domains indicated in these maps. In the analytical results, matrix property values were sampled from sets of representative material parameters from the Granta [157] material database and the fatigue limit values were used for estimating the actuation stress and strain values. For SMAs, the material properties of austenite were used in determining the composite modulus. First, as is commonly considered with active materials, the actuation energy density is given in Fig. 5a. The mass density versus actuation strain, actuation stress, and effective axial modulus are then presented in Fig. 5b-d, respectively. From Fig. 5a, it may be seen that many SMA composites retain energy densities comparable to that of the bulk SMA (the highest among conventional active materials). As shown in Fig. 5b-c, this work output is achievable in a variety of different combinations in multiple matrix systems opening up the possible design space. For instance, glass fiber-reinforced composite and elastomer matrix materials display similar actuation energies although the former occurs at higher stress and lower strains than the latter. Therefore, given the similar densities, selection of the different material class can enable the tailoring of actuation performance properties. With the composite nature of the materials, the effective elastic moduli may often be a design driver (e.g, matching bone stiffness as in the case of porous SMAs) and the corresponding map may be found in Fig. 5d. By taking these different maps in combination, a preferential material in terms of not only actuator performance but also with respect to effective composite performance. These maps will be revisiting in the following sections.

\subsection{Polymer Matrix \& Polymer Composite Matrix Composites (PM-SMAs and PC-SMAs)}

Two types of polymer-based composites are investigated in this section. The first is of polymer matrices reinforced by SMAs while the second is of polymer matrix composites (lamina or laminates of fiber reinforced polymers) reinforced by SMAs. In the literature, the latter case is sometimes referred to as SMA hybrid composites (SMAHCs). For clarity, "PM-SMAs" is used to refer to polymer matrices 


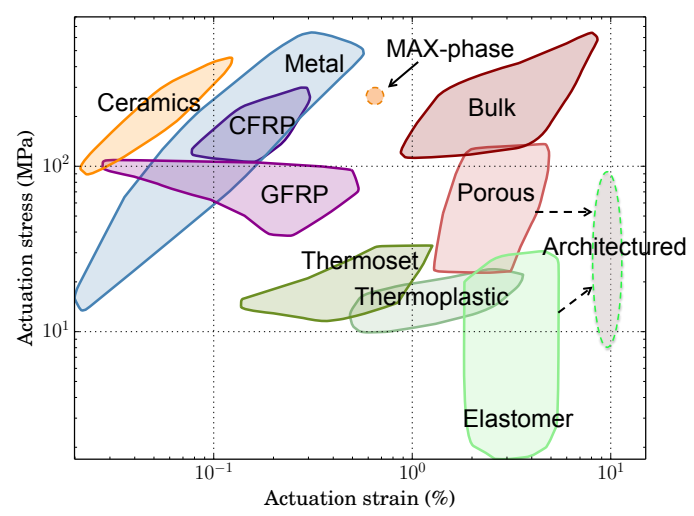

(a) Actuation energy density map.

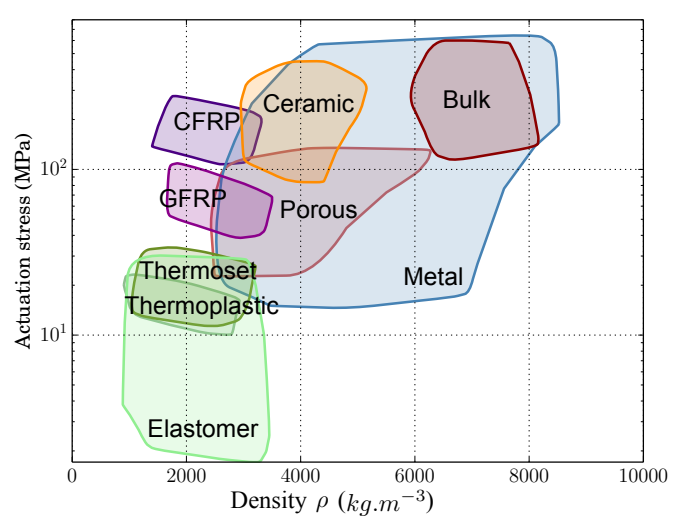

(c) Actuation stress versus mass density map.

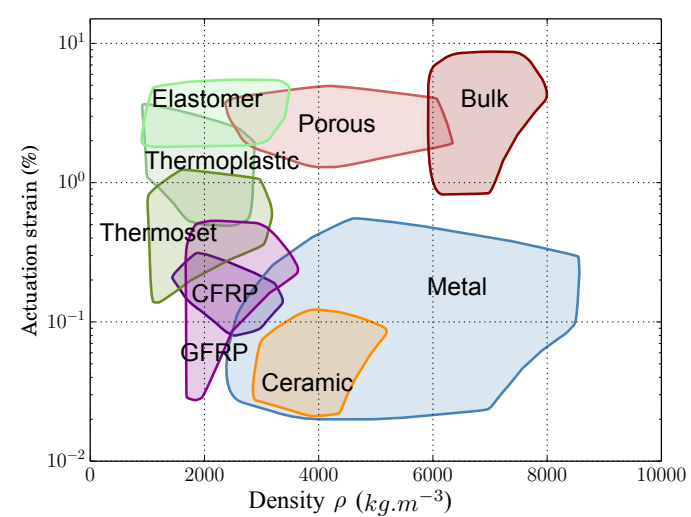

(b) Actuation strain versus mass density map.

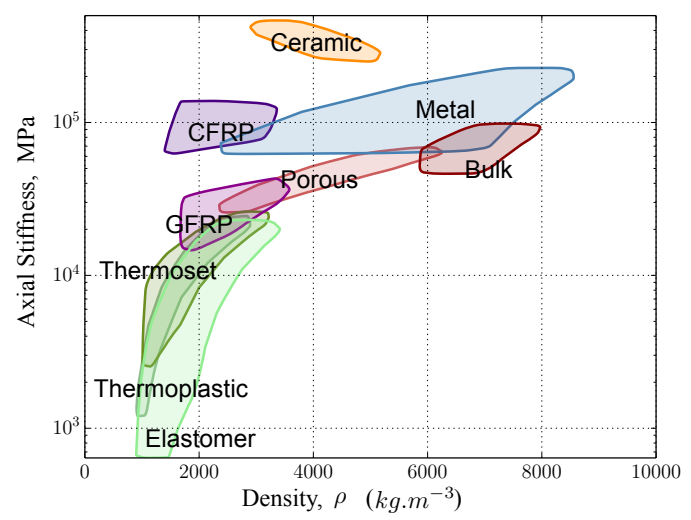

(d) Effective axial modulus versus mass density map.

Fig. 5: Material-Property maps highlighting actuation energy density, and mass density vs effective axial modulus, actuation strain, and actuation stress of heterogeneous and bulk, monolithic SMAs (denoted "Bulk") material systems. "Porous" refers to "Porous SMAs" while "GFRP" and "CFRP" representing glass and carbon reinforced polymers, respectively.

reinforced by SMAs while "PC-SMAs" denotes the case of polymer matrix composites reinforced by SMAs. The two classes will be collectively called "polymer-based". The use of the term "hybrid composites" shall be restricted to the more general case of a material system comprised of two or more distinct composites. Such composites have been investigated for a range of applications like actuators, increased damage resistance, and high-damping responses. This is enabled by the range of actuation performance metrics of these composite materials as shown in Fig. 5. Specifically, all of these materials have similar mass densities but have actuation stresses and strains spanning many decades. Similarly, they range from extremely compliant to having stiffnesses similar to the bulk or metallic cases given these material types extreme diversity. Recent efforts in these areas are presented in this section and a compilation of many of the polymer based composite systems that have been developed are given in Table 2 .

\subsubsection{Processing Methodologies Er Properies}

The effects of curing cycles and processing on the material state of SMA reinforced polymer-based composites has been an issue since the first reported systems $[328,175,49]$. A key issue of these cycles being the development of residual stresses induced through the curing process [49]. As SMA wires 
Table 2: Polymer Based Composites with SMA Reinforcement.

\begin{tabular}{|c|c|c|c|}
\hline \multicolumn{4}{|c|}{ PM-SMAs } \\
\hline Matrix Material & Reinforcement Type & Reinforcement Material & References \\
\hline ER3 Epoxy/Carbon PMC hybrid & Wire & $\mathrm{NiTi}$ & {$[474]$} \\
\hline ER3 Epoxy & Short Fiber & $"$ & {$[320,475]$} \\
\hline & Particulate & $"$ & {$[475]$} \\
\hline DGEBA Epoxy & Wire & $"$ & {$[80]$} \\
\hline Epon 828 Epoxy & $"$ & $"$ & {$[34]$} \\
\hline " & $"$ & $\mathrm{NiTiCu}$ & {$[224,222,318,317]$} \\
\hline Epon 828 Epoxy SMP & $"$ & $"$ & [414] \\
\hline Epon 826 Epoxy SMP & $"$ & $"$ & {$[151]$} \\
\hline E51 Epoxy SMP & $"$ & $"$ & {$[135,481]$} \\
\hline E-44 Epoxy & Particulate & $"$ & {$[417,418,416]$} \\
\hline D.E.R. 332 Epoxy & Wire & $"$ & [346-348] \\
\hline EC57/K21 Epoxy & $"$ & $"$ & [385] \\
\hline Epple 07170 Epoxy & Particulates & NiMnGa & {$[213]$} \\
\hline Hysol 9484 Epoxy & $"$ & $"$ & {$[165]$} \\
\hline Hysol 9455 Epoxy & $"$ & $"$ & [243] \\
\hline$"$ & Ribbon & $"$ & {$[243,165]$} \\
\hline$"$ & Bilayer & $"$ & {$[243]$} \\
\hline Hysol 9492 Epoxy & Particulates & $"$ & {$[243]$} \\
\hline & Ribbon & $"$ & {$[165]$} \\
\hline Araldite LY 3297 Epoxy & Fiber & $"$ & {$[154,153]$} \\
\hline Araldite-CY230 Epoxy & Wire & $\mathrm{NiTi}$ & {$[428]$} \\
\hline Epoxy & $"$ & $"$ & {$[360]$} \\
\hline Toughened Epoxy & Particulates & NiMnInCo & {$[271]$} \\
\hline Vinyl Ester & Wire & $\mathrm{NiTi}$ & {$[372]$} \\
\hline Polyester & Particulates & NiMnGa & {$[397,180]$} \\
\hline$"$ & $"$ & NiMnInCo & {$[273]$} \\
\hline$"$ & Ribbon & $"$ & {$[273]$} \\
\hline PDMS & Wire & $\mathrm{NiTi}$ & {$[225]$} \\
\hline$"$ & Wire (Warp Thread) & $"$ & {$[453]$} \\
\hline Silicone Rubber & Wire & $"$ & {$[388,374]$} \\
\hline Loctite 5140 Silicone & Particulates & NiMnGa & [243] \\
\hline Polyurethane (UR5048) & " & $"$ & {$[213]$} \\
\hline Polyurethane (Lord) & $"$ & $"$ & {$[136,137]$} \\
\hline Polyurethate & Particulates & $"$ & {$[287]$} \\
\hline Polyurethane SMP & Ribbons & $\mathrm{NiTi}$ & {$[419,421,422,420]$} \\
\hline Veriflex SMP & Wire & $"$ & [101] \\
\hline$"$ & Ribbon & $"$ & {$[250]$} \\
\hline PVDF & Bilayer & NiMnGa & {$[473]$} \\
\hline PMMA & " & $"$ & {$[403,164]$} \\
\hline PA6 Polyamide & Ribbon & $"$ & [313] \\
\hline TPO & Wire & $"$ & {$[6]$} \\
\hline \multicolumn{4}{|c|}{ PC-SMAs } \\
\hline PMC Reinforcement & SMA Type & SMA Material & References \\
\hline Carbon & Wire & $\mathrm{NiTi}$ & {$[20]$} \\
\hline$"$ & $"$ & $"$ & {$[195,7,488,338]$} \\
\hline Carbon/Aramid Hybrid & $"$ & $"$ & {$[356]$} \\
\hline Kevlar & Wire & $\mathrm{NiTi}$ & {$[214]$} \\
\hline & $"$ & $\mathrm{NiTiCu}$ & {$[485,486,63,425]$} \\
\hline Glass & $"$ & $"$ & {$[488,208,336,338,214]$} \\
\hline & & & $\&[132,411,308,404]$ \\
\hline$"$ & $"$ & $\mathrm{NiTiCu}$ & {$[486]$} \\
\hline$"$ & Ribbon & $\mathrm{NiTi}$ & {$[111]$} \\
\hline$"$ & " & $\mathrm{NiTiCu}$ & {$[9]$} \\
\hline
\end{tabular}

may undergo reverse transformation and associated inelastic deformations during the cure cycle, a mechanical frame is often used to pre-strain the fibers. Such a constraint can lead to cumbersome 
manufacturing processes and the development of substantial residual stresses. Recent efforts have included the development of a post-cure cycle eliminating the need for such a frame by ensuring SMA wires do not undergo reverse transformation [223]. Kirby et al. [223] studied the effect of these cycles on the residual strain and pull-out strength of the composite. Alternatively, Faiella et al. [132] utilized a vacuum infusion process to avoid reverse transformation during curing. To consider the load transfer in such cases, Digital Image Correlation (DIC) techniques have been used to determine the strain field in an SMA fiber embedded in an epoxy matrix [307] while Raman spectroscopy and thermography have been used to quantify residual stress fields in a fiber embedded in a PC-SMA composite [63]. With respect to NiTi reinforced glass fiber composites, Jang and Kishi [195] characterized the strength of the resultant composites as a function of orientation. To expand on potential capabilities of SMA polymer-based systems, novel wire architectures have also been investigated. Specifically, Heller and colleagues [176] investigated the response of an SMA composite with elastomer matrix reinforced with tubular braided NiTi wires under various loadings including tension and vibration tests as well as thermal cycles. It was shown that such composites maintained the SMA characteristics of NiTi while showing improved recoverable strain, load bearing capacity, and pseudoelastic damping versus single fiber composites. The potential of creating a smart weave with SMA wires as the warp threads and glass as weft has been investigated by Wu and coworkers [453] (as shown in Fig. 2a). By embedding such a configuration in a PDMS matrix, $76 \%$ of the actuated deflection may be retained even after the removal of the heating load. Arnaboldi et al. [9] considered removing elliptic patterns from a NiTiCu ribbon embedded in a glass fiber composite matrix to improve adhesion and demonstrated the improved dissipation characteristics of such a configuration.

In addition to effects of the cure cycle, bonding and adhesion of SMA constituents to matrix material through large deformation and temperature changes continues to present a critical issue. Building off of previous work focusing primarily on mechanical surface treatments [327,205,203], Neuking and coworkers [313] investigated the effect of different combinations of mechanical, chemical, and physical processing steps on the adhesion of a NiTi ribbon and polyamide matrix. In their work, a four step treatment was identified which produced an improved adhesion. Increases in adhesion strength (of over $100 \%$ in some case) via the utilization of different functionalization treatments have been reported [403, 164,6]. Surface engineering approaches, through engineered microgeometries [6], primer/plasma treatments [374], and depositing ZnO "nails" on the surface [481] have all been explored and the latter "nail" approach showed a 36\% improvement on the interfacial strength [481]. Sadrnezhaad et al. [388] and Rossi et al. [385] studied the effect of different mechanical and chemical surface treatments of NiTi wires on the interfacial shear strength in silicone and epoxy matrices, respectively. One of the novelties of SMAs with respect to interface adhesion is the variation in phase and thermoelastic properties with temperatures. To this end, Poon and coworkers conducted pull-out tests at different temperatures and constructed phase-stress-displacement diagrams (PSDD) to aid in accounting for this influence [360]. Payandeh et al. $[346,347]$ determined the effect of martensitic transformation on the interfacial shear strength of NiTi wire/epoxy matrix composites. It was shown that the interfacial shear strength increases when martensitic transformation/reorientation takes place in the SMA wires. A recent effort by Barrie and collaborators [34] sought to quantify this effect by a series of pull-out tests using heattreatments to vary the phase and transformation characteristics of the SMA wire embedded in an epoxy matrix. From these investigations, they concluded that austenite has a higher pull-out load than martensite regardless of whether the material transforms and that the elongation associated with transformation is the driver behind debonding.

The SMA reinforced polymer-based composites developed through these methods incorporate matrices that range from more compliant (e.g., elastomer) than the SMA reinforcement to comparable stiffnesses as in the case of various polymer composite matrix systems. As such, a range of responses may be observed from the developed composites. Some representative responses are presented below in Fig. 6. Specifically, in Figs. 6a and b the stress-strain and strain-temperature, respectively, responses of the braided NiTi composites developed by Heller et al. [176]. The temperature dependent response of an epoxy reinforced composite is presented in Fig. 6c demonstrating the temperature dependent nature of these materials while the recovery stress of a Kevlar fiber PC-SMA composite is given in Fig. 6d. Reference effective composite properties of various polymer-based composites are given in Table 3. 


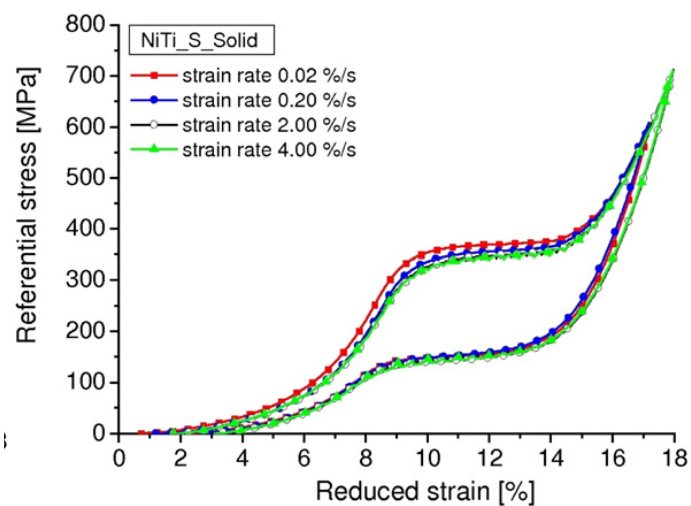

(a) Stress-strain response of a braided, solid NiTi-elastomer composite at different strain rates. (C)IOP Publishing. Reproduced from [176] by permission of IOP Publishing. All rights reserved.

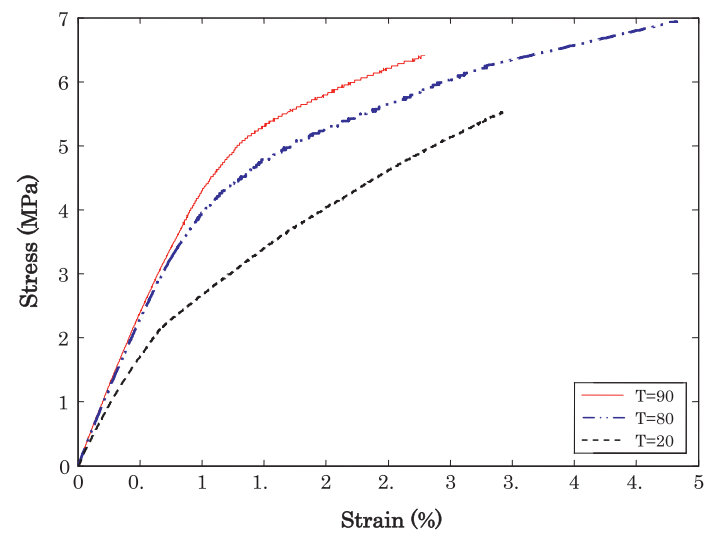

(c) Stress-strain response of a NiTi wire-epoxy composite at different temperatures (wire $A_{f}$ is $65^{\circ}$ C). Reprinted from [348] with permission from Elsevier.

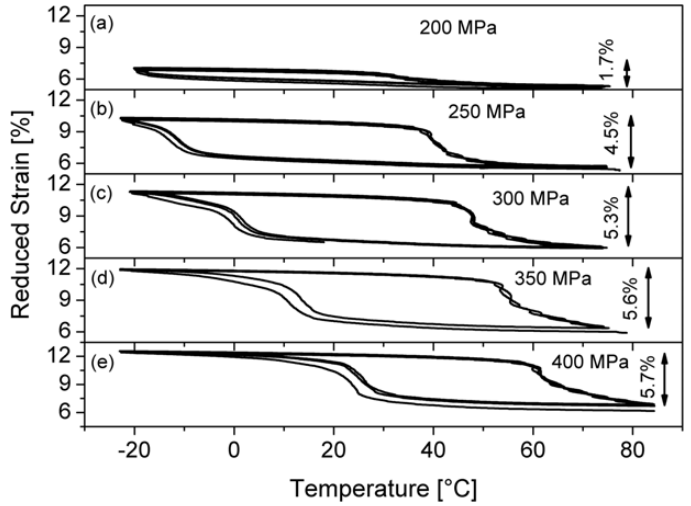

(b) Actuation response (strain-temperature under a constant stress), under different applied loads of a braided, hollow NiTi-elastomer composite. CIOP Publishing. Reproduced from [176] by permission of IOP Publishing. All rights reserved.

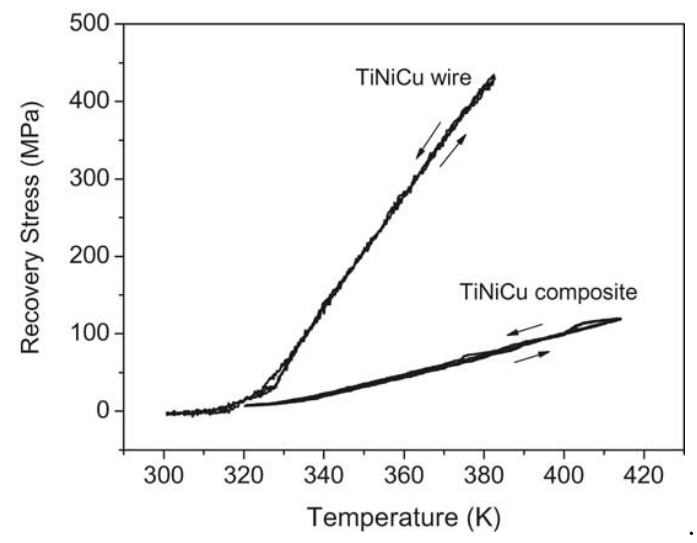

(d) Recovery stress generated by a $\mathrm{TiNiCu}$ wire and a Kevlar fiber composite reinforced with $11.8 \% \mathrm{TiNiCu}$ wires. In both cases, the wires are restrained to $3 \%$. Reprinted from [486] with permission from Elsevier.

Fig. 6: Characteristic responses of SMA reinforced polymer-based composites.

Table 3: Room Temperature Properties of Selected SMA Reinforced Polymer and Polymer Composite Matrix Systems.

\begin{tabular}{l|ccc}
\hline Property & Ester [372] & Hybrid SMA-Glass Fiber [336] & SMA-Glass Fiber [336] \\
\hline SMA vol\% & 20 & 1 & 1 \\
Modulus (GPa) & 12 & 16 & 6 \\
Strength (MPa) & 128 & 513 & 171 \\
Failure Strain (\%) & 12 & 4 & 7 \\
\hline
\end{tabular}

\subsubsection{Applications (PM-SMAs)}

Actuation performance and response - Although polymer composite matrix systems have been more extensively explored for use in actuators, SMA reinforcement in polymer matrices often provide useful comparison systems for studying the transformation responses of polymer composite matrix systems. For instance, Raghavan et al. [372] investigated the tensile and damping responses while three 
point bending tests have been performed to characterize short-fiber and particle reinforced epoxy systems $[475,320]$. Thermal profiles resulting from resistive heating of SMA wires and the impact of their spacing have also been experimentally determined. [428]. New applications have also been developed utilizing the actuation characteristics of SMA-polymer systems. In a new wrinkle, the use of SMA wires embedded in polymeric matrices has been studied for use in active texture control [225,81]. Specifically, Ko and coworkers [225] have explored embedding shape-set NiTi wires in a PDMS matrix and using the martensitic transformation as a way for active control of surface corrugation to improve surface characteristics like adhesion and friction.

To expand on the actuation possibilities, composites with shape memory polymers (SMPs) matrices have been developed. Such applications leverage SMPs that are an exciting class of polymers that can store large deformations through thermomechanical loading associated with changing from a low modulus rubbery phase at high temperature to a much stiffer (two orders of magnitude) "frozen" phase below its glass transition temperature [251,373]. Thus, by combining SMPs and SMAs exciting composites with enhanced multifunctionality may be created - notably with multiple, stable configurations. To explore this effect, Tobushi and coworkers have undertaken a series of experimental investigations [419, $421,422,420,423]$. Initial investigations focused on characterizing the bending response of a single SMA fiber composites and indicated that such a configuration may result in nearly $100 \%$ shape recovery while retaining $93-94 \%$ of the associated force $[419,421]$. Tensile testing at multiple temperatures have also indicated near perfect $(>98 \%)$ shape fixity and recovery at both high and low temperatures [135]. Enhancements in the behavior of these shape memory composites (SMC) belts were then investigated by using combinations of NiTi tapes and SMP matrices [422,420]. Specifically, SMA tapes heat treated to exhibit separately the pseudoelastic and shape memory effects at room temperature were shape set with round configurations. The two materials were then placed between layers of an SMP material - although facing in opposite directions. Through this combination, the SMCs was able to exhibit a "three-way actuation" by heating between the respective transformation temperatures of the SMAs and the glass transition of the SMP phase [422,420]. Taya and colleagues [414] have also manufactured an SMA-SMP composite and have reported a significant reversible deflection $(1.3 \mathrm{~mm}$ for a $10 \mathrm{~mm}$ span specimen) measured via three point bending testing. Thermal management becomes an important issue in these multistable configurations. Therefore, a recent effort by Lelieveld and Jansen [250] focused on identifying preferential heating paths in their architecturally focused composite. In a novel twist, Cortes and colleagues [101] used two-way trained SMA tubes as reinforcement in an SMP matrix and passed hot or cold fluid through the tubes to aid in composite temperature control. Manzo and Garcia [289] have also extended this concept by proposing an active joint comprised of SMA face sheets encompassing a SMP core and developed an analytical model to explore the response. A recent investigation has also explored the design considerations of SMA-SMP composites and demonstrated various applications for different configurations [151].

Self-healing - Recently, self-healing materials have been developed that utilize embedded healing agents and catalysts to repair cracks formed in an epoxy matrix [59]. Incorporation of SMA wires transverse to the crack direction has been proposed as way to improve the healing performance by using the SME to apply compressive forces on the crack face aiding curing and decreasing the associated repair volume $[224,222,318]$. Initial investigations demonstrated an increase in the healed peak load by cracking a specimen, inserting a healing agent and then activating the SMA wires [224]. Subsequent investigations focused on incorporating microencapsulated healing agents in the matrix and increases of up to $33 \%$ in the healed peak load were noted [222]. Furthermore, the extension of this concept to fatigue loadings has also been considered [317].

Improved damping - Incorporating SMA reinforcement into polymer matrices has long been explored to increase the damping behaviors due to the dissipative characteristics of martensitic transformation. With respect to polymer matrix systems, the damping properties of continual fiber [372, 80], short fiber [475,320], and particulate reinforced composites [475] have been characterized through dynamic mechanical analysis (DMA) experiments. More than 6 fold gains to the storage modulus over that of the matrix were reported with small (3.5 wt\%) additions of SMA content [320]. Particle reinforcement was also noted to have a higher loss factor than short fiber cases [475]. In the case of long fiber reinforcement, a higher storage modulus was reported with increasing NiTi wire content [80].

An extension of that concept is the consideration of magnetic shape memory alloys (MSMAs) embedded in polymer matrices. Specifically, MSMAs have been investigated due to their substantially higher actuation rate compared to conventional SMAs that are currently limited by thermal transport. 
Two mechanisms have been experimentally observed and have been investigated in these materials - magnetic domain reorientation [216,212] and field induced phase transformation (FIPT) [210,162]. With respect to the former (and more common mechanism), magnetic field induced strains (MFIS) may be generated under a mechanical bias load (although typically quite low, $\sim 6-10 \mathrm{MPa}[217]$ ). In single crystals, the MFIS may be up to $10 \%$ but very limited MFIS is typically observed in polycrystalline materials as grain boundaries suppress the twin motion responsible for the MFIS [120]. Both the introduction of porosity into polycrystalline specimens (Section 3.4) and MSMA as reinforcement in composites have been suggested to address this issue. Earlier reviews of Dunand and Müllner [120] and Liu et al. [272] delve into discussions of the various MSMA forms (including composites) and highlight these issues.

Recent efforts have focused on the improved damping in MSMA reinforced polymer composites. First, with respect to the magnetic domain reorientation, the response of NiMnGa reinforced polymer systems has been extensively studied. In such composites, utilization of a matrix with stiffness matched to that of the particulates is essential as: (i) twin boundary motion (which has been experimentally observed via neutron-diffraction [137]) is suppressed if the matrix response is too stiff $[243,165]$ and (ii) with soft materials, particulate motion and/or localized yielding arise and should be avoided [243, 213]. Similarly, good bonding between matrix and inhomogeneity is also needed and both pull-out [213] and single fiber fragmentation tests [153] have been performed indicating improved performance by using silane treatments $[213,153]$. Beyond the bonding, multiple investigations have also demonstrated the need for magnetically aligning the particles via an external field during curing produces an easy magnetic axis [397,180], thermally-induced transformation strain [418], and macroscopic MFIS [416] at the cost of some bending performance [417]. The damping characteristics have been measured through DMA [243,165], tension/compression tests for loss ratios [136], acoustic attenuation [287], and internal friction [416] and shown improved dissipation characteristics versus a variety of comparison materials. The possibility of FIPT has not been explored as extensively, but Liu and coworkers have studied embedding NiMnInCo particles in polyester [273] and epoxy [271] and demonstrated the occurrence of FIPT through multiple characterization techniques including the possibility of recovering pre-strains through magnetic stimuli [271]. Although most efforts have focused on particle reinforced systems, other architectures have been explored. For instance, NiMnGa wires were used as reinforcement in an epoxy system and demonstrated improved damping over a pure polymer system [154] while a NiMnGa/piezoelective PVDF bilayer was shown to have a large magnetoelectric effect [473].

\subsubsection{Applications (PC-SMAs)}

Actuation performance and response - SMA composites, especially those with polymer composite matrices, have long been considered as actuators and their shape memory and transformation characteristics under a variety of loading conditions has thus been characterized. For instance, the response of PC-SMAs in various configurations under both tensile $[411,348]$ and bending $[7,111,488,387,206]$ loadings have been extensively explored. Three point bending tests have been performed on composites with SMA wire reinforcement [7]. Thermal profiles resulting from resistive heating of composites beams through thermal cycles $[7,111,488]$ have also been characterized through various methods. Davis et al. [111] performed a coupled experimental (incorporating full field measurements) and modeling investigation of the response of polymer matrix composites with SMA ribbons under clamped conditions. Importantly, a strong dependence on initial geometric imperfections on the thermal buckling response was observed and reported. Wire reinforced composites under cantilever conditions were also considered [7,488]. Araujo and colleagues [7] noted the effect of SMA wire treatment on the composite response while Zhou and Lloyd [488] considered the impact of repeated cycles. From these efforts, delaminations were noted after several cycles limiting the potential multi-cycle capabilities of the composites considered. Essential for bending applications, the radius of curvature of SMA composites induced through reverse transformation has also been determined $[387,206]$. Specifically, in one case, silicon rubber inserts were added to the composite and the trade-off between bending deflection and stiffness was noted [387]. In relation to composite design, the role of fiber configuration (e.g., distance from mid-plane, wire spacing, and composite thickness) on the resulting bending performance of a PC-SMA composite was also characterized [206]. To aid in application implementation, the effective curvature limits of SMAHCs were determined via a series of experiments and modeling endeavors to identify the maximum possible transformation strain in different configurations [308]. Other efforts to 
this end looked at characterizing the effective actuation response of SMA reinforced composites [485, 486,425]. Specifically, Zheng et al. [485-487] measured the recovery stresses in a NiTiCu composite through thermal cycles (see Fig. 6d) and noted the impact of pre-strain and cycles on the response during recovery. Triantafyllou and Psarras [425] also looked at the reverse (recovery) transformation of a constrained $\mathrm{NiTiCu}$ composite via through electrical resistivity measurements. In this work, different pre-strain levels were considered and an increase in critical transformation temperatures with pre-strain magnitude was noted.

Damage suppression - SMA fiber reinforcement as a means to improve the damage suppression against impact loadings has long been considered in laminated composites [329,331]. An extensive review of this concept and some recent efforts may be found in the work of Angiono et al. [5]. Recent experimental efforts have built upon these previous investigations. Specifically, Kang and Kim [208] performed impact tests on a PC-SMA composite at different temperatures and determined the influence of induced damage through subsequent three point bending tests. In considering NiTi wires in both carbon and glass fiber matrix composites, Pappadà and coworkers [338] noted the possibility of NiTi wire reinforcement serving as damage initiation sites. The dissipated energy and peak load of a polymer matrix composite with pseudoelastic $\mathrm{NiTi}$ wire reinforcement were measured at different impact loads $[356,20]$ and a more than $100 \%$ improvement in dissipated energy was observed at the highest impact energy [20]. The impact response of a carbon fiber reinforced polymer matrix SMA composite at various impactor loads was also measured and compared to numerical results [296]. Additionally, a decrease in the area damaged was noted for composites with SMA wire reinforcement via non-destructive inspection techniques [337] and incremental loading experiments [404]. The ability of SMA reinforced fiber reinforced composites to serve as patches and repair damage was investigated by Khalili et al. [214] who demonstrated improved performance (in terms of impact and tensile performance) of a notched specimen repaired with SMA reinforced patches.

Improved vibration control - The vibration characteristics of PC-SMAs have been experimentally explored $[474,336]$. Zhang et al. [474] noted that vibration properties were more sensitive at higher temperatures where transformation could be induced. The same authors also noted that increasing the SMA content and accurate control over the developed internal stresses could lead to better tuning properties. Different SMA wire configurations (straight versus knitted) were studied by Pappada and coworkers [336] through laser vibrometry and impact tests and observed an increase in the damping properties.

Structural health monitoring - Qiu and colleagues [370] embedded an array of pseudoelastic wires in an glass fiber/epoxy composite and monitored the resistance of different SMA fibers to assess the location of loading and/or damage. Similarly, in a recent effort by Pinto et al. [355] the change in resistance of NiTi wire reinforcement and full field thermography were used to detect damage from impact or other loadings. Fiber Bragg Grating (FBG) sensors simultaneously measuring strain and temperature have been developed and utilized to measure the response of PC-SMAs [466] and study the impact of asymmetric lay-ups through thermal loadings [181].

\subsection{Metal Matrix Composites (MMCs)}

Initially, SMA reinforcement in metallic matrices (notably, Al) was considered for improving the effective yield strength through internal stresses induced through the SME. Given the variety of potential metal matrices, this composite class encompasses a wide range of actuation stresses and strains (Fig. 5cd) with similar diversity in terms of densities. As such, novel matrix materials and SMA reinforcement have been investigated and are detailed in the forthcoming sections. The various MMCs developed to this end are listed in Table 4.

\subsubsection{Processing Methodologies 83 Properties}

Previously, production of metal matrix SMA composites focused on powder metallurgy methodologies. In recent years, a number of novel processing approaches have been developed for MMCs, some of which have been adopted to NiTi-Al systems. For instance, Dixit et al. [112] manufactured NiTi particulate reinforced $\mathrm{Al}$ through friction stir processing and experimentally measured the residual stresses and strengths of composites. Although the friction stir processed composites did not exhibit significant 
Table 4: Metal Matrices with SMA Reinforcement.

\begin{tabular}{|c|c|c|c|}
\hline Matrix Material & Reinforcement Type & Reinforcement Material & References \\
\hline $\mathrm{Al}$ & Particulate & $\mathrm{NiTi}$ & {$[361-363,392,415,319]$} \\
\hline$"$ & Short Fiber & $"$ & {$[454,276]$} \\
\hline$"$ & " & NiTi (with SiC particles) & {$[84]$} \\
\hline$"$ & Long Fiber & NiTi & {$[8,147,341-343,185,186]$} \\
\hline$"$ & " & NiTi (with SiC particles) & [183] \\
\hline$"$ & Ribbon & NiTi & {$[161]$} \\
\hline $\mathrm{AlSi}$ & Short Fiber & $"$ & {$[299]$} \\
\hline $\mathrm{Cu}$ & Strips & $"$ & {$[263]$} \\
\hline $\operatorname{InSn}$ & Particulate & CuAlNi & {$[280-282,32]$} \\
\hline In & $"$ & $"$ & {$[391,281,282]$} \\
\hline $\mathrm{SnAg}$ & Long Fiber & $\mathrm{NiTi}$ & {$[446,447,102,103]$} \\
\hline $\mathrm{Fe}$ & Particulate & $"$ & {$[160]$} \\
\hline NbTi & Lamella & $"$ & {$[197,198,200,201]$} \\
\hline $\mathrm{Mg}$ & Particulate & $"$ & {$[127]$} \\
\hline$"$ & Long Fiber & $"$ & {$[461]$} \\
\hline$"$ & Interpenetrating & $"$ & {$[21]$} \\
\hline
\end{tabular}

residual stresses, subsequent processing through cold-rolling and annealing did produce residual stresses which resulted in substantial improvements in the yield strengths. Recent efforts by Ni et al. [319] have further developed this work for bulk processing by considering $5 \mathrm{~mm}$ thick specimens. Even with the larger materials, a homogeneous particle distribution was noted. Importantly, this route also inhibited the interfacial reaction which can degrade the material performance. This reaction layer has also been considered via separate approaches by $\mathrm{Hu}$ and colleagues [186] and Hahnlen and Dapino [161]. In the former case, the ability of pre-oxidation of the SMA fibers to limit diffusion between the $\mathrm{Al}$ and NiTi materials was studied. Through these investigations, it was shown that longer oxidation exposure lead to thicker reaction layers with weaker bonds and correspondingly lower elongation to failure and tensile strength [186]. The latter study used an ultrasonic additive manufacturing approach to join the materials at room temperature and avoid issues arising from pre-strain recovery and found the temperature at which the bond failed due to prestress [161].

Example stress-strain responses of SMA reinforced metallic matrices are presented Fig. 7a and b and example properties of various SMA-MMCs are listed in Table 5. The temperature dependence of the SMA constituent may be observed in the variation of the composite response in Fig. 7b.

Table 5: Room Temperature Properties of SMA Reinforcement Metal Matrix Composites.

\begin{tabular}{l|cccr}
\hline Property & \multicolumn{2}{|c}{ Al matrix w/ SiC particulate [183] } & Mg Matrix [21] \\
& $\mathrm{SiC}=0 \%$ & $\mathrm{SiC}=35 \%$ & & \\
\hline SMA vol\% & 20 & 20 & 10 & 30 \\
Elastic Modulus (GPa) & 38 & 77 & 44 & 45 \\
Yield Strength (MPa) & 64 & 136 & 87 & 113 \\
Elongation (\%) & 16.7 & 1.5 & 17.4 & 10.2 \\
\hline
\end{tabular}

\subsubsection{Applications}

Increased composite yield strength - One of the earliest motivations for using SMA reinforcement in a metal matrix composite was to increase the strength of the composite. As discussed by Furuya [147], the implementation of SMAs leads to two potential interesting mechanisms: (i) development of compressive residual or bias stresses to increase the apparent yield strength through the pre-strain assisted SME, and (ii) application of compressive stresses around a crack tip through the SME. The increase of apparent yield strength at higher temperatures was first shown experimentally in aluminum metal matrix composites reinforced with NiTi fibers [148,8,147] and particulates [361-363,392]. Recently, 


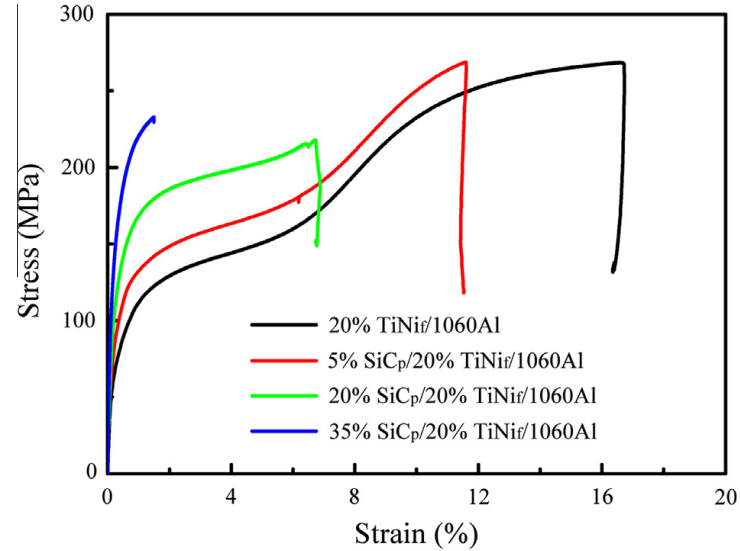

(a) Stress-strain response of an $\mathrm{Al}-\mathrm{SiC}$ matrix $(\mathrm{SiC}$ particle reinforcement in the $\mathrm{Al}$ matrix) composite containing 20 vol\% NiTi fibers at different $\mathrm{SiC}$ reinforcement concentrations. Reprinted from [183] with permission from Elsevier.

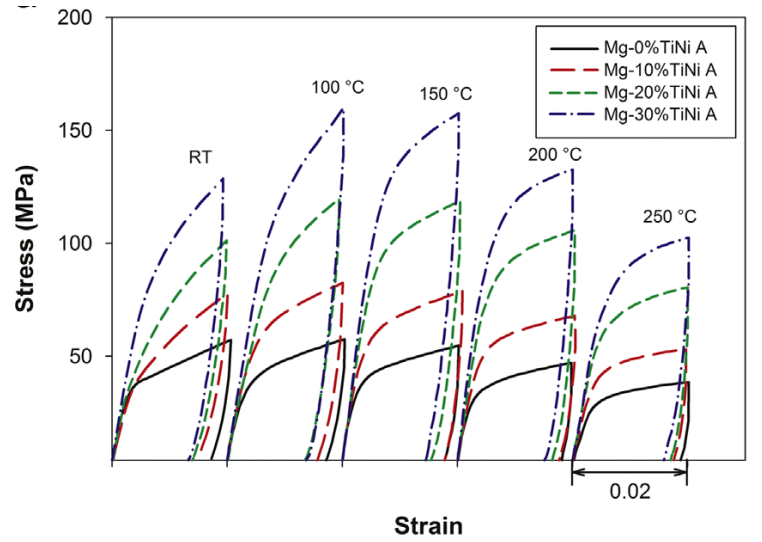

(b) Stress-strain responses of a Mg matrix, NiTi reinforced composite with different NiTi concentrations and at different temperatures. Reprinted from [21] with permission from Elsevier.

Fig. 7: Characteristic stress-strain response of SMA reinforced metal matrix composites: (a) Al and (b) Mg matrix systems.

similar investigations were performed in Al-NiTi composite systems. Short fiber reinforced composites were explored by Xie et al. [454] who showed an increase in composite strength through the SME. To aid in understanding of the role of the interface, TEM and energy dispersive spectroscopy (EDS) have been used to characterize the NiTi/Al interface both in the case of short [276] and long fibers [184]. The load transfer in Al-NiTi systems (30 vol\% NiTi fiber reinforcement) was also studied by prestraining the composite to different conditions and then thermally cycling the specimen and studying the developed dislocation structures [185]. In addition to the fracture properties, the fatigue properties of aluminum matrix composites reinforced by NiTi wires have also been explored [342,343,341]. A decrease in fatigue crack growth rate in composites exhibiting shape memory characteristics was noted by Park et al. [342]. Statistical S-N curves considering different volume fraction and reduction ratios were also determined through a series of experiments by Park et al. [341]. It was also shown that acoustic emission techniques may be used to analyze crack propagation in these composites [343].

The idea of strengthening metal matrices is also not restricted to aluminum systems. For instance, the utilization of NiTi as reinforcement has also been investigated for AlSi (33\% shear strength increase with $3 \%$ NiTi fibers) [299] and Mg [300,461,127,21] matrix systems. With respect to the latter case, Esen [127] considered different reinforcement content and demonstrated that although NiTi content increases yield strength and elongation, those benefits decrease with increasing NiTi content. Aydoğmus [21] similarly manufactured composites with different concentrations and determined the stress-strain response of these systems as a function of reinforcement content and temperature. From this study, it was observed that any NiTi reinforcement increases the strength and modulus of these materials and the apparent improvements actually grow with temperature up to $150^{\circ} \mathrm{C}$ (Fig. $7 \mathrm{~b}$ ). Interestingly, some studies have sought to combine both SMA and stiff, particulate reinforcement. Specifically, the possibility of developing Al matrix systems with NiTi-fiber and SiC particulate reinforcement has been explored considering both more mild (1.0 and $5.0 \mathrm{wt} \% \mathrm{NiTi}$ short fibers and $\mathrm{SiC}$ particulates, respectively) [84] and substantial (20 vol\% long NiTi fibers and up to 35\% SiC particulates) [183] reinforcements. An example microstructure of this latter combined approach is presented in Fig. 1d in which both the continual NiTi reinforcement and distributed SiC particles in the matrix can be observed. Both cases showed improvements in composite strength while the former also highlighted the beneficial impact of having fiber diameters closer to the grain size [84] to improve load transfer and avoid pull-out failures while the latter reported on the decrease in ductility (Fig. 7a) and damping due to the $\mathrm{SiC}$ content [183]. In a series of investigations, the effect of matrix constraint on martensitic transformation was studied in composites comprised of nano-crystalline NiTi grains within a $\mathrm{NbTi}$ 
matrix through thermal dilatometry $[197,198]$ and the effect of incomplete transformation $[200,201]$. The previous cases were all considered with materials with relatively stiff metal matrices. Strengthening through shape memory properties has also been pursued for much softer $\mathrm{Sn}-\mathrm{Ag}$ matrices for consideration as lead free solder. Wang et al. $[446,447]$ first looked at using NiTi reinforcement in a solder such that during initial heating, large thermal expansion mismatches detwinned the structure. Continued heating eventually induces reverse transformation and the corresponding recovered deformations placed a back-stress acting against creep [447]. Experiments through a thermomechanical cycle approximating service conditions demonstrated a significant reduction in creep rate (especially against a comparison $\mathrm{Cu}$ material) [446]. In additional experiments, Coughlin and coworkers $[102,103]$ extensively characterized the tension and fatigue characteristics properties finding $85 \%$ of permanent deformations of initial mechanical testing can be recovered through unloading (pseudoelastically) and indicating that fatigue is controlled by the fiber [102]. The interface that develops between the solder matrix and NiTi reinforcement was also investigated and its growth $\left(\right.$ at $\left.300^{\circ} \mathrm{C}\right)$ and elastic properties were quantified [103].

High-damping - Recent efforts have focused on the development of new, high-damping material composites. One such system under investigation is a CuAlNi SMA phase in different InSn matrices [32, $281,282,280,391]$. Additionally, new high-damping, high-strength NiTi-Mg composite has also been developed or characterized [260]. Novel CuAlMn SMA matrix composites with graphene particulates [439, 438] have also been manufactured. Explosive welding has been utilized to create CuAlMn-QBe2 composites [155]. The damping capacity of Al reinforced by NiTi particulates was also characterized through internal friction methodologies [415].

A series of efforts have also looked at using a soft, ferromagnetic Fe matrix with NiTi precipitates to create an effective MSMA. In this hybrid mechanism, large field gradients lead to the ferroelectric phase inducing load on the NiTi reinforcement which undergoes stress (rather than field) induced martensitic transformation [160]. A sample composite has been produced via spark-plasma sintering and characterized [160] while this concept has been used to create simple composite structures for use as a jet flow control [269] and inchworm [270]. NiTiCu-Pt bilayer composite has been created for use as a microactuator in MEMS devices [191,471]. By using a combination of focused ion beam (FIB) milling and chemical vapor deposition (CVD) techniques, bimorphs with a thickness of $140 \mathrm{~nm}$ have been shown to maintain their actuation capabilities. The ability of NiTi wire reinforcement to decrease the effective thermal expansion of the copper matrix has been studied for use in electronic applications [263].

\subsection{Ceramic Matrix Composites (CMCs)}

The use of SMA reinforcement in ceramic matrix systems has been primarily to improve the ductility or yield strength in brittle materials (like concrete) or enhanced multifunctionality. Typically, as shown in Fig. 5c-d, such composites may have limited actuation strains but potentially high actuation stresses. Examples of the different material systems considered are given in Table 6 and investigations into these composites are reviewed in this section.

\subsubsection{Processing Methodologies \& Properties}

As with many conventional ceramic systems, SMA reinforced ceramics are typically manufactured via powder methods and various sintering techniques. The utilization of such approaches, however, is often non-trivial, as Xiu and coworkers observed in attempting to produce a $\mathrm{Al}_{2} \mathrm{O}_{3}-\mathrm{Ti}(\mathrm{C}, \mathrm{N})$-NiTi. In this case, the sintering was performed above the melting temperature of the NiTi leading to its decomposition into other phases [456]. Extending the applicability of the aforementioned TiC inclusions, Strutt et al. $[408,409]$ investigated the use of self-propagating high-temperature synthesis (SHS) and quasi-isostatic pressing (QIP) to create $\mathrm{TiC}$ - NiTi composites using elemental powders while Yang and coworkers considered a molten-salt bath based approach [462]. In this former case, the authors noted the need to anticipate the development of $\mathrm{TiC}_{0.7}$ cermets in the composition of the additional powders to avoid the formation of detrimental $\mathrm{Ni}_{3}$ Ti phases [408]. The resultant microstructures were extensively characterized via electron microscopy to investigate the transformation mechanisms and martensite morphology [409]. Following on these efforts, new SMA-MAX phase ceramic composites 
Table 6: Ceramic Matrices with SMA Reinforcement.

\begin{tabular}{|c|c|c|c|}
\hline Matrix Material & Reinforcement Type & Reinforcement Material & References \\
\hline $\mathrm{TiC}$ & Particulate & $\mathrm{NiTi}$ & {$[408,409,462]$} \\
\hline $\mathrm{Ti}_{2} \mathrm{AlC}$ & $"$ & $"$ & {$[187]$} \\
\hline $\mathrm{Ti}_{3} \mathrm{SiC}_{2}$ & Interpenetrating & $"$ & {$[187,230,93]$} \\
\hline $\mathrm{Al}_{2} \mathrm{O}_{3}-\mathrm{Ti}(\mathrm{C}, \mathrm{N})$ & Particulate & $"$ & [456] \\
\hline Cement & Wire & NiTi & {$[219]$} \\
\hline$"$ & $"$ & $\mathrm{NiTiNb}$ & {$[219]$} \\
\hline$"$ & $"$ & $\mathrm{FeMnSi}$ & {$[274,396]$} \\
\hline Reinforced Concrete (RC) & Bar & NiTi/Glass Fiber Hybrid & {$[451,469,468]$} \\
\hline Engineered Cementitious Composite (ECC) & Cable & $\mathrm{NiTi}$ & [264] \\
\hline $\mathrm{PZT}$ & Lamina & $\mathrm{NiTi}$ & {$[311]$} \\
\hline$"$ & $"$ & NiMnCoSn & {$[90,91]$} \\
\hline$"$ & $"$ & $\mathrm{FePd}$ & {$[322]$} \\
\hline$"$ & $"$ & $\mathrm{NiFeGaCo}$ & {$[459]$} \\
\hline PMN-PT & $"$ & NiMnCoSn & {$[92]$} \\
\hline$"$ & $"$ & MnNiSn & [463] \\
\hline$"$ & $"$ & NiMnGa & {$[472]$} \\
\hline
\end{tabular}

(e.g., $\mathrm{NiTi}-\mathrm{Ti}_{2} \mathrm{AlC}, \mathrm{NiTi}-\mathrm{Ti}_{3} \mathrm{SiC}_{2}$ ) have been developed which take advantage of the unique inelastic kinking response of the MAX phases $[35,36]$. The manufacture of these new composites through spark plasma sintering is described by $\mathrm{Hu}$ et al. [187] who extensively considered the impact of sintering temperature and pressure on the formation of a reaction layer that decreased the transformable NiTi in the system. The compositional and elastic properties of the interface in the $\mathrm{NiTi}-\mathrm{Ti}_{3} \mathrm{SiC}_{2}$ system were also characterized [38,229] while the thermal properties at temperatures up to $1233 \mathrm{~K}$ [93] of the $\mathrm{NiTi}-\mathrm{Ti}_{3} \mathrm{SiC}_{2}$ composite have also been experimentally measured.

Unlike previous metal and polymer matrix systems, ceramic matrices are typically more than four times stiffer than NiTi and other SMA materials. Therefore, much of the load bearing capability is carried by matrix phase that also provides a constraint to the development of transformation strains in the system. Examples of the stress-strain response of a $\mathrm{TiC}_{0.7}-\mathrm{NiTi}$ with $30 \mathrm{vol} \% \mathrm{NiTi}$ reinforcement and the strain-temperature response of a $\mathrm{NiTi}-\mathrm{Ti}_{3} \mathrm{SiC}_{2}(50 \mathrm{vol} \% \mathrm{NiTi})$ composition are shown in Figs. $8 \mathrm{a}$ and b, respectively. Like the matrices themselves, SMA reinforced ceramic matrix materials typically have higher stiffnesses and strengths but more moderate strains. For instance, $\mathrm{TiC}_{0.7}$ cerments with $30 \%$ SMA reinforcement have an elastic modulus of $300 \mathrm{GPa}$ and a composite strength of 2800 $\mathrm{MPa}$. The strain to failure, however, is only $0.98 \%$.

\subsubsection{Applications}

Damage suppression - SMAs have long been proposed as reinforcement in concrete in order to develop desirable pre-stresses thereby improving the yield strength of the material. Most efforts have focused on NiTi reinforcement although new SMA reinforcements have been considered. In particular, the use of an iron based FeMnSi alloy has been investigated $[274,396]$ and NiTi reinforced polymer matrix composite (PC-SMAs) [451,469,468] have all been explored. This latter reinforcement has been incorporated into small scale concrete structures and demonstrated the enhancement of dissipation, ductility, and crack-closing capabilities through three point bending tests [470]. A recent, extensive experimental investigation has also demonstrated similar gains by incorporating SMA reinforcement into engineered cementitious composites (SMA-ECC) by Li et al. [264]. Through DIC investigations, they demonstrated a recover in distributed damage by using SMA reinforcement (see Fig. 2c) indicating a significant selfrepair capability. Like previous materials, the bonding between the SMA reinforcement and cement mortar poses a critical issue in terms of load transfer to take advantage of these behaviors and the corresponding composite strength. Therefore, Kim and colleagues [218,219] performed a series of tests looking at ways to improve the bond strength between the SMA wires and surrounding cement. To this end, it was shown that processing via cold-drawing (and the corresponding increase in stiffness) and heat treatments increase the bond strength [218] while pre-deforming the reinforcement into dog-boned or end-deformed configurations will also yield gains the bond performance [219]. 


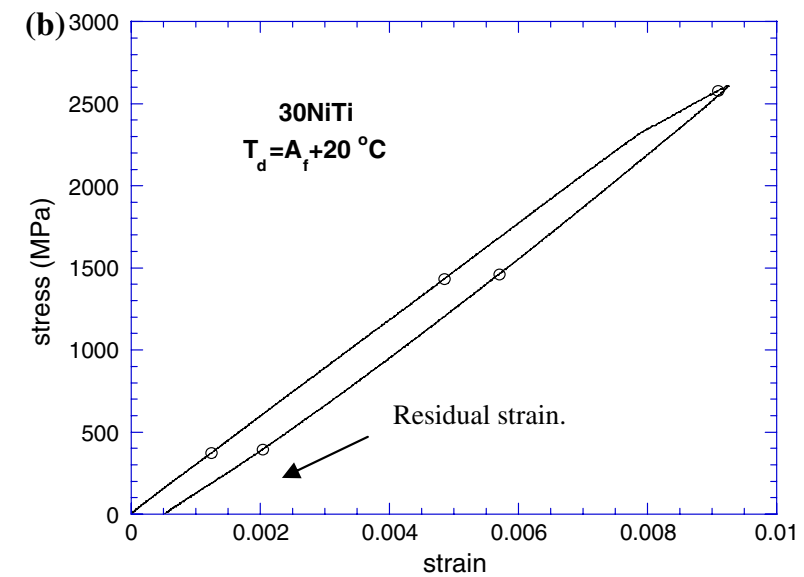

(a) Stress-strain response of a $\mathrm{TiC}_{0.7}-\mathrm{NiTi}$ (30 vol\% $\mathrm{NiTi}$ ) composite subjected to a quasi-static compressive loading at $T=A_{f}+10^{\circ} \mathrm{C}$. Reprinted from [408] with permission from Springer.

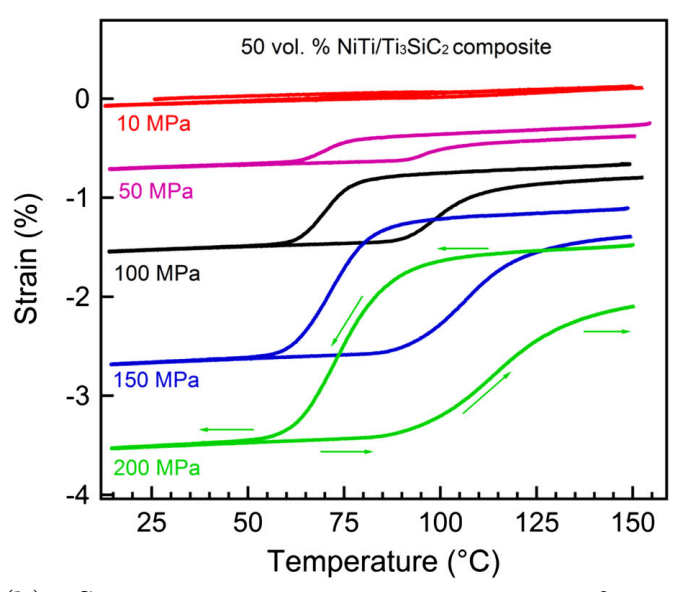

(b) Strain-temperature response of a $\mathrm{NiTi}-\mathrm{Ti}_{3} \mathrm{SiC}_{2} \quad\left(\begin{array}{llll}50 & \text { vol\% } & \mathrm{NiTi}\end{array}\right)$ composite system under constant applied compressive stresses of the indicated magnitude. Reprinted from [230] with permission from Springer.

Fig. 8: Characteristic responses of SMA reinforced ceramic matrix systems: (a) stress-strain and (b) strain-temperature responses of different composite systems.

High-damping - The thermomechanical response and transformation characteristics of $\mathrm{NiTi}-\mathrm{Ti}_{3} \mathrm{SiC}_{2}$ have been characterized by Kothalkar et al. [230] who also note the improved mechanical damping of these unique material systems. This response is associated with the combination of inelastic mechanisms and these composites have been shown to exhibit an effective mechanical damping over a mechanical loading-unloading cycle even greater than the separate constituents. Constant stress cooling heating cycles have been shown to increase the dissipative of these composites which is associated with internal stress generation during these thermomechanical paths. These residual stresses also lead to the development of an effective two-way shape memory effect (TWSME) behavior in the composite not otherwise observed [230].

Improved multifunctionality - Novel ceramic matrix composites have also been developed in which piezoelectric constituents are incorporated to produce additional multifunctional couplings - notably the magnetoeletric (ME) and thermal-electrical-elastic effects. With respect to the former, a number of laminates with MSMA and piezoelectric layers have been produced leading to an enhanced ME effect. The observed couplings are even stronger than that observed in magnetostrictive (e.g, Terfanol-D) based composites. Specifically, in a series of efforts, Chen et al. [90-92] investigated the possibility of using NiMnCoSn as the MSMA phase to produce both the direct magnetoelectric (DME) and converse magnetoelectric (CME) effects. The first, DME, is the result of applied magnetic fields inducing MFIS in the MSMA with the resultant deformations mechanically loading the piezoelectric phase to produce a voltage. On the other hand, an electric field being applied to the piezoelectric material leading to stress induced martensitic transformation (and the corresponding large change in magnetization) is referred to as the CME. In both the DME [91] and CME cases, large coupling coefficients were measured and in the CME case the values reported were much larger than those of most other composites [90]. Zeng et al. [472] studied the effect of martensitic transformation on the CME of a NiMnGa/PMN-PT structure and noticed a maximum induced magnetization during phase transformation. The coupling between the hall resistivity and stress-induced martensite was also characterized [92]. The DME response of FePd [322] and CME of NiFeGaCo [459] were also investigated and in the latter case it was reported that an electric field applied to the piezoelectric layer could control the corresponding CME. Furthermore, in the case of MnNiSn/PMN-PT laminate, it was noted that the electric bias had a strong effect on the CME was observed and, importantly, magnetization reversal was observed without a bias field [463]. Similar effects have been explored for power generation and energy harvesting. To this end, 
the combination of thermally induced transformation piezoelectric effect in an SMA and PZT layer, respectively, was investigated both experimentally [311] and numerically [311,248].

\subsection{SMA Matrix Materials}

In the previous three sections, the SMA phase was always taken to be the reinforcement. A number of material systems, however, have been developed in which the SMA component is the matrix. Such cases have focused primarily on either (i) reinforcing the SMA matrix material to increase effective yield strength or porous SMAs for use in low-stiffness cases with low densities (as seen in Fig. 5d). These efforts are presented in this section and examples of the different material systems explored are presented in Table 9 .

Table 7: Composites using SMA as the Matrix Phase.

\begin{tabular}{|c|c|c|c|}
\hline Matrix Material & Reinforcement Type & Reinforcement Material & References \\
\hline $\mathrm{NiTi}$ & Particulate & $\alpha-\mathrm{Al}_{2} \mathrm{O}_{3}$ & {$[133]$} \\
\hline$"$ & $"$ & $\mathrm{Mg}$ & {$[260]$} \\
\hline$"$ & $"$ & W & {$[441]$} \\
\hline$"$ & $"$ & $\mathrm{TiC}$ & {$[293,146,145,119,202,429-432]$} \\
\hline$"$ & $"$ & $\mathrm{SiC}$ & [199] \\
\hline$"$ & $"$ & CNTs & {$[134,246]$} \\
\hline$"$ & Nanowires & $\mathrm{Nb}$ & {$[166,167,277-279]$} \\
\hline$"$ & Bilayer & $\mathrm{NiTiCu}$ & [108] \\
\hline$"$ & Coaxial Wire & $\mathrm{Fe}$ & [168] \\
\hline $\mathrm{NiTiCu}$ & Bilayer & $\mathrm{Pt}$ & {$[471]$} \\
\hline $\mathrm{CuAlMn}$ & Particulate & Graphite & {$[438,439]$} \\
\hline$"$ & $"$ & Polystyrene & {$[437]$} \\
\hline NiMnGa & Fill & Polyurethane & {$[384]$} \\
\hline$"$ & Bilayer & PVDF & {$[473]$} \\
\hline \multirow[t]{3}{*}{$\mathrm{NiTi}$} & Porous & - & {$[194,257-259,265,266,483,26,27]$} \\
\hline & & & $\&[30,31,455,182,309,275,50,51]$ \\
\hline & & & $\&[24,22,23,315,316,227,476,54,66]$ \\
\hline $\mathrm{NiTiCu}$ & $"$ & - & {$[467]$} \\
\hline $\mathrm{CuAlMn}$ & $"$ & - & {$[436,435,440]$} \\
\hline $\mathrm{CuZnAl}$ & $"$ & - & {$[77,52,53,10]$} \\
\hline $\mathrm{NiTiNb}$ & $"$ & - & {$[262]$} \\
\hline $\mathrm{NiMnGa}$ & $"$ & - & {$[64,96,95,477,452,484]$} \\
\hline NiCoMnSn & $"$ & - & [301] \\
\hline
\end{tabular}

\subsubsection{Processing Methodologies \& Properties}

Porous - Porous NiTi was first explored for use in biomedical applications in the 80's and early 90's in Russia. A summary of these efforts spanning initial production methods and characterization of pseudoelastic and shape memory responses were reported by Itin et al. [194]. In general, like other porous metals, different powder metallurgical techniques (a number of which are reviewed by Ryan and colleagues [386]) are used to produce porous SMAs. With SMAs in general, and NiTi (by far the most common porous SMA) in particular, a number of additional processing complexities are noted. First, slight compositional variation can have a pronounced effect on transformation characteristics necessitating methodologies that have tight diffusional control [413,324]. Secondly, NiTi has a high melting temperature and is chemically reactive with many crucible materials further complicating the manufacturability. Early investigations focused using elemental powders with conventional sintering [257], self-propogating high-temperature synthesis (SHS) [259,258,265,266], and hot isostatic pressing (HIP) [234]. Through such investigations specimens with a wide range of porosities (up to $64 \%$ [265]) were produced. These methodologies, however, provided limited control of pore distribution 
and shape and in the SHS case pore directionality and anisotropy was noted under some processing conditions [265]. Additionally, the usage of elemental powders enabled the creation of potentially substantial quantities of various non-transforming, intermetallic phases via the sintering [257], SHS [258], and HIP [234] methods. With the former method, $\mathrm{Li}$ and coworkers [257] also considered using $\mathrm{TiH}_{2}$ powders in lieu of $\mathrm{Ti}$ and noted a more homogeneous, decreased pore size in the material specimens.

In recent years, a number of advances have been made to address these issues and improve shape control and distribution of the porosity. Specifically, microwave sintering [458], metal injection molding (MIM) [193], and a number of spaceholder $[478,455,482,261,310,476,464]$ techniques have all been developed to address porosity control. With respect to the latter category, $\mathrm{NaCl}[476,482,464], \mathrm{Mg}[310]$, $\mathrm{NH}_{4} \mathrm{HCO}_{3}[478,455]$, and $\mathrm{CO}\left(\mathrm{NH}_{2}\right)_{2}[261]$ have been utilized. By using $\mathrm{NaCl}$, for instance, specimens with porosity reaching $90 \%$ [482], a bimodal pore distribution (by using two sizes of placeholder thereby creating micro and macropores increasing the damping properties) [476], and gradient porosity [478] have been manufactured. Yen and coworkers used $\mathrm{NaCl}$ as spaceholder to produce specimens with a range of porosity $(30-80 \%)$ and reported $M_{s}$ and transformation enthalpies that were independent of porosity. High-porosity specimens (up to $87 \%$ ) were also manufactured using a $\mathrm{NH}_{4} \mathrm{HCO}_{3}$ spaceholder [455]. Exploiting the elemental powder basis, NiTiNb (with up to $10 \mathrm{at} \% \mathrm{Nb}$ ) specimens have been created [262] while Chen et al. used $\mathrm{TiH}_{2}$ powders to produce specimens with higher porosity, fracture toughness, and pseudoelastic strain versus comparable materials made with $\mathrm{Ti}$ [88]. Hosseini and colleagues [182] considered the impact of post manufacture (via sintering) heat-treatments via dilatometry and noted that aging increases austenitic transformation temperatures and shape-recovery character.

To improve compositional control and avoid the creation of detrimental intermetallic phases, prealloyed NiTi powders have been extensively utilized. Techniques including solid-state foaming via creep expansion [323] and a variety of spaceholder techniques have been developed using these powders. Specifically, Aydoğmus and coworkers [24,22] investigated using $\mathrm{Mg}$ as a spaceholder and studied the effect of aging [24] as well as demonstrating the possibility of using $\mathrm{Mg}$ both as a spaceholder and a reductant to avoid any second phase intermetallic formation [22]. Bansiddhi and Dunand, on the other hand, utilized $\mathrm{NaF}[26]$ and $\mathrm{NaCl}[27]$ as spaceholders with the latter effort producing specimens with a greater than $1000 \mathrm{MPa}$ failure stress and $4 \%$ strain recovery from an $8 \%$ applied strain [27]. To improve on these results, the same investigators added small amounts of Nb powders to create a transient eutectic liquid phase which, through capillary forces, wick into microcavities and improve densification [28]. Subsequent efforts led to a two-step process in which elemental Ni could be used to create a shape-memory Ni-rich NiTi composition [30]. Niobium short fibers were also used in lieu of the $\mathrm{NaCl}$ spaceholder to simplify the process and produce a porous specimen with elongated pores [29]. To get even finer control of the porosity shape, 2D meshes of steel wire were inserted into NiTi to serve as spaceholders (later removed electrochemically) and create controllable microchannels [316] (an example of this microstructure is given in Fig. If in which the controlled porosity is readily apparent). Bewerse et al. [54] extended this concept to create three-dimensional networks by first sintering the steel meshes and then using the Nb-based transient liquid processing technique described earlier to create the porous materials. New near net-shape manufacturing methodologies have also been employed to produce porous specimens. Specifically, laser engineered net shaping (LENS) [50,51] and selective laser melting (SLM) [66] have both been used. Specifically, the latter method was utilized in the development of materials tailored to expected mechanical stimuli of surrounding bones and tissue ingrowths while optimizing the fatigue resistance [66].

Although most investigations have centered on NiTi, a number of other porous SMAs have also been created. For instance, Young et al. [467] created a $60 \%$ porous $\mathrm{NiTiCu}$ specimen using a $\mathrm{SrF}_{2}$ spaceholoer and liquid melt replication technique which exhibited $4 \%$ recoverable strain. For damping applications, different $\mathrm{Cu}$-based and MSMAs have also been developed. With respect to the former, CuAlMn specimens have been manufactured via water-soluble salt [436] and $\mathrm{NaCl}$ [435] spaceholder sintering-dissolution methods for potential as high-damping materials. Porous CuZnAl have also been developed using a molten metal infiltration technique [77]. The effect of pore size produced via this method (controlled by $\mathrm{SiO}_{2}$ spaceholder bead diameter) on final response was studied and it was shown that smaller pore sizes can lead to larger pseudoelastic and shape memory recovery at large strains [10]. Inducing porosity (potentially substantial quantities) in polycrystal MSMAs has been proposed as a way to increase MFIS related to domain reorientation by decreasing constraints on twin boundary motion. To this end, Boonyongmaneerat et al. [64] used replication casting to create a porous NiMnGa 


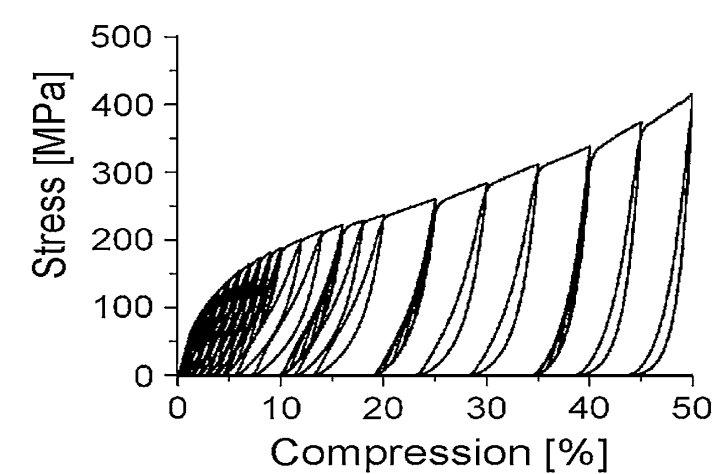

(a) Stress-strain response of a $51 \%$ porous NiTi specimen subjected to sequential compressive mechanical cycles at $T=A_{f}+11^{\circ} \mathrm{C}$. Reprinted from [70] with permission from Springer.

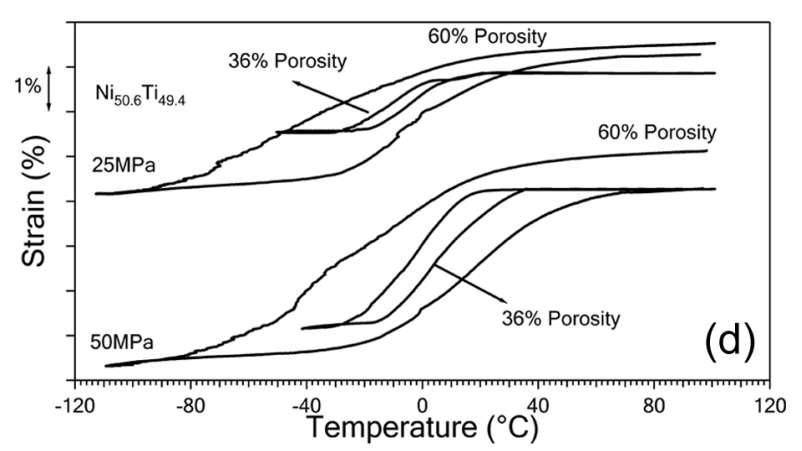

(b) Strain-temperature response of porous NiTi specimens with $36 \%$ and $60 \%$ porosity through coolingheating cycles under constant applied compressive loadings of 25 and $50 \mathrm{MPa}$. Reprinted from [227] with permission from SAGE (C)2013 Kockar et al.

Fig. 9: Characteristic responses of SMA matrix material systems: (a) stress-strain and (b) straintemperature responses of porous NiTi.

specimen with $0.115 \%$ MFIS (more than an order of magnitude higher than a fine-grained, dense specimen). Chmielus and coworkers [95] used neutron diffraction to experimentally demonstrate that the MFIS was a result of pores reducing internal constraints. Subsequent efforts sought to build upon this idea by creating porous specimens with bimodal pore architectures $[477,96]$. For instance, two different sized $\mathrm{NaAlO}_{2}$ spaceholder powders were used to produce the bimodal pores and increase the MFIS by roughly a sixth versus a single sized pore material [477] and thermal cycling between the phases produced an exceedingly high MFIS of $8.7 \%$ [96]. Porous materials taking advantage of magnetic field induced phase transformation have also been developed. Specifically, Monroe et al. [301] used solid state replication techniques to produce a NiCoMnSn specimen that underwent such a transformation.

Porous SMAs, especially foams with higher porosity, are typically considered for their low-stiffness, high-strength and deformation characteristics. Examples of the stress-strain and strain temperature responses are shown in Fig. 9a and b, respectively. The mechanical properties of NiTi foams was extensively investigated and characterized by Bram et al. [70] who considered a $51 \%$ porous specimen produced via metal injection molding and some representative properties of different porous SMAs are given in Table 8.

Table 8: Properties of Porous SMAs.

\begin{tabular}{l|ccc}
\hline Property & NiTi[70] & NiTi[315] & CuZnAl [53] \\
\hline Porosity (vol \%) & 51 & 20 & 75 \\
Elastic Modulus (GPa) & 6 & $6-12$ & 3 \\
Max Recoverable Strain (\%) & 6.5 & 6.5 & 3.4 \\
\hline
\end{tabular}

Dense SMA Matrix Materials - The effect of small concentrations of various reinforcement on a NiTi matrix has been studied. Specifically, low concentration ceramic inclusions of SiC particles (3 and $5 \mathrm{wt} \%)$ [199] and $\alpha-\mathrm{Al}_{2} \mathrm{O}_{3}(6 \mathrm{wt} \%)$ [133] have been incorporated with NiTi powders and the resultant $\mathrm{NiTi}$ system was shown, in the former case, to have increased strength while maintaining the shape memory characteristics of the matrix [199]. Interestingly, in the latter $\alpha-\mathrm{Al}_{2} \mathrm{O}_{3}$ specimen, a pronounced increase in transformation temperatures (with the smallest jump being $71^{\circ} \mathrm{C}$ ) and order of magnitude reduction in transformation enthalpy - likely associated with thermal stresses - were reported [133]. Higher concentrations (up to 20\%) of inclusions have also been extensively studied through the effect of TiC particles $[293,146,145,119,202,429,430,432,431]$. The utilization of unreacted CNTs (0.5 wt\%) [246] and graphitized multi-walled CNTs (5 vol\%) [134] as a reinforcing agent was also 
studied. In the unreacted CNT case an increase in modulus, yield, and ultimate strength was reported with some degradation of the shape memory characteristics [246] while the multi-walled CNTs showed similar improvements although much of these gains were lost at higher sintering temperatures leading to increased formation of $\mathrm{TiC}$ and interfacial reactions [134].

At a larger scale, NiTi tubes have also been joined with a soft Fe core to create a coaxial composite wire that exhibit high strain recovery and damping potential [168]. Enhanced multifunctionality NiTiNiTiCu SMAs has also been produced by locally heating Ti and $\mathrm{Cu}$ films between two NiTi sheets [108]. Recently, W-NiTi composites have also been improved for biomedical applications in which the W addition increases the radiopacity while maintaining the functional SMA characteristics [441]. All such investigations have produced SMA matrix material with a wide variety of properties. Some reference properties are presented in Table 9.

Table 9: Properties of Dense NiTi Matrix Materials.

\begin{tabular}{l|ccc}
\hline Property & \multicolumn{2}{|c}{ CNT } & Nb nanowires [166,167] \\
\hline Reinforcement (vol\%) & 0.5 & 1 & 25 \\
Elastic Modulus (GPa) & 79 & 77 & 28 \\
Recoverable Strain (\%) & 3.3 & 3.6 & $4-7$ \\
Strength (MPa) & 192 & 177 & 1650 \\
\hline
\end{tabular}

\subsubsection{Applications}

Biomedical Implants - Porous SMAs have excellent potential for biomedical applications due to the biocompatability of NiTi (and some ternary alloys like NiTiNb), possibility of bone ingrowth, and stiffness tailoring to minimize stress shielding effects. The possibilities of such implants has been reviewed by Elahinia et al. [123] and Bansiddhi et al. [31] with the latter also elaborating on biocompatability issues. Liu and coworkers [275], in an extensive series of investigations, studied the biomechanics of porous NiTi. Specifically, they investigated the osteointegration and pull-out loads of dense and porous NiTi and Ti (after implantation into rabbits) and noted the improved performance of porous NiTi [275]. For use in these applications, their thermomechanical response (especially compressive [266, 483]) has been extensively studied. Nemat-Nasser et al. [312] investigated the quasi-static and dynamic mechanical response of lower porosity specimens $(12 \%)$ and reported a strain-rate sensitivity comparable to the bulk material. Neurohr and Dunand [315] extensively characterized the mechanical response of NiTi with 2D microchannel networks and quantified the contributions of different strains (e.g., elastic, pseudoelastic, plastic, and recoverable) to the total strains over multiple cycles. Importantly, they noted more than $93 \%$ of seemingly permanent strain upon unloading from a $6 \%$ applied load may be recovered through heating and cyclic stability is reached after only a couple of mechanical cycles. Similar strain recovery was also noted for metal injection molding specimens by Ismail et al. [192]. The impact of training through repeated mechanical cycles and the stress level they were conducted at was considered by İpek Nakaş and colleagues [310] while Aydoğmus and Bor [23] showed that pre-straining and cyclic behaviors decreased the ductility. Fatigue characteristics of porous NiTi have also been extensively characterized [309,50,51]. İpek Nakaş et al. [309] noted three stages in the fatigue response: (i) rapid increase of initial inelastic strain due to localized plastic deformation, (ii) minimal, stabilized permanent strain accumulation associated with microcrack formation, and (iii) ultimate failure when the microcracks combine. Both the bending and compression fatigue properties of low-porosity specimens were measured by Bernard and coworkers [50,51]. With respect to specimens produced through microwave sintering, $\mathrm{Xu}$ and coworkers [457] measured the stiffness, compressive strength, and bending strength as a function of porosity. The isobaric response of NiTi foams with different porosities has also been investigated by Kockar et al. [227]. The resulting recoverable and irrecoverable strains generated through cooling-heating cycles at different applied, constant compressive stresses was measured. For instance, a $36 \%$ porous specimen was observed to have $4.5 \%$ recoverable strain and minimal irrecoverable strain at modest stress levels (100 MPa) [227]. Bertolino et al. [52,53] has also performed extensive characterization on CuZnAl foams. Although under small stresses, a clear pseudoelasticity 
was observed (recovery of about $95 \%$ of a $4 \%$ applied strain upon unloading [52]) with repeatability demonstrated up to 1000 cycles. The twinning stress, modulus, and damping of these materials as a function of porosity was also reported [53].

High-damping - The damping properties of various porous SMAs have also been investigated. For instance, Zhang and coworkers used DMA characterization to demonstrate the high-damping capacity of bimodal NiTi [476]. More extensive characterization was performed by Wang et al. [436] on the CuAlMn system via internal friction techniques. Specifically, increased damping was noted due to larger pore volume fractions or smaller diameters associated with additional local stress concentrations and related dislocation generation as well as refinement of the martensitic structure. Similar increases were also noted by adding an additional aging step in the manufacturing process [440]. A far greater enhancement was noted by filling the porosity with viscoelastic polystyrene arising from the additional dissipative material volume and additional interfaces [437]. A similar filling concept was also used by Romberg et al. [384] to increase the MFIS of NiMnGa. Specifically, controlled cracking was induced in bulk polycrystalline NiMnGa and later filled by polyurethane producing a polymer-NiMnGa composite with MFIS of more than $1 \%$.

Improved yield strength - The possibilities of the NiTiNb system was further explored by Hao et al. [166] who utilized severe plastic deformation to change Nb lamellae into Nb nanowires. The resulting $\mathrm{Nb}$ nanowire reinforced NiTi composite exhibited very high strengths $(1.65 \mathrm{GPa})$ while maintaining low elastic moduli (26 GPa) and high elastic strains (>6\%) [166]. Subsequent studies also demonstrated this novel composite had a narrow hysteresis with high energy efficiency and storage capabilities [167] while also investigating the role of annealing and pre-straining on the temperature dependent response [278] and studying the role of plastic deformation in the nanowire [279] and internal stress fields [277] on the composite response.

In addition to the previous cases taking advantage of SMA properties, novel architectures have also been created to enhance the functionality of porous media. For instance, Coppola et al. [100] fabricated a hybrid PMC/bulk NiTi bilayer in which low volume fractions $(<1 \%)$ of microchannels were incorporated in both layers via sacrificial component vaporization techniques. Subsequent tests showed more than an order of magnitude increase in maximum applied heat fluxes applied to the NiTi in which the PMC layer stayed below its glass transition temperature.

\section{Modeling Methodologies and Approaches}

Efforts to model and analyze the behaviors of SMA composites are reviewed in this section. First, modeling approaches to describe the response of bulk SMAs are briefly reviewed in Section 4.1. Micromechanical modeling approaches to explore the effective response of the composite and investigate the impact of the microstructure are discussed in Section 4.2. Finally, approaches to consider the response of structures incorporating SMA composites are summarized in Section 4.3.

\subsection{Bulk SMA Modeling}

Most efforts to model the response of bulk SMAs and their unique thermomechanical response fall into one of two categories: micromechanical or phenomenological. Both avenues have been extensively investigated over the previous decades and the reader is referred to the reviews of Patoor et al. [345] and Lagoudas et al. [238] for detailed discussions on micromechanical and phenomenological modeling methodologies for single- and polycrystals, respectively. Modeling at the single crystal level deals with the kinematics of phase transformation and the development of the free energy and dissipation potentials to derive constitutive equations with the aid of micromechanics (e.g., [344,188, 254]). Polycrystalline modeling, on the other hand, is approached via micromechanical averaging of the single crystal responses or phenomenological methods which postulate free energy functions for the macroscopic response via the appropriate selection of internal variables (e.g., $[19,334,86,233])$. The former approaches are typically prohibitively expensive for the analysis of large volumes or engineering structures so the latter are more closely aligned with the needs of SMA composite modeling.

Therefore, for use in subsequent composite discussions, the phenomenological modeling of polycrystalline SMAs based on the legacy of classical plasticity is briefly discussed. Generally speaking, such 


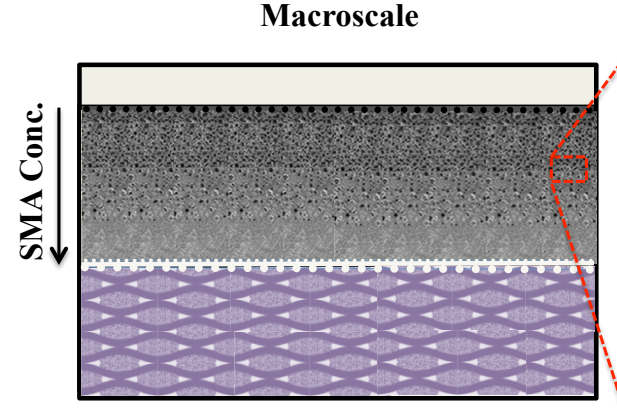

(a)

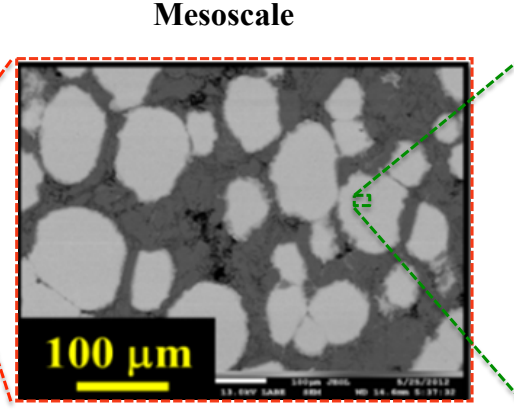

(b)

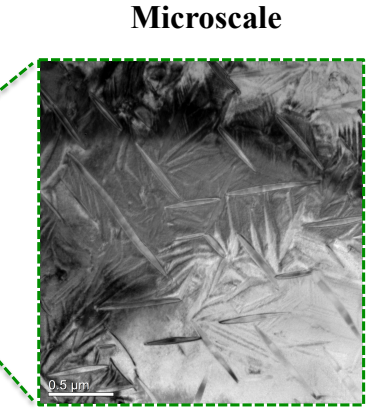

(c)

Fig. 10: Multiple scales of interest in the analysis of SMA composite materials: (a) Macroscale A functionally graded, hybrid composite for extreme environments ${ }^{2}$; (b) Mesoscale - A NiTi (white gray) $\mathrm{Ti}_{2} \mathrm{AlC}$ (darker gray) with heterogeneous microstructure, porosity (black), and reaction layers (Reprinted from [187] with permission from Elsevier); and (c) Microscale - Martensitic twins and accompanying nanoprecipitates of a $\mathrm{NiTi}^{3}$.

approaches utilize an additive decomposition of the infinitesimal strain increment with contributions from the elastic, thermal, and inelastic transformation strains. Although all three portions may change with phase, the latter tensor reflects the generation of macroscopic transformation strain associated with the formation of detwinned martensite. The prediction of the progression of the transformation strain tensor represents the core of phenomenological SMA modeling and the associated approaches borrow from classical metal plasticity. Specifically, internal variable(s) are introduced and evolution equations relating changes in the transformation strain increment and associated internal variables postulated along with corresponding yield functions. By using different forms of these relations, various elements of the phenomenology of the SMA response may be emphasized. To this end, models have been developed capturing the actuation [233], detwinning and reorientation [19,334,86], minor loop formation $[395,86]$, and coupling with additional inelastic mechanisms like plasticity $[395,173]$ and viscoplasticity $[171,85]$. The form of the corresponding yield functions has also been extensively been investigated [367] enabling the consideration of additional features like tension-compression asymmetry $[19,67,86,333,389]$. Many different forms of such models have been used in what follows to explore the impact of such responses on the underlying micromechanics of corresponding composites and will be discussed accordingly.

\subsection{Micromechanics of SMA composites}

The modeling and analysis of SMA composites, whether for the response of a structure or investigation of the underlying micromechanics, is an inherently multiscale task. Any such efforts seek to capture the response of the microstructural evolution in the SMA phase in conjunction with the heterogeneity at the composite meso- and macroscales. These various elements are schematically represented in Fig. 10 which identifies the macro-, meso-, and microscales in the context of a recently investigated functionally graded hybrid composite concept for extreme environments. At the macroscale (Fig. 10a), the hybrid composite is comprised of a thermal barrier ceramic layer [37], a functionally graded SMA-MAX phase metal-ceramic composite [187,230], and an actively-cooled, microvascular PMC [405]. Individually, each material introduces its own length scale(s) (e.g., relative thicknesses, concentration gradient) and the application of any thermomechanical loading conditions introduce additional structural considerations. The local microstructure of each phase becomes important at the mesoscale (Fig. 10b) where length scales associated with the reinforcement phase distribution, orientation, and sizes must be introduced in the analysis. In the specific cases presented, additional phases in the form of porosity and a reaction layer/interphase [187] are also evident and need to be accounted for. The presence of the SMA also introduces lower, microscale length scales associated with the size of martensitic variants and precipitates - the ratio of these two has been shown to strongly impact the characteristics of SMAs [129-131]. 


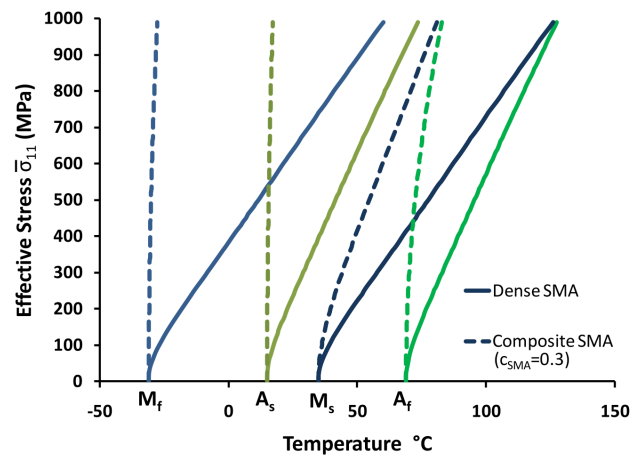

(a) Effective phase diagram of a stiff, ceramic composite reinforced with $30 \%$ SMA spherical reinforcement compared with the bulk SMA behavior. Substantial differences in the effective transformation temperatures may be noted and need to be accounted for in composite design. (CIOP Publishing. Reproduced from [252] by permission of IOP Publishing. All rights reserved.

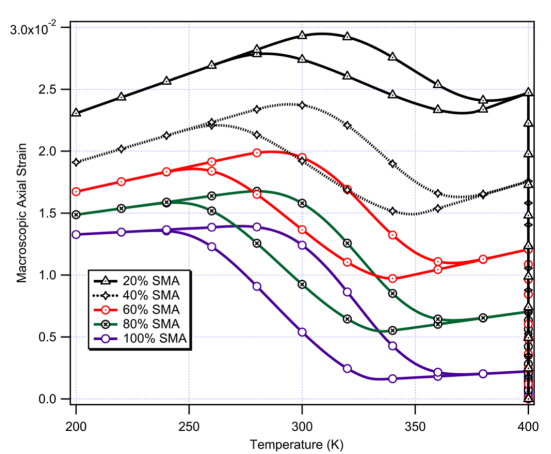

(b) Isobaric, strain-temperature response of a multilayered NiTi/epoxy composite isobaric thermal cycle with different concentrations of NiTi reinforcement under an applied $200 \mathrm{MPa}$ (normal to the laminate) loading. (C)IOP Publishing. Reproduced from [82] by permission of IOP Publishing. All rights reserved.

Fig. 11: Examples of bulk and composite SMA behaviors highlighting the impact of the additional behaviors on the effective transformation responses.

Considering all of these different heterogeneities, a key challenge in the modeling and analysis of SMA composites is how to bring the lower scale analysis up (or in some cases the inverse). Typically, the microscale features are accounted for in terms of homogenized constitutive laws of the SMA behavior and micromechanical analysis is performed at that scale (e.g., $[39,104])$. Substantial effort, however, has been expended to analyze the higher scales.

The micromechanical methods utilized in the literature of SMA composites fall into two broad categories - mean-field approaches and full-field approaches. In essence, all efforts in this category need three key components: (i) constitutive models of each phase (ii) identification of localization tensors which relate the global and local responses and (iii) techniques to transition and bridge the different scales. The former category is covered by approaches in Section 4.1 and will not be discussed in detail here while the micromechanical approaches discussed in the following tackle the latter two components. Methodologies considering only the phase-averaged response of the various constituents are defined to be mean-field approaches while full-field methods are those in which the position dependent field values are determined and then averaged for the effective, macroscopic response [244]. The former approaches typically leverage the seminal result of Eshelby [128] and his Equivalent Inclusion Method $[128,305,371]$ to account for the presence of multiple inhomogeneities while the latter category utilize numerical techniques like the finite element method (FEM) to solve representative computational domains - repeating unit cells (UC) for periodic materials and representative volume elements (RVEs) for statistically homogeneous materials [354]. These methodologies have been extensively developed for heterogenous materials with different nonlinear constitutive responses [354]. Notably, comparisons between different mean-field and full-field approaches and the relative benefits considering elastic-plastic [156,78,398,352,114,244], viscoplastic [353,113], and damage [79] responses have all been considered and may be found in the literature. In the following, the specific case of SMA constituents and the ensuing complexities are considered.

Shape memory alloys introduce additional constitutive complexities not observed in conventional elastic or elastic-plastic materials that must be accounted for in the micromechanics-based theories and methodologies for modeling the response of SMA composites: (i) phase transformation properties of the composites, such as bounded and recoverable inelastic deformations and phase transition tem-

${ }^{2}$ Courtesy of the various PIs of the AFOSR MURI (award number FA9550-09-1-0686) investigating the fabrication, synthesis and characterization of the depicted composite.

${ }^{3}$ Courtesy of Prof. Karaman's and his group (Department of Materials Science, Texas A\&M University). 
peratures, (ii) strong thermomechanical coupling, (iii) changes in thermoelastic properties, and (iv) Transformation-Induced Plasticity (TRIP). Many of these constitutive complexities transfer to the effective SMA composite response and need to be determined at the macroscopic level. Examples of such effective transformation characteristics are given in Fig. 11 in which the effective phase diagram (Fig. 11a) and effective strain-temperature response through a cooling-heating cycle under constant load (Fig. 11b) are presented. As can be observed in Fig. 11a, the temperature at which a composite transforms (at a given applied macroscopic load) may shift substantially due to the presence of a matrix/reinforcement phase. In terms of the actuation response, in Fig. 11b, it is noted that as the SMA reinforcement decreases, the transformation temperatures increase and the observable recoverable transformation strain and hysteresis are lowered. Determination of these parameters are essential for the design of SMA composite material systems and appropriate methodologies for determining them must be developed. Additionally, as highlighted by the responses presented in Fig. 11b, thermally induced deformations are often essential for SMA composites unlike most elastic-plastic materials in which only the mechanical response is analyzed.

The micromechanics of heterogeneous materials essentially reduces to two problems - localization and homogenization. The former dealing with mapping effective properties and state to local inhomogeneities and the latter associated with determining effective responses knowing local ones. Both are accomplished via the determination of the average strain and stress concentration tensors of the $i$-th phase, $\mathbf{A}^{(i)}$ and $\mathbf{B}^{(i)}$, respectively. Specifically, the strain concentration tensors relates the average strain of the $i$-th phase, $\boldsymbol{E}^{(i)}=\frac{1}{V_{i}} \int_{V_{i}} \boldsymbol{\varepsilon}^{(i)}(\mathbf{x}) d V$ (where $V_{i}$ the volume of the $i$-th phase or inhomogeneity), to the applied (average) macroscopic strain, $\overline{\boldsymbol{E}}$. By assuming a uniform temperature throughout the composite, the incremental localization relation (in which the strain concentration tensor is instantaneous and incorporates nonlinear effects [239]) of a phase has the form,

$$
d \boldsymbol{E}^{(i)}=\mathbf{A}^{(i)}: d \overline{\boldsymbol{E}}+\boldsymbol{\Theta}^{(i)} d T
$$

with $\boldsymbol{\Theta}^{(i)}$ being the thermal strain concentration tensor. The determination of the strain and thermal strain concentration tensors in the localization relations given by equation (4.1) is the key step in the construction of the homogenization law. Assuming a general incremental thermomechanical constitutive relation for the phases of the form

$$
d \boldsymbol{\Sigma}^{(i)}=\mathbf{L}^{(i)}: d \boldsymbol{E}^{(i)}+\boldsymbol{l}^{(i)} d T
$$

where $\boldsymbol{\Sigma}^{(i)}$ is the $i$-th phase average stress, $\mathbf{L}^{(i)}$ denotes the tangent stiffness tensor and $\boldsymbol{l}^{(i)}$ is the tangent thermal stress tensor of the $i$-th phase (typically taking the form $\boldsymbol{l}^{(i)}=-\mathbf{L}^{(i)}: \boldsymbol{\alpha}^{(i)}$ ), the effective composite tangent stiffness matrix is given as

$$
\overline{\mathbf{L}}=\sum_{i=0}^{N} c_{i} \mathbf{L}^{(i)}: \mathbf{A}^{(i)}
$$

with $c_{i}$ being the volume fraction of the $i$-th phase. The determination of thermal strain concentration tensors, $\boldsymbol{\Theta}^{(i)}$, and their effective counterpart, $\overline{\boldsymbol{\Theta}}$, may be found in terms of the mechanical concentration tensors and constituent thermomechanical properties as has been previously explored (especially in the context of thermal residual stresses in fibrous composites [121]) via schemes like the decomposition approach of Benveniste and Dvorak [48].

It is important to note, that the above relations are completely general. That is to say, no specific theory or micromechanical approximation is introduced in their formulation leaving them universal and applicable to any spatially uniform microstructure under uniform macroscopic loading. Additionally, although the strain concentration tensors, $\mathbf{A}^{(i)}$, are emphasized in the preceding relations, analogous forms in terms of the stress concentration tensors, $\mathbf{B}^{(i)}$, via a compliance (rather than stiffness) formulation may be found.

\subsubsection{Mean-Field Approaches}

Mean field estimates of the effective response of SMA composites have been given based on the MoriTanaka, self-consistent, and the second order variational methods. In the first two approaches, the composite is discretized into a finite (typically but a few) number of phases with specified responses, 


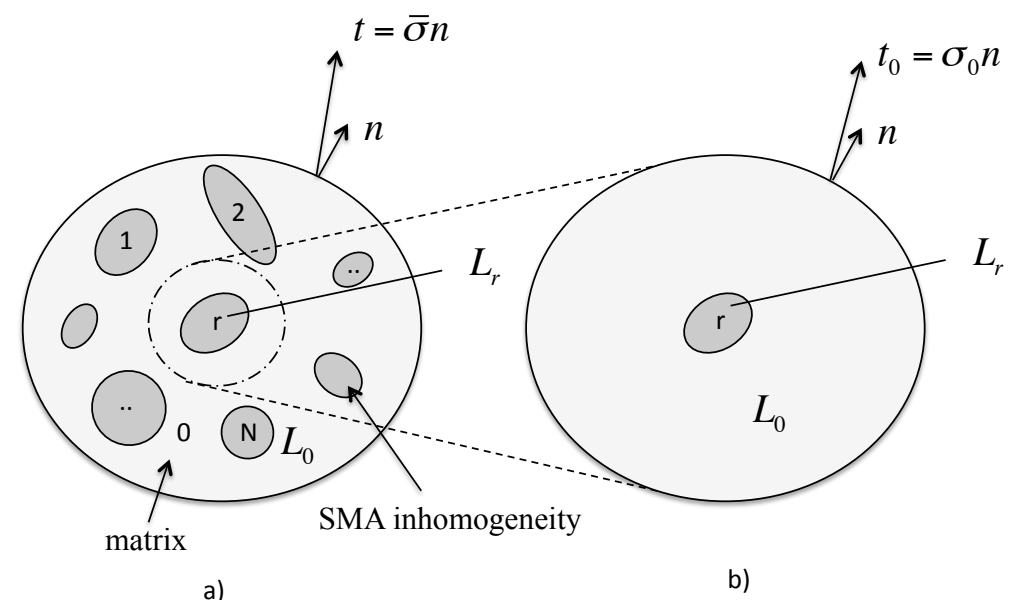

a)

Fig. 12: Illustration of the boundary value problem considered for analysis via Eshelby-based mean field approaches: (a) a composite comprised of $N$ distinct, ellipsoidal inhomogeniety phases embedded in a distinct matrix and (b) the Mori-Tanaka problem for the $r$-th phase. (CIOP Publishing. Reproduced from [252] by permission of IOP Publishing. All rights reserved.

properties, and orientation. A given phase is embedded into an infinite matrix and strain (or stress) concentration tensors are determined via the solution of boundary value problems $[128,305]$ as depicted in Fig. 12. With the Mori-Tanaka Method [303,47,305] the inhomogeneity is embedded in the matrix phase with the far-field conditions being that of matrix average. The self-consistent scheme [179], on the other hand, embeds the different phases in the effective, averaged material in an implicit scheme. Finally, the key idea of the second order variational method [76] is to write down a second-order Taylor expansion for the phase potentials about appropriately defined phase average strains. The resulting estimates, which are exact to second order in the contrast, involve interpolation of the nonlinear constitutive relations, and reduce the problem for the nonlinear composite to a linear problem for an anisotropic thermoelastic composite that can be solved via "traditional" mean-field approaches.

Below, several investigations utilizing the aforementioned mean-field estimates are briefly reviewed, organized by the applied scheme. In addition, in Table 10 these investigations are organized with respect to the composite material system.

Table 10: Mean-field approaches.

\begin{tabular}{lcrr}
\hline Methodology & SMA Reinforcement & Matrix & References \\
\hline Mori-Tanaka method & Fiber & Elastomer & {$[68,69,284]$} \\
$"$ & $n$ & Metal & {$[247,489]$} \\
$"$ & Particle & Ceramic & {$[460,57,58]$} \\
$"$ & $n$ & Porous SMAs & {$[366,124,125,312]$} \\
$"$ & - & Precipitated SMAs & {$[99,87,357,358]$} \\
Self-consistent method & - & Metal or elastomer & {$[407,94]$} \\
$"$ & Particle & Elastomer & {$[292]$} \\
Mori-Tanaka \& & Fiber & Precipitated SMAs & {$[98]$} \\
Self-consistent method & - & Concrete & {$[140]$} \\
High fidelity generalized method of cells & $n$ & Elastomer & {$[71]$} \\
Second order variational method & $"$ & & \\
\hline
\end{tabular}

Mori-Tanaka method - Boyd and Lagoudas [68,69] developed a micromechanical framework to predict the effective properties of SMA fiber-reinforced polymer matrix composites. The concept of effective transformation properties was introduced and the effective inelastic transformation strain 
and phase transition temperatures were derived using Mori-Tanaka concentration tensors. Moreover, it was demonstrated that SMA fiber/elastomer matrix composites can undergo the TWSME even though the SMA fiber can only undergo the OWSME. In their work, the Eshelby tensor was evaluated numerically, as in [150], since the inelastic transformation strain induces anisotropy in the tangent stiffness matrix. The ability to strengthen an aluminum matrix SMA composite via residual stresses and transformation toughening was demonstrated by Yamanda et al. [460] and Lee and Taya [247] via similar methodologies. The residual stress caused by the SME of predeformed fillers was shown to contribute significantly to the strengthening of this composite [460]. Moreover, it was shown that the magnitude and the direction of residual stresses can be controlled by pre-straining the composite. The model of Lee and Taya [247] predicted yield stresses of the SMA composite in good agreement with the experimental results by Hamada et al. [163]. The effective transformation characteristics of SMA composites considering different matrices and shapes was also studied by Lester et al [252] who highlighted the impact of the transformation-induced stress redistribution associated with a stiff matrix on the composite response.

In addition to "traditional" SMA composites, the Mori-Tanaka method has been used to develop constitutive models of porous and precipitated NiTi specimens. With respect to the former, Qidwai et al. [366] used an incremental formulation of the Mori-Tanaka method to account for random distribution of pores in the matrix. The obtained results were shown to compare well with unit cell finite element method results in predicting the isothermal elastic material properties and pseudoelastic response under axial and out-of-plane shear loading. However, the transformation results differ under transverse and in-plane shear loading. Entchev and Lagoudas [124,125] extended this approach to incorporate transformation-induced plasticity (TRIP) strains in the matrix phase (Fig. 13a). Expressions for the effective elastic and tangent stiffness tensors were presented as well as an expression for the evolution of the effective inelastic strain. Furthermore, the authors addressed one other key issue of porous SMAs produced from elemental powders - determination of the constitutive properties of the matrix phase. A detailed procedure to this end was presented and discussed. For the latter case, the response of bulk NiTiNb was modeled and simulated as a composite comprised of $\mathrm{Nb}$ inhomogeneities, of a relatively small volume fraction $(\sim 9 \%)$, in a homogeneous NiTi matrix [87,357,358]. The obtained results highlighted the effect of niobium precipitates on the thermomechanical behavior of $\mathrm{NiTiNb}$, and particularly on the corresponding hysteresis size which was shown to increase due to the plastic deformation of the $\mathrm{Nb}$ precipitates.

To consider the interaction between the austenitic and martensitic SMA phases and the matrix, three-phase methods were also developed to study the response SMA composites. Lu and Weng [284] used such a two-level micromechanical theory to study the influence of shape and volume concentration of SMA inclusions on the overall response of a SMA-reinforced composite. The first level exists on the smaller SMA level, in which, under the action of stress, parent austenite may transform into martensite. The second level is on the larger scale consisting of the metastable SMA inclusions and a polymer matrix. The Mori-Tanaka method was utilized to derive both the change in the mechanical potential energy due to phase transformation in the micro level and for determining the average stress in the SMA inclusions and the overall strain of the SMA-reinforced composite on the macroscopic level for given inclusion shapes. The authors showed that fibrous SMAs in NiTi-reinforced elastomer-matrix composites represent the most advantageous choice when the design criteria are superior stiffness, easier transformation, and higher damping capacity (Fig. 13b). Birman [57,58] developed a similar numerical scheme to investigate the effect of randomly distributed SMA spheroidal particles on the effective thermomechanical properties [57] and stress-strain loop reflecting hysteresis of fiber-reinforced polymer composites and on the stress states of SMA particle reinforced MMCs [58]. The Mori-Tanaka homogenization technique was used at both levels to develop relations between the properties of the composite material and the applied stresses. The author showed that the energy dissipation within a MMC with spherical SMA inclusions is greater to that observed in composites with SMA fibers under transverse load. A three-phase micromechanical model based on the Mori-Tanaka scheme was also derived by Zhu and Dui [489] to investigate the effect of fiber shape on the overall response of SMA/elastic-plastic matrix composite and on the residual stresses. The obtained results were in good agreement with experimental results presented by Hamada et al. [163].

Nemat-Nasser et al. [312] presented a micromechanical model to predict the stress-induced phase transformation of a porous shape-memory alloy with small porosity ( $12 \%$ ). This model was based on the modified equivalent inclusion method for a three-phase system, with the parent phase (austenite) 


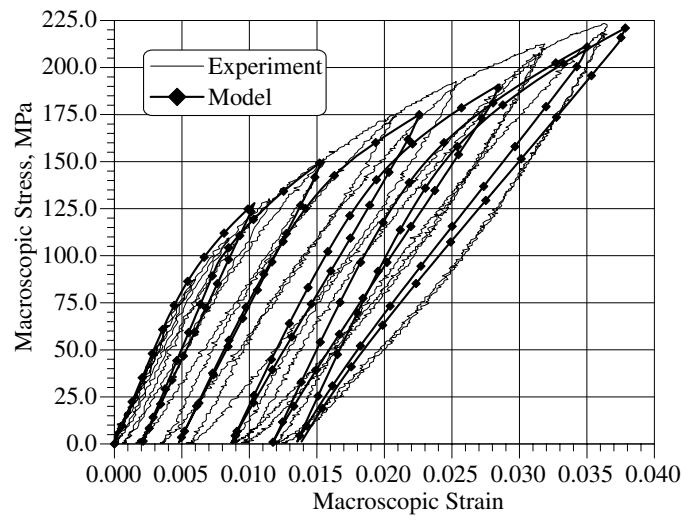

(a) Stress-strain response of a large pore porous NiTi SMA bar incorporating TRIP - comparison between the experimental results and the model simulation. Reprinted from [125] with permission from Elsevier.

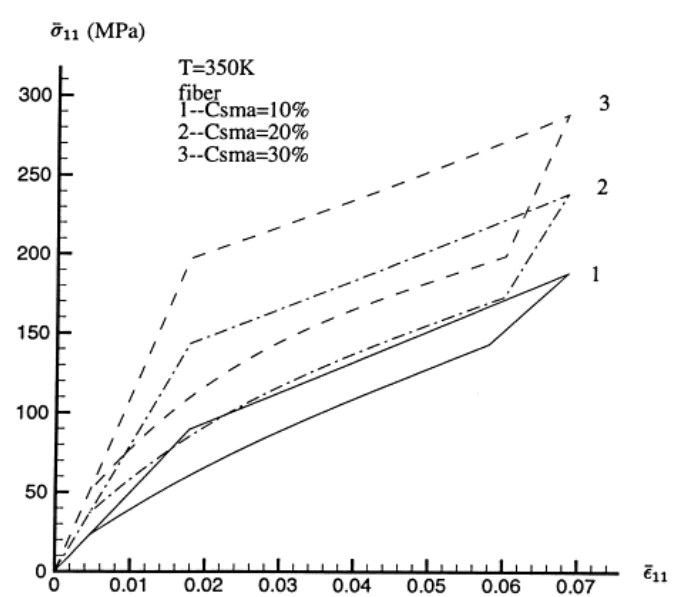

(b) Stress-strain behavior of an elastomermatrix composite reinforced with SMA fibers at three selected volume concentrations. Reprinted from [284] with permission from Elsevier.

Fig. 13: Stress-strain response of porous SMAs and SMA/elastomer composites.

as the matrix and the product phase (martensite) and the voids as the embedded inclusions, reversing the roles of austenite and martensite during the reverse transformation from fully martensite to fully austenite phase, and equilibrium thermodynamics. This three-phase micromechanics model is a special case of the multi-phase composite model (MPCM), and can be obtained as a special case of MPCM by assuming that the martensite phase and the pores are ellipsoids with common aspect ratios and orientations. The overall stress-strain behavior of the porous SMA and its temperature dependence were obtained using the martensite volume fraction and found to be in general agreement with quasistatic experimental data from porous $(\sim 12 \%)$ NiTi synthesized by spark-plasma sintering.

Self-consistent method - A micromechanical model based on Hill's incremental self-consistent scheme was developed by Cherkaoui et al. [94] to determine the mechanical response of two SMA composites: aluminum and soft (polymer) matrices with spherical SMA particulates (Fig. 14). In this micromechanics modeling, the internal stress and strain in both matrix and SMA and their evolution were derived as functions of the externally applied thermomechanical loading and the degree of phase transformation in SMA. The model was used in a subsequent paper by Song et al. [407] to investigate the influence of the shape and volume fraction of SMA on the overall behavior of the composite as well as on the internal stress and strain evolution. A strong dependence of thermomechanical response of composite on spatial arrangement of SMA inclusions, the constitutive parameters of matrix and SMA, the stress state, loading history and temperature, etc, was shown. Marfia and Sacco [292] used a self-consistent Eshelby formulation for simulating the response of SMA/elastic matrix composites and compared the obtained results to finite element results of periodic microstructures to validate them. A three-dimensional model for the SMA material was adopted, that takes into account the thermomechanical behavior and the asymmetric tension-compression response.

Mori-Tanaka combined with the self-consistent method - Collard and Ben Zineb [98] recently adopted a two-scale homogenization methodology to simulate the thermomechanical behavior of polycrystalline SMA containing elastic precipitates. The effective behavior of grains, i.e., SMA single crystals containing elastic precipitates, was obtained using an incremental Mori-Tanaka approach [99], while the effective response of the polycrystal was obtained using an incremental self-consistent scheme, similar to [344]. The obtained results showed an increase of the hardening-like slope of the effective behavior and the obtained transformation strain for a given stress when the volume fraction of hard precipitates increases in accordance with experimental evidence.

Second order variational method - This method, developed for nonlinear composites by Ponte Castaneda and Suquet [76] was applied to SMA/elastic matrix composite actuators by Briggs and 


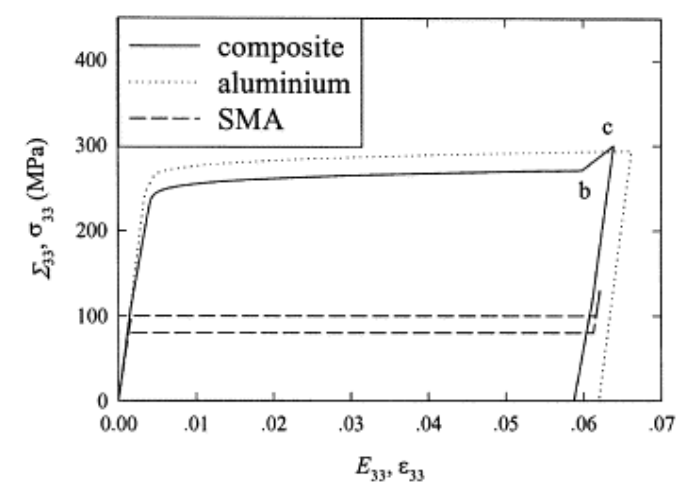

(a) The axial stress strain curves of composite, aluminum and SMA under uniaxial tension.

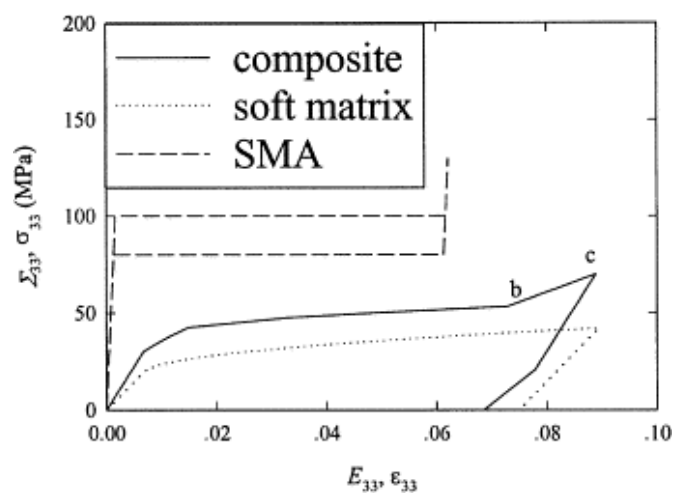

(b) The axial stress strain curves of composite, soft matrix and SMA under uniaxial tension.

Fig. 14: Predictions of a micromechanical model based on Hill's incremental self-consistent scheme. Reprinted from [94] with permission from Elsevier.

a

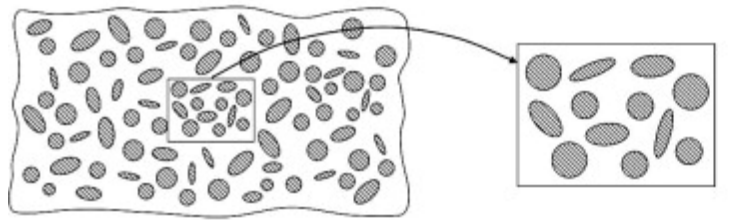

b

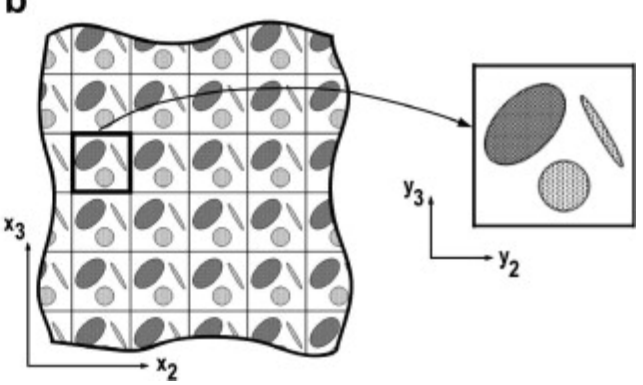

Fig. 15: Illustration of (a) the RVE, and (b) UC concepts. In the former, a sufficient volume is identified such that the statistical ensemble is macroscopically homogeneous while in the latter a repeating microstructure unit is identified. Reprinted from [354] with permission from Elsevier.

Ponte Castaneda [71] with the objective to explore possible performance optimization by varying the possible "configurations" of the composites. Performance was characterized by maximum contraction and largest bandwidth of the system response as a whole. One of the conclusions was that it may be possible to have large percentage contraction and better response time by choosing a soft matrix but including a pretension on the fibers, with this improvement having an associated cost in energy requirements for heating.

\subsubsection{Full-Field Approaches}

Micromechanical analyses of spatially uniform heterogeneous media which explicitly account for the actual microstructural details at the local level may be grouped into representations that involve the concepts of periodicity and statistical homogeneity based on the concomitant concepts of UC and RVE, respectively (for the definitions of UC and RVE, we refer to [116]). Examples differentiating UC and RVE domains are presented in Fig. 15.

With regard to periodic media, a brief outline of the mathematical homogenization theory is first given which involves solution of the unit cell boundary-value problem. In order to deal with two different length scales associated with the macroscopic and microscopic behaviors, the global coordinate, $\boldsymbol{x}$, and the local one, $\boldsymbol{y}$, are defined respectively. These coordinates are related to each other by a small positive real parameter, $\epsilon$, such that $\boldsymbol{y}=\boldsymbol{x} / \epsilon$. 
The most important and essential postulate in the homogenization method is a multiscale representation of the displacement $\boldsymbol{u}$

$$
\boldsymbol{u}^{(\epsilon)}(\boldsymbol{x}, \boldsymbol{y})=\boldsymbol{u}^{(0)}(\boldsymbol{x}, \boldsymbol{y})+\epsilon \boldsymbol{u}^{(1)}(\boldsymbol{x}, \boldsymbol{y})+O\left(\epsilon^{2}\right)
$$

Inserting the above equation to Navier's equations and identifying powers of $\epsilon$ leads to a cascade of equations for each term $\boldsymbol{u}^{(i)}$. in the context of linear elasticity (mathematical homogenization of inelastic heterogeneous media is rather complicated [139]) and in the absence of body forces and dynamic effects, inserting (4.4) into the Navier's equations

$$
\nabla_{\boldsymbol{x}} \cdot\left[\mathbf{C}(\boldsymbol{y}): \nabla_{\boldsymbol{x}} \boldsymbol{u}(\boldsymbol{x}, \boldsymbol{y})\right]=0
$$

and by arranging them against the various orders of $\epsilon$ using the chain rule for the spatial gradient, $\nabla_{\boldsymbol{x}} \rightarrow \nabla_{\boldsymbol{x}}+\frac{1}{\epsilon} \nabla_{\boldsymbol{y}}$, the following equations are obtained. The $\epsilon^{-2}$ equation $\boldsymbol{u}^{(0)}(\boldsymbol{x}, \boldsymbol{y})=\boldsymbol{u}^{(0)}(\boldsymbol{x})$, the $\epsilon^{-1}$ equation which represents the local problem and sets up the boundary-value problem for the unit cell in terms of the 1st-order displacement field components $\boldsymbol{u}^{(1)}(\boldsymbol{x}, \boldsymbol{y})$, and the $\epsilon^{0}$ equation

$$
\nabla_{\boldsymbol{x}} \cdot\left[\overline{\mathbf{C}}: \nabla_{\boldsymbol{x}} \boldsymbol{u}^{(0)}(\boldsymbol{x})\right]=0
$$

which represents the global problem. The last equation is an effective Navier's equation given in terms of the homogenized modulus

$$
\overline{\mathbf{C}}=\frac{1}{V_{U C}} \int_{V_{U C}}\left[\mathbf{C}(\boldsymbol{y})+\mathbf{C}(\boldsymbol{y}): \nabla_{\boldsymbol{y}} \mathbf{N}(\boldsymbol{y})\right] d V
$$

where $\mathbf{N}(\boldsymbol{y})$ plays the same role as the local strain concentration tensor and is obtained from the solution of the $\epsilon^{-1}$ order equation, i.e., the unit-cell boundary-value problem, as follows

$$
\boldsymbol{u}^{(1)}(\boldsymbol{x}, \boldsymbol{y})=\mathbf{N}(\boldsymbol{y}): \nabla_{\boldsymbol{x}} \boldsymbol{u}^{(0)}(\boldsymbol{x})
$$

The solution of the unit cell boundary-value problem is typically generated using the finite-element method in terms of the 1st-order displacement field components $\boldsymbol{u}^{(1)}(\boldsymbol{x}, \boldsymbol{y})$, although in principle any solution approach can be employed. Analyses of unit cell boundary-value problems are conducted both outside and within the asymptotic homogenization theory's framework. There are other representations that follow the homogenization theory's framework described above wherein the global coordinates $\boldsymbol{x}$ describe the average response of the entire periodic array, while the local coordinates $\boldsymbol{y}$ are associated with the unit cell but do not explicitly introduce the small parameter $\epsilon$ in the displacement field decomposition. Such approaches will still be referred to as homogenization models below. Such methodologies include the high fidelity generalized method of cells and computational homogenization approaches.

Regarding statistical homogeneous materials, there are various RVE definitions [178, 189, 209,117]. The classical RVE is defined as the smallest material volume of which the response does not depend on the applied boundary conditions $[178,189]$. In another definition, based on the knowledge of the statistical nature of the microstructure, the RVE can be regarded as a volume $V$ sufficiently large to be statistically representative of the material, i.e., it includes a sampling of all microstructural heterogeneities that occur in the material [209]. Dragan and Willis [117] defined an RVE as "the smallest material volume element of the composite for which the usual spatially constant (overall modulus) macroscopic constitutive representation is a sufficiently accurate model to represent mean constitutive response". Therefore, it can be concluded that there is a priori no systematic quantification of the RVE sizes, and that results will depend on many combinations of factors (material structure, volume fraction of heterogeneities, etc.). The intrinsic difficulty of simultaneously satisfying homogeneous displacement and traction boundary conditions, necessary in fulfilling the RVE requirement for subvolumes with random microstructures, has led to the concept of periodization of random media. Numerical estimates of the effective properties of random heterogeneous materials are more accurate when periodic boundary conditions are applied than when homogeneous strain or stress boundary conditions are considered [209]. Kanit et al [209] showed that the effective physical properties of a statistical homogeneous material can be determined either by a few number of measurements on large RVE volumes (large number of particles for a given volume fraction), or by many realizations for small volumes of material. However, the mean values computed on small RVE volumes cannot represent the effective response 
for the composite material even when using periodic boundary conditions and a sufficient number of realizations. This size of the RVE must be established for each material system, phase content, and loading mode.

Below, a review of SMA composites investigations using the notions of periodic and statistical homogeneous materials is given. These investigations are gathered in Table 11, organized with respect to micromechanics scheme and composite material system.

Table 11: Full-field approaches.

\begin{tabular}{lccr}
\hline Methodology & SMA Reinforcement & Matrix & References \\
\hline Unit cell & - & Porous SMAs & {$[366]$} \\
$"$ & Fiber & Elastomer & {$[3]$} \\
$"$ & $"$ & Elastomer or Metal & {$[109,110]$} \\
& $"$ & Nylon fiber / elastomer & {$[399]$} \\
Homogenization & $\#$ & (Woven composite) & {$[177,291]$} \\
$"$ & $\#$ & Elastomer & {$[18,142,141]$} \\
$"$ & $"$ & Polymer & Metal \\
$"$ & $"$ & Concrete & {$[18,290,142,141,82]$} \\
$"$ & - & SMA honeycombs & {$[144]$} \\
RVE analysis & - & Precipitated SMAs & {$[39]$} \\
$"$ & - & Porous SMAs & {$[365,335,17]$} \\
$"$ & Particulate & MAX Phase & {$[93,253]$} \\
& Fiber & Metal & {$[228]$} \\
\hline
\end{tabular}

Homogenization and UCs - Qidwai et al. [366] adopted the UC concept to account for periodic distribution of pores in the SMA matrix in an effort to predict the constitutive response of porous SMAs. Cylindrical and spherical shapes were considered as approximations of open and closed pores, respectively (Fig. 16). Similar approaches were also used with NiTi fiber reinforced elastomer [3] and elastic-plastic metallic $[109,110]$ matrix composites. In the latter work, the effects of fiber volume fraction, off-axis angle, matrix material and pre-strain on the micro-thermomechanical behavior of composites with embedded SMA fibers subjected to various multi-axial proportional/non-proportional loadings were examined. In the former, the numerical scheme was used to evaluate the stress field in the composite as consequence of the shape-memory effect of embedded SMA wires. In the paper of Sepiani et al. [399], a theoretical study of the in-plane behavior of SMA Woven Composites (SMAWC) under biaxial loading was presented by developing an integrated micromechanical constitutive model. The framework of the approach consisted of the geometric model of a unit cell, and the strain energy based constitutive equations under large deformation. The deflection of the structure, subjected to uniform biaxial loading was studied numerically showing the significance of the SME on the behavior of plain woven flexible fabric composites.

Herzog and Jacquet [177] adopted a homogenization method based on multiple scale asymptotic expansion to predict the response of SMA wire reinforced composites of various configurations. The effects of the inelastic deformation of fibers on the overall behavior of unidirectional NiTi composite were numerically examined for loading and unloading in the longitudinal direction using the developed three-dimensional model. The high-fidelity generalized method of cells [1] was used extensively by Freed, Aboudi, and coworkers to study a number of different SMA composite systems while incorporating full thermomechanical coupling [143,144,140,142,141]. Of particular interest was the ability of prestressed SMA fibers to strengthen concrete materials considering both a damage-based [143] and coupled damage-plastic [140] formulation for the concrete phase. Through these investigations, it was shown that fiber shape was insignificant if non-zero thermal expansion coefficients were used and higher activation temperatures lead to larger beneficial prestresses [143]. Yield surfaces of the resultant material were also constructed and in the damage-plasticity case it was demonstrated that increases in the activation temperature produced decreased residual plastic strain [140]. The effect of the thermomechanical coupling over repeated cycling was considered for both aluminum and epoxy matrix composites and a more pronounced effect was observed in the Al matrix due to the associated heat transfer [142]. Axial, transverse, and axial-transverse loadings were investigated for the same matrices 


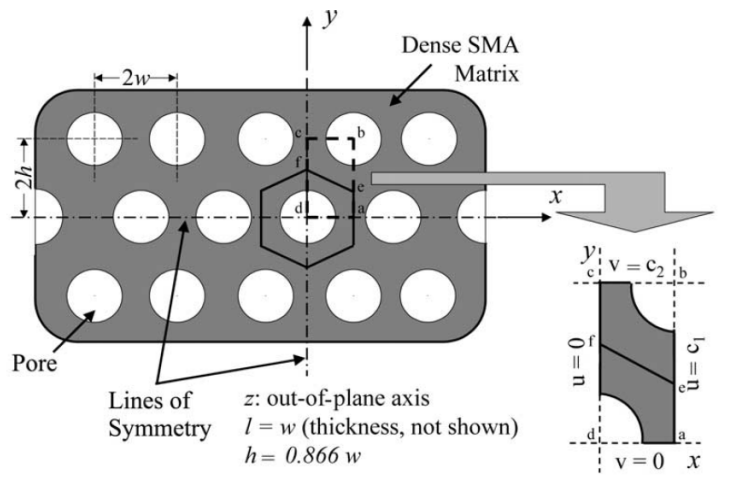

(a) Cross-sectional schematic of periodic hexagonal arrangement of open pores (cylinders) in a dense SMA matrix. The blown-out image is the cross-section view of the unit cell used in the analysis.

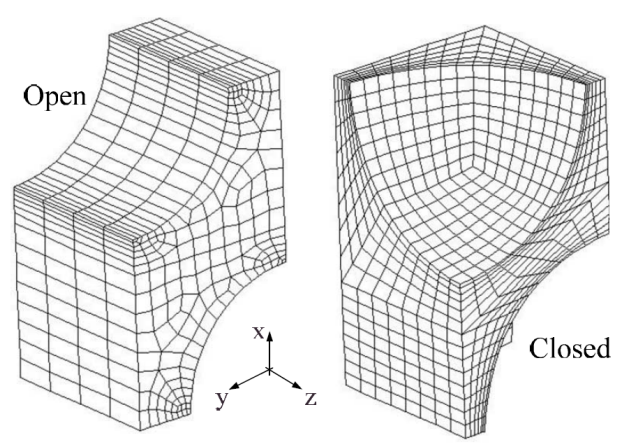

(b) Finite element meshes employed in the analyses of periodic open and closed pore SMA, respectively, containing pore volume fraction of 0.4 .

Fig. 16: Finite element analysis of porous SMAs. Reprinted from [366] with permission from Elsevier.

on both the OWSME and TWSME effects [141]. In a different fashion, the response of honeycomb foams with hexagonal, circle, re-entrant, and triangle topologies were also explored. Generally, a similar macroscopic response (both in terms of pseudoelasticity and SME) were observed but the triangle configuration was found to have a stiffer, more preferable response. Aurrichio and Petrini [18] also explored the effect of the thermomechanical coupling on a number of hybrid composites (both metal and various polymers).

A multi-scale approach was adopted by Marfia and co-workers [290,291] in which the constitutive relationship was determined solving at each nonlinear iteration of each time step for each integration Gauss point a nonlinear homogenization problem, based on the self-consistent technique. The developed nonlinear numerical procedure is computationally efficient and it show good convergence properties. The buckling response of SMA composite plates and the response of a cantilever plates through various loadings were investigated. Chatzigeorgiou et al. [82] proposed a homogenization framework for periodic composites with SMA constituents under quasi-static thermomechanical conditions. The methodology was based on the step-by-step periodic homogenization, in which the macroscopic and the microscopic problems of the composite are solved simultaneously. Their results for multilayered materials with SMA and metallic material (elastic-plastic) components showed complex macroscopic response, with significant interactions between transformation and plasticity.

RVEs - Baxevanis et al. [39] developed a microscale-informed model that takes into account the precipitate-shape memory performance relations and allows for the evaluation of the effective thermomechanical properties that can be used to simulate the structural response of such materials. The model considers the structural effect of the precipitates (coherency stresses due to the lattice mismatch between the precipitates and the matrix material and precipitate volume fraction), as well as the effect of the Ni-concentration gradient in the matrix, resulting from Ni-depletion during precipitate growth (Fig. 17). The predictive capability of the model is tested for Ni-rich NiTi material systems of different composition and heat treatment and good agreement was shown [104].

In addition to precipitated systems, RVE analysis has also been used to study porous NiTi specimens. Specifically, 3D RVEs have been constructed using a statistically-informed (determined via X-ray microtomography) [365] and random [335,17] approach. The former investigation by Qidwai and DeGiorgi [365] focused on the dynamic behavior of porous NiTi while the latter two investigations considered retained martensite in the constitutive formulation to explore the development of permanent deformations [335] and study the pressure dependence of the porous response to develop and calibrate a porous SMA constitutive model [17], respectively. Recent investigations have also utilized RVE methodologies to explore the response of SMA-MAX phase composites. Specifically, Cheng et al. [93] constructed RVEs (Fig. 18) using SEM images of a $\mathrm{NiTi}-\mathrm{Ti}_{3} \mathrm{SiC}_{2}$ composite to determine the effective thermal properties over a large temperature range. Importantly, they noted the impact of the interphases on the thermal properties. Image-based approaches leveraging X-ray microtomography 


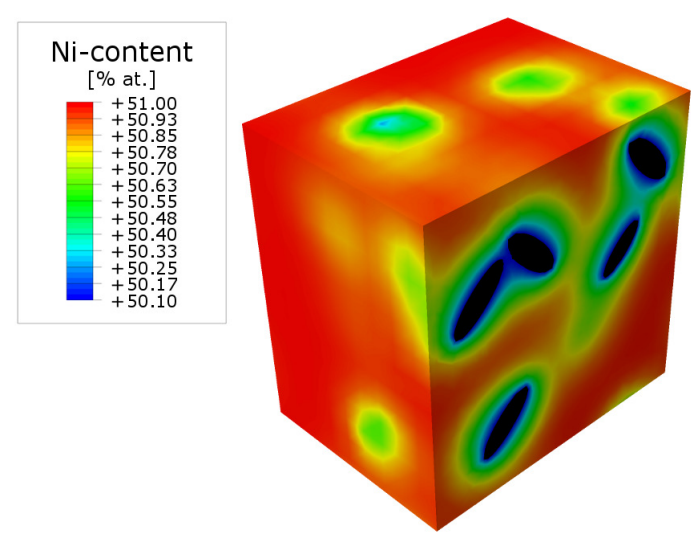

(a) Ni-concentration (at.\%) field in the matrix. The volume fraction of the precipitates is $3 \%$.

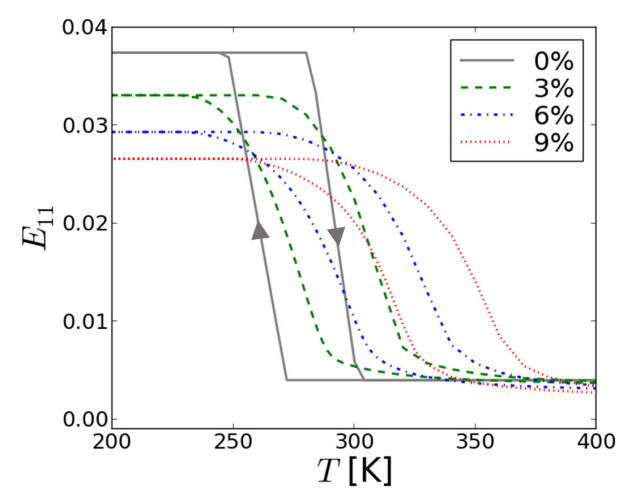

(b) Comparison of the effective strain in the direction of loading vs temperature responses of $0 \%$, $3 \%, 6 \%$, and $9 \%$ volume fraction precipitated materials for a thermal cycle under a constant uniaxial loading at $200 \mathrm{MPa}$.

Fig. 17: Finite element analysis of precipitated Ni-rich NiTi material systems. Reprinted from [39] with permission from Springer.

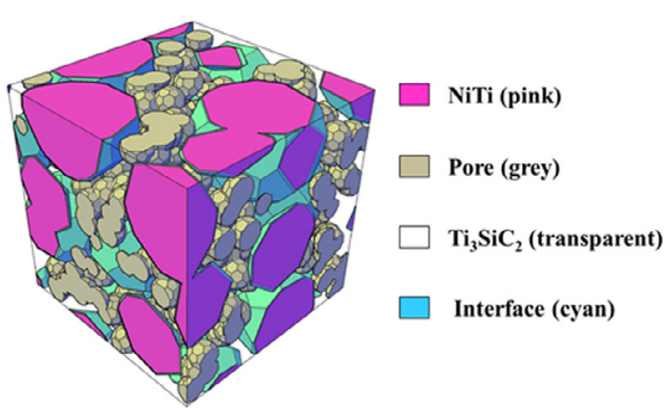

(a)

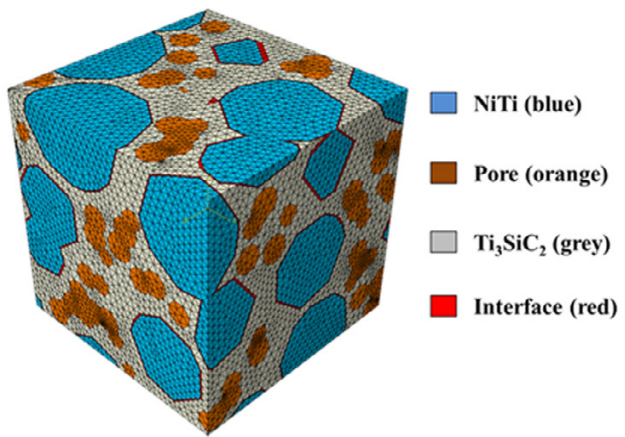

(b)

Fig. 18: Detailed 3D, multiphase (a) numerical microstructure and (b) finite element mesh used for the thermal analysis of a $\mathrm{NiTi}-\mathrm{Ti}_{3} \mathrm{SiC}_{2}$ composite. Reprinted from [93] with permission from Elsevier.

have also been used to create 3D RVEs of a $\mathrm{NiTi}-\mathrm{Ti}_{2} \mathrm{AlC}$ composite and explore the effective thermomechanical response, notably the development of the residual stress state [253]. Finally, a recent effort by Kohlhaas and Klinkel [228] looked at the development of an $\mathrm{FE}^{2}$ approach for studying RVEs with various, detailed microstructures. They focused on SMA wire reinforced structures and reported similar results between a discrete and $\mathrm{FE}^{2}$ model thereby demonstrating the capability of the latter to analyze SMA composite problems.

\subsection{Structural analysis tools}

Apart from micromechanics-based methodologies that allow for the determination of the overall nonlinear behavior of SMA composites, several other analytical and numerical tools have been developed for the structural analysis of SMA composites that can be broadly categorized in the following groups:

- Numerical tools

- SMA elements and the way they interact with the host structure are modeled directly and separately from the structure itself 
- SMA elements are modeled as a force acting on the rest of the structure, whose value is obtained from constitutive models or experimental measurements

- Analytical tools

- Analytical tools based on shear-lag models, stress functions, first-order shear deformation theory, etc.

Below, the various investigations to be discussed (Table 12) are presented in terms of the structural response they investigate in lieu of methodology used as the former is found to be more advantageous. Therefore, the investigations reviewed are gathered into four major groups: the first group consists of investigations that study the actuation response of SMA composites, the second one, of those that study damage suppression, the third one, named "Damping", includes investigations related to buckling and vibration control, and the fourth one consists of the design related investigations.

Table 12: Structural analysis.

\begin{tabular}{|c|c|c|c|}
\hline Structural response & SMA Reinforcement & Matrix & References \\
\hline Actuation performance and response & Fiber & Glass fiber/epoxy & {$[207,387,149,427,97]$} \\
\hline$"$ & " & Carbon nanofiber/polymer & {$[107]$} \\
\hline$"$ & $"$ & Graphite/epoxy & {$[465]$} \\
\hline$"$ & $"$ & Elastomer & {$[25,90]$} \\
\hline$"$ & Ribbon & $"$ & {$[62]$} \\
\hline Damage suppression & Fiber & Metal & {$[73]$} \\
\hline$"$ & $"$ & Elastomer & {$[249,6,443,306,444,445]$} \\
\hline$"$ & $"$ & Glass fiber/epoxy & [65] \\
\hline$"$ & $"$ & Carbon fiber/epoxy & {$[295,220]$} \\
\hline Damping & $"$ & $"$ & {$[2]$} \\
\hline$"$ & $"$ & Graphite/epoxy & {$[283,215,12,13,11,390,190]$} \\
\hline$"$ & Layers & Elastomer & {$[479,480,109]$} \\
\hline Design & Fiber & Elastomer & {$[349-351]$} \\
\hline$"$ & Fiber & Glass fiber/epoxy & {$[340,339]$} \\
\hline
\end{tabular}

Actuation performance and response - Jung et al. [207] investigated the deformation behavior of a SMA wire-embedded composite as a function of the composite width per SMA wire, composite thickness, and the eccentricity of the SMA wire. A curved morphing composite structure was fabricated to confirm the results of the numerical simulation. Ryu et al. [387] used finite element analysis (FEA) to predict the deformation behavior of SMA wire-embedded hybrid composites and compared the numerical results against experimental ones. The FE models was then used to achieve a favorable tradeoff between the stiffness and the achievable deformation of the structure. Responses of hybrid beams in clamped-clamped conditions were further studied through finite element techniques. Gao et al. [149] presented simulations of adaptive stiffening and shape control of SMA hybrid composites compared the numerical results to experimental results. The deflection versus temperature response of a cantilever beam demonstrated the efficient control authority of the embedded SMA. Turner and Patel [427] examined the response of a laminated composite composed of unidirectional pre-impregnated (prepreg) layers with SMA actuators embedded within the composite pre-preg laminae. The thermoelastic, vibration, and deflection control examples presented in this study demonstrate the utility of SMAHC structures for vibroacoustic, aerodynamic, and aeroacoustic applications. Special SMA elements were also used in conjunction with a nonlinear plate implementation to investigate both the change in stiffness and actuation characteristics of SMA wires inserted in a plate structure and compared to experiments [107]. The proposed model is simple and flexible, so it can be a useful tool for the design process of such applications and the choice of optimal control schemes. The response of hybrid cantilever composite plate was analyzed by Cho and Ree [97] using a nonlinear shell finite element implementation. The obtained results show the ability of the suggested procedure to compute the thermomechanical behavior of such composites and demonstrated good agreement with experimental results from previous studies (Fig. 19). Bodaghi et al. [62] presented a study of the thermomechanical behavior of rectangular shape adaptive composite plates with surface-bonded SMA ribbons. The structural model was based on the first-order shear deformation theory and von Kármán kinematic nonlinearity. The implications 


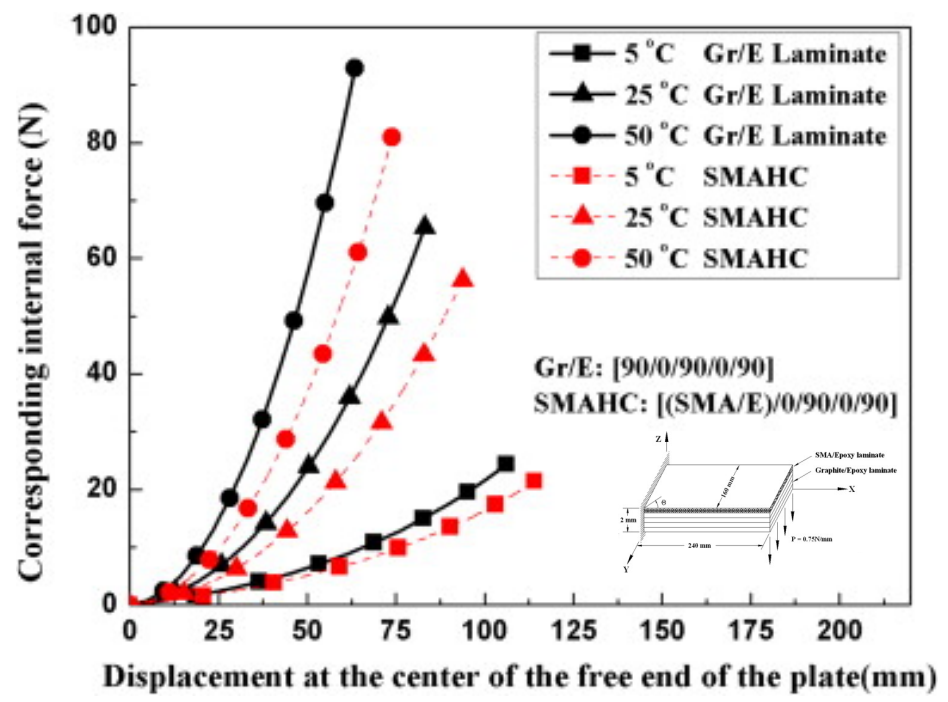

Fig. 19: Actuation response of a hybrid SMA composite presented in terms of deflection at the center of the end edge vs. corresponding internal force. The geometry and boundary conditions of the SMAHC plate are shown as an insert. Reprinted from [97] with permission from Elsevier.

of the pre-strain state, temperature, length and arrangement of the SMA ribbon actuators, as well as of boundary conditions of the plate on the thermomechanical behavior of shape adaptive composite plates were examined through a parametric study. Moreover, Balapgol et al. [25] developed a finite element model for the displacement and time response of a thermally driven SMA laminated actuator based on the first order shear deformation theory. The numerical results demonstrated that the input power, heat sink strength, thermal conductivity and thickness of the elastomer layer play important roles in controlling the time response of the SMA laminated actuator.

Yongsheng et al. [465] presented closed-form solutions for SMA fiber hybrid composite box beams with extension-twist or bending-twist coupling that is in the form of a cantilever beam. The variational asymptotic method was used to formulate the force-deformation relationships equations accounting for the presence of active SMA fibers distributed along the cross-section of the beam. The effects of the volume fraction of the SMA fiber, the martensitic residual strain, and ply angle were addressed. Numerical results showed that significant static deformations occur during the phase transformation due to SMA actuation. Chen et al. [90] analyzed the stress distribution in a thick fiber-reinforced composite tube structure incorporating an SMA by considering the material characteristics of the SMA and adopting the 3-D generalized plane strain-elasticity theory. The through-thickness stress distribution tendency and the reduction in the maximum stress were investigated by adopting different SMA layer arrangements and different SMA activation temperatures.

Damage Suppression - Burton et al. [73] built a finite element model of an SMA wire reinforced elastic-plastic matrix composite and demonstrated the self-healing capability of SMA composites via these simulations. Results show that the wires are able to pull the crack faces back together thereby healing the matrix under certain conditions. Use of pre-strained SMA wires in the composite is critical for a closure force to develop and adequate matrix material softening is required during heating in order for the crack faces to heal across the full width of the crack. Lei et al. [249] used a cohesive zone model to simulate the interface between SMA fibers and matrix in randomly generated short-fiber microstructures. The effects of interfacial debonding process, fiber ratio and ambient temperature on the response of the composites were discussed. Antico et al. [6] presented a finite element model in which the interface was modeled using a cohesive zone model that accounts for energy dissipation at the debonding front and inelastic deformation in a NiTi wire during pull-out. They demonstrated that residual stresses from the molding process significantly influence mode mixity at the debonding front. Bor et al. [65] studied the generation of through thickness internal stresses in a continuous fiber-reinforced composite material with embedded SMA wires. During a healing treatment SMA wires 
distributed in the out-of-plane direction compress the composite material enforcing a matrix delamination to close. In this way the development of an intimate contact zone at the delamination interface is stimulated, assisting thermal healing processes. Meo et al. [295] investigated the low-velocity impact damage behavior of carbon fiber/epoxy composite plates embedded with pseudoelastic SMA wires. The results show that for low velocity impact, embedding SMA wires into composites lead to an increase of the damage resistance of hybrid composites structures when compared to conventional composites structures. Kim et al. [220] studied the effects of SMA thin films embedded in composite plates for improving damage resistance of composite structures. Several effects, such as pre-strain, temperature, and position of SMAs were numerically investigated.

Wang and $\mathrm{Hu}$ [443] proposed an analytical model to examine the stress transfer for a SMA fiber pulled out from an elastic matrix in which the stress distributions were determined by a shear-lag method. The numerical calculations show that compared to elastic fiber, the transformation in SMA fiber lowers largely the axial stress, however, the radial stress is increased significantly due to the lateral contraction induced by transformation. Wang et al. [444,445] developed closed-form solutions for the internal stress in SMA fiber/matrix composites using the stress function method combined with the principle of minimum complementary energy approach in order to analyze the internal stress distributions during fiber pull-out and the thermal loading process. The entire range of axisymmetric states of stresses in the SMA fiber and matrix were developed. The results indicate substantial variation in stress distribution profiles for different activation and loading scenarios. In the work of Murasawa et al. [306], a constitutive relationship was proposed for SMA composites based on the shear-lag model. The main conclusions of this work were the following: composite shrinkage and compressive residual stress in the matrix increase with increasing aspect ratio and fiber volume fraction after heating.

Damping - The FEA of Ibrahim et al. [190] studying supersonic panel flutter and thermal buckling characteristics of SMA hybrid composite plates was based on the first-order shear deformable plate theory. Results showed that SMA fiber embeddings can be very useful in thermal buckling and flutter control through suppressing thermal postbuckling deflections, and enlarging the flat and dynamically stable performance domains at elevated temperatures. Samandpour et al. [390] (and Asadi et al. [11]) used similar methodologies to obtain buckling temperature and fundamental natural frequencies (nonlinear thermal instability of moving sandwich plates subjected to a constant moving speed) of a sandwich plate with embedded pre-strained SMA fibers in temperature dependent laminated composite (functionally graded) face sheets. The numerical results presented in both papers provided an insight into the effects of pre-strain, volume fraction and location of the SMA fibers, lamination scheme and geometrical parameters on the dynamic behavior of sandwich plates. Alebrahim et al. [2] investigated the vibration response of SMA hybrid composites. They concluded that SMA wires could be greatly effective in repairing flaws, improving natural frequency, and reducing deflection in composite beam-like structures. Finally, Damanpack et al. [109] investigated the vibration control capability of SMA composite beams subjected to impulsive loads. They concluded that SMA layers with high pre-strain at low temperatures have a passive vibration control capability and yield a better efficiency in comparison with pseudoelastic SMA layers.

Lu et al. [283] developed a theoretical model for the bending of a laminated beam bonded with an SMA fiber reinforced layer and provided the relationships between bending moment, curvature and temperature. The work of Zhang and Zao [479,480] used analytical and numerical examples to study buckling, post-buckling, free vibration and forced vibration SMA composite beams. Furthermore, the possible error sources of the composite natural frequencies due to modeling SMA wires as axial forces or an elastic foundation and anisotropy were discussed. The response of an SMA hybrid composite subjected to an impact loading was investigated by Khalili et al. [215] using the classical first-order shear deformation theory as well as the Fourier series method to solve the governing equations of the composite plate analytically. A linearized Hertzian contact model was used in the impact analysis of the laminated hybrid composite plate. The results indicated that some of the geometrical and the material parameters like the volume fraction, the orientation and the through thickness location of the SMA wires, the longitudinal to transverse elastic modulus ratio of the plate and the material type of composite medium are important factors affecting the impact process and the design of the structures. Asadi and coworkers $[12,13]$ studied nonlinear free and forced vibrations of a fully clamped hybrid composite beam reinforced with SMA fibers and different lay-ups were analytically analyzed. Based on the Euler-Bernoulli beam theory together with kinematic nonlinearity in the von-Kármán sense and employing the Hamilton principle, the equations of motion were established for hybrid- 


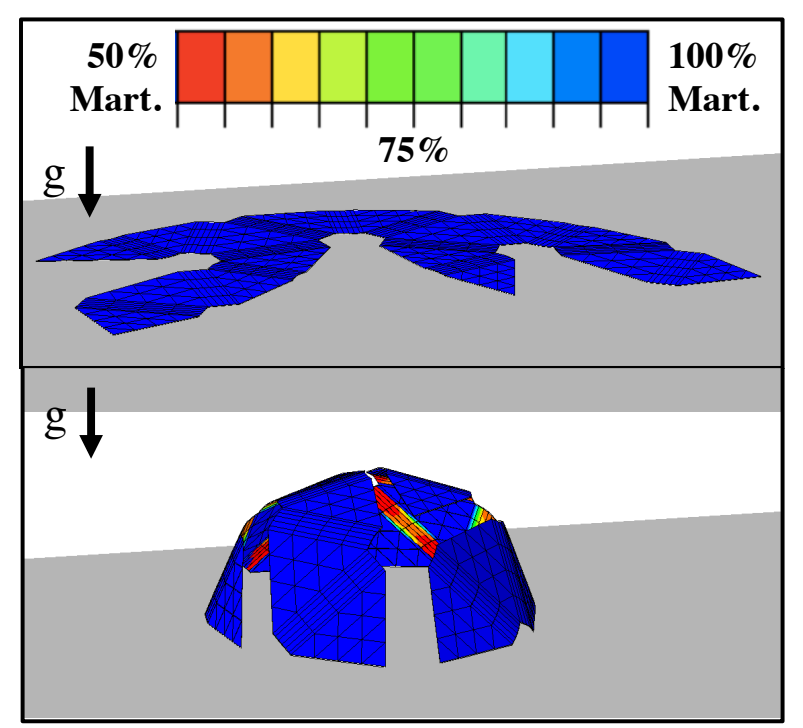

Fig. 20: Initial and final configuration of a reconfigurable SMA composite. Selected heating of prestrained wires from the initial configuration is used to selectively transform the material and induce the deformations necessary to produce the final shape. Reprinted from [351] with permission from Elsevier.

laminated composite beam. According to their results, the recovery stress of the SMA reinforcement and constrained recovery can improve stability of vibration behavior.

Design - The FEM has also been used to analyze SMA-based self-folding laminated composites [349-351]. The self-folding systems in such works consist of a three-layer laminated plate with two outer layers composed of either pre-strained dense SMA films or pre-strained SMA wire meshes. The middle layer is a compliant, thermally insulating elastomer. When either side of the laminate is heated in a line-like region up to a certain SMA austenitic transformation temperature, the heated SMA layer begins recovering the pre-strains and contracts, generating a fold at the heated line in the direction of the side being heated. An example of such a process is shown in Fig. 20. In addition, Peraza Hernandez et al. [349,350] have coupled their detailed FE models with optimization frameworks thereby enabling the design of optimal structural configurations and loading conditions to meet various criteria and shapes. Similarly, Park and coworkers [340,339] considered the problem of a variable twist proprotor for a UAV and studied the performance of a hybrid material consisting of laminated composite embedded with SMA wires. In this approach, a 1D constitutive model was coupled with a lamina-based composite model and used to find an optimal blade configuration to maximize the tip twist [339] and study the flight performance characteristics [340].

\section{Discussion and Perspectives}

As evident from the previous sections, substantial work spanning fabrication, characterization, and modeling has been performed in recent decades in the area of SMA composites. The previous sections delve into these areas in detail, but here some key issues are discussed, especially with respect to what remains to be addressed, as well as future research perspectives.

\subsection{Discussion}

\subsubsection{Fabrication}

Despite the recent developments in powder metallurgy, infiltration, curing techniques, and ultrasonic methods related to SMA composites, many fabrication issues have not yet been satisfactorily ad- 
dressed. For example, residual stresses and load transfer induced by the large deformations associated with martensitic transformation and TRIP remain an issue. In early efforts SMA wires were inserted into sleeves to isolate the matrix from the wires and their associated incompatibilities. Subsequent efforts generally moved away from such a configuration to avoid the additional complication and its limitations. Over the years, the load transfer of various reinforcement types have been experimentally characterized and the interfacial strength between SMAs and a number of matrices has been measured. These issues are especially important with respect to the fatigue and fracture of composite specimens and the performance of these interfaces over repeated cycles has not yet been addressed. In metal and ceramic matrix systems, this problem is compounded by the presence of reaction/interphase layers between the SMA and matrix material that have often been noted. The formation of these layers is a result of species diffusion between the various phases and produces a concentration gradient. Due to the strong compositional sensitivity of SMAs, these layers lead to variations in the constitutive response of the local SMA phases and even additional non-transforming material. The constitutive behavior of these interphases and their ability to withstand the aforementioned large kinematic incompatibilities is expected to strongly influence the interface strength and corresponding composite behaviors. A number of efforts have been performed considering various changes in the processing or pre-treatment of these composites to minimize (or eliminate) these reaction layers and address this issue, however, a comprehensive understanding across the spectra of possible matrix materials and reinforcement types is not yet apparent. The recent growth in interest in various additive manufacturing techniques $(e . g$. $3 \mathrm{D}$ printing) poses both additional possibilities and challenges. Like some of the efforts listed herein, near net-shape manufacturing holds exciting promise for control of the microstructure enabling optimized, tailored material to meet application based demands. The impact of such methodologies on the underlying constituent response and uniformity of the phases, however, has yet to be fully assessed. Further investigations into developing such approaches for SMA composites should be explored along with detailed characterization of the underlying texture and phase distribution and the homogeneity of response. Finally, most of the investigations have focused on small-scale, primarily research, applications and the suitability of the fabrication techniques for large-scale commercial production has not been addressed. With respect to polymer-based systems, that would mean considering upscaling fixtures primarily used to pre-strain and constrain the SMA wires or complementary curing techniques. With respect to metal and ceramic matrix composites, given the strong compositional dependence and impact of the reaction layers, the capability of the powder based techniques to produce such composites with repeatable, homogeneous microstructures needs to be considered and sensitivities of the response to various processing conditions determined.

\subsubsection{Characterization}

The wide variety of SMA composites encompass a broad range of properties, constituents, and responses whose characterization has been proven to be a very complex task. Added to the inherent difficulties in characterizing these materials is the limited commonality in terms of definitions, methodologies, and specific terminology thereby hindering communication and progress. Recently, collaborative efforts across a number of groups (via the CASMART organization) have lead to some design practices and common terms and methodologies with respect to bulk SMA actuators [46]. Given the diversity of measurements (e.g., tangent vs. secant modulus determination), temperatures, conditions (pre-strain), and materials considered, a similar effort is needed for SMA composites to aid in their adoption in applications. Along a similar vein, as the spectrum of SMA composite material systems has grown, so has the number of parameters. The corresponding experimental studies have performed a number of parametric studies, but the complete space is far from being covered. Additionally, in most cases, only a limited number of experiments have been performed. Larger numbers of repeated experiments are necessary, however, to properly characterize the variability of the material properties and their sensitivity. From a different perspective, SMA composites comprise a wide set of linear and nonlinear thermomechanical constitutive responses. The impact of the various deformation mechanisms active, whether transformation, plasticity, TRIP, or twinning effects in the SMA phase or additional inelastic mechanisms in the matrix phase, on the effective composite response has not been fully explored. Experimentally, distinguishing these various effects at a macroscopic level is quite difficult and requires additional data, modeling, or detailed microscopic efforts. Novel approaches (likely through various combined in situ methods) are necessary to address this entanglement. Finally, recent efforts with 
high-temperature SMAs with multiple inelastic mechanisms (transformation, plasticity, and viscoplasticity) have focused on the development of deformation mechanism maps [14] to identify the operative response at different stress-temperature conditions [231]. With the different inelastic mechanisms that are observed in some of these composites, the development of such maps (potentially in conjunction with material-property maps) could be proved to be quite beneficial in subsequent material design and application.

\subsubsection{Modeling}

Some relative strengths and weaknesses of the different approaches undertaken towards modeling the response of SMA composites are discussed in this section in an effort to aid subsequent modeling efforts. The "classical" mean-field approaches based on the Eshelby solution treat only the phase average response of constituents thereby greatly simplifying the considered computational problem at the expense of capturing some heterogeneity-driven, local effects. For example, these approaches lead to a stiffer response during transformation, which in turn affects the prediction of the effective composite transformation characteristics like transformation strain or completion stress/temperature. The magnitude of this overestimation in response depends heavily on the stiffness tensor used (secant, isotropic tangent, or anisotropic tangent) and can be quite substantial. Additional complications arise from inelasticity in the matrix as the stress fields are non-uniform and local effects like yielding may be incorrectly predicted. One other SMA specific issue may be the thermal strain and stress concentration tensors. Previous investigations have focused mainly on issues associated with CTE mismatch related effects and homogeneous temperature fields in the composites. For SMA-based constituents, larger, thermally driven SME related strains may be observed and should be accounted for, but more importantly the strong thermomechanical coupling in the SMA response needs to be addressed. Specifically, depending on the thermal transport characteristics of the composite (e.g., matrix and/or interface thermal conductivity, reinforcement size and dispersion), the latent heat of transformation may lead to apparent hardening of the SMA constituent phase that needs to be accounted for in the constitutive response. Nonetheless, although care needs to be taken in the utilization and formulation of these models, mean-field approaches remain a potent tool for the investigation of SMA composites - especially for design and optimization purposes due their computationally inexpensive nature. Many of the aforementioned deficiencies of the mean-field approaches may be overcome with full-field techniques. Regular arrays of inclusions or locally random microstructures may be modeled by UCs containing the smallest number of inhomogeneities required to replicate the entire array or correctly mimic the local microstructural statistics. More complex microstructures (e.g., bimodal porosity), however, are liable to need an RVE treatment. Such complex RVEs, however, must contain sufficient numbers of inhomogeneities in order to achieve the required homogeneous displacement-traction boundary condition equivalence. Additionally, the use of an SMA phase may introduce additional scales which must be considered in the appropriate selection of the RVE. For example, depending on the reinforcement size, scales associated with the thermal transport between SMA constituents and twinning behavior of the material may need to be considered in some instances. However, in constructing these RVEs, there is a trade-off between accuracy and computational cost that needs to be considered.

\subsection{Perspectives}

\subsubsection{Microstructure Sensitive Analysis and Homogenization}

Increasingly, improvements in manufacturing processes are enabling greater control of microstructures in terms of shape, size, and even distribution of the various reinforcement phases. Additionally, complex and detailed microstructural characterization techniques (e.g, X-ray microtomography and electron backscatter diffraction), which are more frequently used, enable the determination of complex phase distributions, e.g., the identification of interface and reaction layers and corresponding composition gradients. These techniques are capable of producing tremendous sets of data and until recently the incorporation of such detail was computationally intractable. A number of efforts, however, have been undertaken to develop both data processing, reduction, and sampling techniques [256] that can develop usable computational domains of such problems. Statistical representations [294] 
(via parameters like $n$-point correlation functions [424] and statistical volume elements (SVEs) [369]) have also been used to parameterize the datasets and produce equivalent RVEs. For instance, imagebased modeling using microtomography and/or serial-sectioning $[255,368]$ has been extensively used in studying the elastic-plastic behavior of polycrystalline metals [368,158,159]. With respect to SMAs, additional complexity may be found associated with the phase change characteristic of these materials. Specifically, the underlying microstructure becomes temperature dependent thereby requiring that the previously mentioned techniques have an in situ thermal source to characterize both the martensitic and austenitic configurations. Such an approach is necessary both to determine the difference (if any) between the two states and, as the austenitic state is typically taken as the reference state in SMA models, determine the appropriate initial microstructure. Additionally, the martensitic transformation of the SMA phase introduces additional length scales in terms of variant length and precipitate size (amongst others). Multiscale characterization approaches are needed to quantify these parameters in the composite and bring the information up to the mesoscale analysis to incorporate related effects. Bearing this in mind, these increasingly powerful tools represent a key way to produce detailed RVEs capable of analyzing the impact of multiple phases and heterogeneous phase distributions on both local and, more importantly, global responses via homogenization techniques. Analysis coupling such effects can provide key insights into the coupling between microstructural features and phase transformation behaviors (e.g, twinning) providing essential feedback to the material development community.

\subsubsection{Inverse Property Determination and Localization}

One key issue for the numerical analysis of composites and their structures, regardless of complexity, is the determination of the properties of the individual phases. Typically, such measurements are done separately on bulk specimens produced via similar, albeit different, production methods. This approach, however, neglects to account for any effect of the composite processing route (e.g., different diffusional species, variations in applied stress, etc.) on the sample production. Given the sensitivity of SMAs (especially NiTi) to subtle variations in processing and composition, even small differences in this regard could lead to pronounced differences in the properties between the composite and bulk comparison materials - and therefore model results. To address this issue, researchers have started using inverse methodologies for parameter identification and characterization based on high fidelity modeling and full-field characterization techniques (especially DIC). Such efforts combine the modeling and experiments, in a coupled, iterative fashion, to determine the properties of the different phases. Parameter identification of bulk SMA materials has been recently attempted for homogeneous loading experiments, utilizing a gradient-based inverse approach $[297,450]$. In the composite case, however, a number of additional challenges need to be addressed to increase the adoption of such approaches. The first, and potentially most challenging issue, is the determination and characterization of appropriate computational and experimental domains. As the previous sections have illustrated, characteristic sizes of the various phases encompass a wide spectrum - from millimeters to microns - and additional phases like porosity and reaction/interface layers may be present. Experimentally, full-field characterization of these smaller length scales can be quite complex and would likely require microscopic loadings in SEMs or voxel-scale Digital Volume Correlations [40,245]. Even with only two phases, clear identification of the different constituents becomes a critical issue and the presence of additional phases (whose constitutive behaviors may be unknown) only complicates that problem. Furthermore, in actuation cases, controllable temperature stages are required and could provide yet another problem that needs to be addressed. Numerically, the statistically representative nature of the constituents would have to be assessed and careful consideration of appropriate boundary conditions would be necessary to match experimental conditions. Nonetheless, however difficult, the development of the coupled modeling and experimental framework capable of addressing these challenges provides a powerful tool for the composite analysis. Additionally, as a byproduct, the impact of the manufacturing conditions on the local constitutive properties could be investigated and used in subsequent process development.

\subsubsection{Predictive modeling under uncertainty for design}

Another critical issue that needs to be addressed is material variability across manufacturing and processing lots. Often, this issue manifests as differences in SMA component behavior or wide tolerances on admissible material properties, both of which are problematic for most commercial applications. 
Newer techniques investigating the uncertainty inherit in such applications can help bring this issue under control. To date, however, limited efforts may be found addressing this problem for SMA structures $[321,105,106]$. More substantial efforts have been developed for use with the analysis of composite materials both via micromechanical [89] and statistical FE approaches [393] - although most efforts have been with elastic constituents. With respect to SMA composites, this problem becomes only more challenging. Specifically, the parameter space expands from structural design and SMA constitutive parameters to include microstructural features (e.g., inhomogeneity dimensions and concentration) and the material parameters (and response) of the matrix/inhomogeneity phase(s) which would readily vary with deviations in the processing route. Additionally, given the history dependent constitutive responses, variations in loading path may also need to be introduced adding another dimension to the analysis. Experimental determination and quantification of the statistical sensitivity of such materials (and future SMA composites) would prove to be expensive (in terms of cost and time) and tedious. Nonetheless, for commercial viability and increased utilization addressing such concerns is a challenge that must be met. Therefore, a flexible framework for analyzing these material systems and determining bounds on their response should be developed. Tackling this problem will likely incorporate the development of statistical micromechanical models (like that used for studying polycrystalline SMAs [55]) and finite element-based numerical design and optimization techniques used for structural and origami analysis [321,350]. Furthermore, efficient combination of the averaging schemes and detailed approaches discussed in Section 5.2.1 would further enable investigation of both global and local effects.

Acknowledgements This work was supported by AFOSR under MURI award number FA9550-09-1-0686 and the authors would like to thank the program manager Dr. David Stargel. Additional support has been provided by the NSF International Institute of Multifunctional Materials for Energy Conversion (IIMEC) (award number 0841082). B. T. Lester gratefully acknowledges the support of the Sandia National Laboratory-Texas A\&M University Excellence in Engineering fellowship program and the past support of the NSF IGERT program.

\section{References}

1. Aboudi, J.: The generalized method of cells and high-fidelity generalized method of cells micromechanical models - A review. Mechanics of Advanced Materials and Structures 11, 329-366 (2004)

2. Alebrahim, R., Haris, S., Mohamed, N., Abdullah, S.: Vibration analysis of self-healing hybrid composite beam under moving mass. Composite Structures 119, 463-476 (2015)

3. Alecci, V., Bati, S.B., Ranocchiai, G.: Numerical homogenization techniques for the evaluation of mechanical behavior of a composite with SMA inclusions. Journal of the Mechanics of Materials and Structures 4(10), 1675-1688 (2009)

4. Anderson, A., Pedersen, D., Sivertsen, A., Sangesland, S.: Detailed study of shape memory alloys in oil well applications. SINTEF Petroleum Research, Trondheim, Norway (1999)

5. Angioni, S.L., Meo, M., Foreman, A.: Impact damage resistance and damage suppression properties of shape memory alloys in hybrid composites - A review. Smart Materials and Structures 20 (2011). Art. no. 013001

6. Antico, F.C., Zavattieri, P.D., Hector Jr, L.G., Mance, A., Rodgers, W.R., Okonski, D.A.: Adhesion of nickel-titanium shape memory alloy wires to thermoplastic materials: theory and experiments. Smart Materials and Structures 21 (2012). Art. no 035022

7. de Araújo, C.J., Rodrigues, L.F.A., Coutinho Neto, J.F., Reis, R.P.B.: Fabrication and static characterization of carbon-fiber-reinforced polymers with embedded NiTi shape memory wire actuators. Smart Materials and Structures 17 (2008). Art. no. 065004

8. Armstrong, W.D., Kino, H.: Martensitic transformations in a NiTi fiber reinforced 6061 aluminum matrix composite. Journal of Intelligent Material Systems and Structures 6, 809-816 (1995)

9. Arnaboldi, S., Bassani, P., Biffi, C.A., Tuissi, A., Carnevale, M., Lecis, N., LoConte, A., Previtali, B.: Simulated and experimental damping properties of a SMA/Fiber glass laminated composite. Journal of Materials Engineering and Performance 20(4-5), 551-558 (2011)

10. Arnaboldi, S., Bassani, P., Passaretti, F., Redaelli, A., Tuissi, A.: Fabrication and static characterization of carbon-fiber-reinforced polymers with embedded NiTi shape memory wire actuators. Journal of Materials Engineering and Performance 20(4-5), 544-550 (2011)

11. Asadi, H., Akbarzadeh, A., Wang, Q.: Nonlinear thermo-inertial instability of functionally graded shape memory alloy sandwich plates. Composite Structures 120, 496-508 (2015)

12. Asadi, H., Bodaghi, M., Shakeri, M., Aghdam, M.M.: Nonlinear dynamics of SMA-fiber-reinforced composite beams subjected to a primary/secondary-resonance excitation. Acta Mechanica pp. 1-19 (2014). DOI 10.1007/s00707-014-1191-4 
13. Asadi, H., Kiani, Y., Shakeri, M., Eslami, M.R.: Exact solution for nonlinear thermal stability of hybrid laminated composite timoshenko beams reinforced with SMA fibers. Composite Structures 108, 811-822 (2014)

14. Ashby, M.F.: A first report on deformation-mechanism maps. Acta Metallurgica 20, 887-897 (1972)

15. Ashby, M.F.: Overview no. 80: On the engineering properties of materials. Acta Metallurgica 37(5), 1273-1293 (1989)

16. Ashby, M.F., Bréchet, Y.J.M.: Designing hybrid materials. Acta Materialia 53, 5801-5821 (2003)

17. Ashrafi, M.J., Arghavani, J., Naghdabadi, R., Sohrabpour, S.: A 3-D constitutive model for pressuredependent phase transformation of porous shape memory alloys. Journal of the Mechanical Behavior of Biomedical Materials 42, 292-310 (2015)

18. Auricchio, F., Petrini, L.: A three-dimensional model describing stress-temperature induced solid phase transformations: thermomechanical coupling and hybrid composite applications. International Journal for Numerical Methods in Engineering 61, 716-737 (2004)

19. Auricchio, F., Taylor, R.L., Lubliner, J.: Shape-memory alloys: Macromodelling and numerical simulations of the superelastic behavior. Computer Methods in Applied Mechanics and Engineering 146, 281-312 (1997)

20. Aurrekoetxea, J., Zurbitu, J., Ortiz de Mendibil, I., Agirregomezkorta, A., Sánchez-Soto, M., Sarrionandia, M.: Effect of super elastic shape memory alloy wires on the impact behavior of carbon fiber reinforced in situ polymerized poly(butylene terephthalate) composites. Materials Letters 65, 863-865 (2011)

21. Aydogmus, T.: Processing of interpenetrating Mg-TiNi composites by spark plasma sintering. Materials Science \& Engineering A 624, 261-270 (2015)

22. Aydoğmus, T., Bor, d.: Enhanced sintering of TiNi shape memory foams under Mg vapor atmosphere. Metallurgical and Material Transactions A 43A, 5173-5181 (2012)

23. Aydoğmus, T., Bor, d.: Superplasticity and compression behavior of porous TiNi alloys produced using $\mathrm{Mg}$ spacers. Journal of the Mechanical Behavior of Biomedical Materials 15, 59-69 (2012)

24. Aydoğmus, T., Bor, E.T., Bor, d.: Phase transformation behavior of porous TiNi alloys produced by powder metallurgy using magnesium as a space holder. Metallurgical and Material Transactions A 42A, 2547-2555 (2011)

25. Balapgol, B.S., Bajoria, K.M., Kulkarni, S.A.: A two-dimensional finite element analysis of a shape memory alloy laminated composite plate. Smart Materials and Structures 15, 1009-1020 (2006)

26. Bansiddhi, A., Dunand, D.C.: Shape memory NiTi foams produced by solid-state replication with NaF Intermetallics 15, 1612-1622 (2007)

27. Bansiddhi, A., Dunand, D.C.: Shape-memory NiTi foams produced by replication of NaCl space-holders. Acta Biomaterialia 4, 1996-2007 (2008)

28. Bansiddhi, A., Dunand, D.C.: Shape-memory NiTi-Nb foams. Journal of Materials Research 24(6), 2007$2117(2009)$

29. Bansiddhi, A., Dunand, D.C.: Niobium wires as space holder and sintering aid for porous NiTi. Advanced Engineering Materials 13(4), 301-305 (2011). DOI 10.1002/adem.201000241

30. Bansiddhi, A., Dunand, D.C.: Processing of NiTi foams by transient liquid phase sintering. Journal of Materials Engineering and Performance 20(4-5), 511-516 (2011)

31. Bansiddhi, A., Sargeant, T.D., Stupp, S.I., Dunand, D.C.: Porous NiTi for bone implants: A review. Acta Biomaterialia 4, 773-782 (2008)

32. Barrado, M., Lopez, G.A., No, M.L., San Juan, J.: Composites with ultra high damping capacity based on powder metallurgy shape memory alloys. Materials Science \& Engineering A 521-522, 363-367 (2009)

33. Barrett, R., Gross, R.S.: Super-active shape-memory alloy composites. Smart Materials and Structures 5, 255-260 (1996)

34. Barrie, F., Futch, D.B., Hsu, D.H.D., Manuel, M.V.: Effect of phase on debunk strength in shape memory alloy reinforced composites. Materials and Design 57, 98-102 (2014)

35. Barsoum, M.W.: The $M_{N+1} A X_{N}$ phases: A new class of solids; thermodynamically stable nanolaminates. Progress in Solid State Chemistry 28, 201-281 (2000)

36. Barsoum, M.W., Radovic, M.: Elastic and mechanical properties of MAX phases. Annual Review of Materials Research 41, 195-227 (2011)

37. Basu, S., Obando, N., Gowdy, A., Karaman, I., Radovic, M.: Long-term oxidation of $\mathrm{Ti}_{2} \mathrm{AlC}$ in air and water vapor at $1000-1300^{\circ} \mathrm{C}$ temperature range. Journal of the Electrochemical Society 159(2), C90-C96 (2012)

38. Basu, S., Ozaydin, M.F., Kothalkar, A., Karaman, I., Radovic, M.: Phase and morphology evolution in high-temperature $\mathrm{Ti}_{3} \mathrm{SiC}_{2}$-NiTi diffusion-bonded joints. Scripta Materialia 65, 237-240 (2011)

39. Baxevanis, T., Cox, A., Lagodas, D.C.: Micromechanics of precipitated near-equiatomic Ni-rich NiTi shape memory alloys. Acta Mechanica 225, 1167-1185 (2014)

40. Bay, B., Smith, T., Fyhrie, D., Saad, M.: Digital volume correlation: Three-dimensional strain mapping using x-ray tomography. Experimental Mechanics 39, 217-226 (1999)

41. Baz, A., Poh, S., Ro, J., Gilheany, J.: Control of the natural frequencies of NiTiNOL-reinforced composite beams. Journal of Sound and Vibration 185(1), 171-185 (1995)

42. Baz, A., Ro, J.: Thermo-dynamic characteristics of NITINOL reinforced composite beams. Composites Engineering 2(5-7), 527-542 (1992)

43. Baz, A., Ro, J.: Torsional stiffness of NiTiNOL-reinforced composite drive shafts. Composites Engineering 3(12), 1119-1130 (1993)

44. Baz, A., Ro, J.: Optimal vibration control of NiTiNOL-reinforced composites. Composites Engineering 4(6), 567-576 (1994) 
45. Bellouard, Y.: Shape memory alloys for microsystems: A review from a material perspective. Materials Science \& Engineering A 481-482, 582-589 (2008)

46. Benefan, O., Brown, J., Calkins, F.T., Kumar, P., Stebner, A.P., Turner, T.L., Vaidyanathan, R., Webster, J., Young, M.L.: Shape memory alloy actuator design: CASMART collaborative best practices and case studies. International Journal of Mechanics and Materials in Design 10, 1-42 (2014)

47. Benveniste, Y.: A new approach to the application of Mori-Tanaka's theory in composite materials. Mechanics of Materials 6, 147-157 (1987)

48. Benveniste, Y., Dvorak, G.: On a correspondence between mechanical and thermal effects in two-phase composites. In: G. Weng, M. Taya, H. Abé (eds.) Micromechanics and Inhomogeneity, pp. 65-81. Springer New York (1990)

49. Berman, J.B., White, S.R.: Theoretical modeling of residual and transformational stresses in SMA composites. Smart Materials and Structures 5, 731-743 (1996)

50. Bernard, S., Balla, V.K., Bose, S., Bandyopadhyay, A.: Rotating bending fatigue response of laser processed porous NiTi alloy. Materials Science and Engineering C 31, 815-820 (2011)

51. Bernard, S., Balla, V.K., Bose, S., Bandyopadhyay, A.: Compression fatigue behavior of laser processed porous NiTi alloy. Journal of the Mechanical Behavior of Biomedical Materials 13, 62-68 (2012)

52. Bertolino, G., Arneodo Larochette, P., Castrodeza, E.M., Mapelli, C., Baruj, A., Troiani, H.E.: Mechanical properties of martensitic $\mathrm{Cu}-\mathrm{Zn}-\mathrm{Al}$ foams in the pseudoelastic regime. Materials Letters 64, 1448-1450 (2010)

53. Bertolino, G., Gruttadauria, A., Arneodo Larochette, P., Castrodeza, E.M., Baruj, A., Troiani, H.E. Cyclic pseudoelastic behavior and energy dissipation in as-cast $\mathrm{Cu}-\mathrm{Zn}-\mathrm{Al}$ foams of different densities. Intermetallics 19, 577-585 (2011)

54. Bewerse, C., Brinson, L.C., Dunand, D.C.: NiTi with 3D-interconnected micro channels produced by liquid phase sintering and electrochemical dissolution of steel tubes. Journal of Materials Processing Technology 214, 1895-1899 (2014)

55. Bhattacharyya, A., Lagoudas, D.C.: A stochastic thermodynamic model for the gradual thermal transformation of polycrystals. Smart Materials and Structures 6, 235-250 (1997)

56. Birman, V.: Review of mechanics of shape memory alloy structures. Applied Mechanics Reviews 50(11), 629-645 (1997)

57. Birman, V.: Effect of elastic or shape memory alloy particles on the properties of fiber-reinforced composites. Journal of Mechanics of Materials and Structures 4(7-8), 1209-1225 (2009)

58. Birman, V.: Properties and response of composite material with spheroidal super elastic shape memory alloy inclusions subject to three dimensional stress state. Journal of Physics D: Applied Physics 43 (2010). Art. no. 225402

59. Blaiszik, B.J., Kramer, S.L.B., Olugebefola, S.C., Moore, J.S., Sottos, N.R., White, S.R.: Self-healing polymers and composites. Annual Review of Materials Research 40, 179-211 (2010)

60. de Blonk, B., Lagoudas, D.: Actuation of elastomeric rods with embedded two-way shape memory alloy actuators. Smart Materials and Structures 7, 771-783 (1998)

61. Bo, Z., Lagoudas, D.C.: Thermomechanical modeling of polycrystalline SMAs under cyclic loading, Part IV: Modeling of minor hysteresis loops. International Journal of Engineering Science 37, 1205-1249 (1999)

62. Bodaghi, M., Shakeri, M., Aghdam, M.: Thermo-mechanical behavior of shape adaptive composite plates with surface-bonded shape memory alloy ribbons. Composite Structures 119, 115-133 (2015)

63. Bollas, D., Pappas, P., Parthenios, J., Galiotis, C.: Stress generation by shape memory alloy wires embedded in polymer composites. Acta Materialia 55, 5489-5499 (2007)

64. Boonyongmaneerat, Y., Chmielus, M., Dunand, D.C., Müllner, P.: Increasing magneto plasticity in polycrystalline Ni-Mn-Ga by reducing internal constraints through porosity. Physical Review Letters 99 (2007). Art. no. 247201

65. Bor, T.C., Warnet, L., Akkerman, R., de Boer, A.: Modeling of stress development during thermal damage healing in fiber-reinforced composite materials containing embedded shape memory alloy wires. Journal of Composite Materials 44(22), 2547-2572 (2010)

66. Bormann, T., Schulz, G., Deyhle, H., Beckmann, F., de Wild, M., Küffer, J., Münch, C., Hoffman, W. Müller, B.: Combining micro computed tomography and three-dimensional registration to evaluate local strains in shape memory scaffolds. Acta Biomaterialia 10, 1024-1034 (2014)

67. Bouvet, C., Calloch, S., Lexcellent, C.: A phenomenological model for pseudoelasticity of shape memory alloys under multiaxial proportional and nonproportional loadings. European Journal of Mechanics A/Solids 23, 37-61 (2004)

68. Boyd, J.G., Lagoudas, D.C.: Thermomechanical response of shape memory composites. Journal of Intelligent Material Systems and Structures 5, 333-346 (1994)

69. Boyd, J.G., Lagoudas, D.C.: A thermodynamical constitutive model for shape memory materials. Part II. The SMA composite material. International Journal of Plasticity 12(7), 843-873 (1996)

70. Bram, M., Köhl, M., Buchkremer, H.P., Stöver, D.: Mechanical properties of highly porous NiTi alloys. Journal of Materials Engineering and Performance 20(4-5), 522-528 (2011)

71. Briggs, J.P., Ponte Castaneda, P.: Variational estimates for the effective response of shape memory alloy actuated fiber composites. Journal of Applied Mechanics 69, 470-480 (2002). DOI 10.1115/1.1464873

72. Buehler, W.J., Gilfrich, J.V., Wiley, R.C.: Effects of low-temperature phase changes on the mechanical properties of alloys near composition TiNi. Journal of Applied Physics 34, 1475 (1963)

73. Burton, D., Gao, X., Brinson, L.: Finite element simulation of a self-healing shape memory alloy composite. Mechanics of Materials 38(5-6), 525-537 (2006)

74. Calkins, F.T., Mabe, J.H.: Shape memory alloy based morphing aerostructures. Journal of Mechanical Design 132, 111,012 (2010) 
75. Casciati, F., Faravelli, L., Fuggini, C.: Cable vibration mitigation by added SMA wires. Acta Mechanica 195, 141-155 (2008)

76. Castaneda, P., Suquet, P.: Nonlinear composites. Advances in applied mechanics 34(998), 171-302 (1998)

77. Castrodeza, E.M., Mapelli, C., Vedani, M., Arnaboldi, S., Bassani, P., Tuissi, A.: Processing of shape memory CuZnAl open-cell foam by molten metal infiltration. Journal of Materials Engineering and Performance 18(5-6), 484-489 (2009)

78. Chaboche, J., Kanouté, P., Roos, A.: On the capabilities of mean-field approaches for the description of plasticity in metal matrix composites. International Journal of Plasticity 21, 1409-1434 (2005)

79. Chaboche, J., Kruch, S., Maire, J.F., Pottier, T.: Towards a micromechanics based inelastic and damage modeling of composites. International Journal of Plasticity 17, 411-439 (2001)

80. Chang, S.H., Lee, C.Y.: Damping characteristics of TiNi shape memory alloy wires reinforced epoxy resin. Journal of Reinforced Plastics and Composites 30(23), 1931-1938 (2011)

81. Chatterjee, S., Velankar, S.S.: Reversibly texturing active surfaces with spatial and temporal control. Journal of Intelligent Material Systems and Structures 26(3), 328-339 (2015)

82. Chatzigeorgiou, G., Chemisky, Y., Meraghni, F.: Computational micro to macro transitions for shape memory alloy composites using periodic homogenization. Smart Materials and Structures 24 (2015). Art. no. 035009

83. Chaudhry, Z., Rogers, C.A.: Response of composite beams to an internal actuator force. Journal of Mechanical Design 114, 343-348 (1992)

84. Chaudhury, Z., Hailat, M., Liu, Y., Newaz, G.: Aluminum-based composites reinforced with SiC particles and NiTi fibers: influence of fiber dimensions and aging time on mechanical properties. Journal of Materials Science 46, 1945-1955 (2011)

85. Chemisky, Y., Chatzigeorgiou, G., Kumar, P., Lagoudas, D.C.: A constitutive model for cyclic actuation of high-temperature shape memory alloys. Mechanics of Materials 68, 120-136 (2014)

86. Chemisky, Y., Cuval, A., Patoor, E., Ben Zineb, T.: Constitutive model for shape memory alloys including phase transformation, martensitic reorientation and twins accommodation. Mechanics of Materials 43(7), $361-376(2011)$

87. Chemisky, Y., Duval, A., Piotrowski, B., Ben Zineb, T., Tahiri, V., Patoor, E.: Numerical tool for SMA material simulation: Application to composite structure design. Smart Materials and Structures 18(104012) (2009). DOI 10.1088/0964-1726/18/10/104012

88. Chen, G., Cao, P., Edmonds, N.: Porous NiT alloys produced by press-and-sinter from Ni/Ti and $\mathrm{Ni} / \mathrm{TiH}_{2}$ mixtures. Materials Science \& Engineering A 582, 117-125 (2013)

89. Chen, Q., Zhu, H.H., Ju, J.W., Guo, F., Wang, L.B., Yan, Z.G., Deng, T., Zhou, S.: A stochastic micromechanical model for multiphase composites containing spherical inhomogeneities. Acta Mechanica p. In Press

90. Chen, S.Y., Wang, D.H., Han, Z.D., Zhang, C.L., Du, Y.W., Huang, Z.G.: Converse magnetoelectric effect in ferromagnetic shape memory alloy/piezoelectric laminate. Applied Physics Letters 95, 022,501 (2009)

91. Chen, S.Y., Ye, Q.Y., Miao, W., Wang, D.H., Wan, J.G., Liu, J.M., Du, Y.W., Huang, Z.G., Zhou, S.Q.: Direct and converse magnetoelectric effects in $\mathrm{Ni}_{43} \mathrm{Mn}_{41} \mathrm{Co}_{5} \mathrm{Sn}_{11} / \mathrm{Pb}(\mathrm{Zr}, \mathrm{Ti}) \mathrm{O}_{3}$ laminate. Journal of Applied Physics 107, 09D901 (2010)

92. Chen, S.Y., Zheng, Y.X., Ye, Q.Y., Xuan, H.C., Cau, Q.Q., Deng, Y., Wang, D.H., Du, Y.W., Huang, Z.G.: Electric field-modulated hall resistivity and magnetization in magnetoelectric Ni-Mn-Co-Sn/PMNPT laminate. Journal of Alloys and Compounds 509, 8885-8887 (2011)

93. Cheng, F., Hu, L., Reddy, J.N., Karaman, I., Hoffman, E., Radovic, M.: Temperature-dependent thermal properties of a shape memory alloy/MAX phase composite: Experiments and modeling. Acta Materialia 68, 267-278 (2014)

94. Cherkaoui, M., Sun, Q.P., Song, G.Q.: Micromechanics modeling of composite with ductile matrix and shape memory alloy reinforcement. International Journal of Solids and Structures 37(11), $1577-1594$ (2000)

95. Chmielus, M., Witherspoon, C., Wimpory, R.C., Paulke, A., Hilger, A., Zhang, X., Dunand, D.C., Müllner, P.: Magnetic-field-induced recovery strain in polycrystalline Ni-Mn-Ga foam. Journal of Applied Physics 108, 123,526 (2010). DOI 10.1063/1.3524503

96. Chmielus, M., Zhang, X.X., Witherspoon, C., Dunand, D.C., Müllner, P.: Giant magnetic-field-induced strains in polycrystalline Ni-Mn-Ga foams. Nature Materials 8, 863-866 (2009). DOI 10.1038/NMAT2527

97. Cho, H.K., Rhee, J.: Nonlinear finite element analysis of shape memory alloy (SMA) wire reinforced hybrid laminate composite shells. International Journal of Non-Linear Mechanics 47, 672-678 (2012)

98. Collard, C., Ben Zineb, T.: Simulation of the effect of elastic precipitates in SMA materials based on a micromechanical model. Composites: Part B 43, 2560-2576 (2012)

99. Collard, C., Ben Zineb, T., Patoor, E., Salah, M.O.B.: Micromechanical analysis of precipitate effects on shape memory alloys behavior. Materials Science \& Engineering A 481-482, 366-370 (2008)

100. Coppola, A.M., Hu, L., Thakre, P.R., Radovic, M., Karaman, I., Sottos, N.R., White, S.R.: Active cooling of a bilayer shape memory alloy-polymer matrix composite hybrid containing micro channels fabricated by vaporization of sacrificial components. Advanced Functional Materials Under Review (2015)

101. Cortes, P., Terzak, J., Tubas, G., Phillips, D., Baur, J.W.: The morphing properties of a vascular shape memory composite. Smart Materials and Structures 23 (2014). Art. no. 015018

102. Coughlin, J.P., Williams, J.J., Chawla, N.: Mechanical behavior of NiTi shape memory alloy fiber reinforced Sn matrix "smart" composites. Journal of Materials Science 44, 1267-1272 (2009). DOI $10.1007 / \mathrm{s} 10853-008-3188-7$ 
103. Coughlin, J.P., Williams, J.J., Crawford, G.A., Chawla, N.: Interfacial reactions in model NiTi shape memory alloy fiber-reinforced Sn matrix "smart" composites. Metallurgical and Material Transactions A 40A, 176-184 (2009)

104. Cox, A., Franco, B., Baxevanis, T., Karaman, I., Lagoudas, D.: Predictive constitutive modeling of precipitated Ni-rich NiTi SMAs. Submitted

105. Crews, J.H., McMahan, J.A., Smith, R.C., Hannen, J.C.: Quantification of parameter uncertainty for robust control of shape memory alloy bending actuators. Smart Materials and Structures 22 (2013). Art. no. 115021

106. Crews, J.H., Smith, R.C.: Quantification of parameter and model uncertainty for shape memory alloy bending actuators. Journal of Intelligent Material Systems and Structures 25(2), 229-245 (2014)

107. Daghia, F., Inman, D., Ubertini, F., Viola, E.: Shape memory alloy hybrid composite plates for shape and stiffness control. Journal of Intelligent Material Systems and Structures 19, 609-619 (2008)

108. Daly, M., Pequegnat, A., Zhou, Y., Khan, M.I.: Enhanced thermomechanical functionality of a laser processed hybrid NiTiNiTiCu shape memory alloy. Smart Materials and Structures 21 (2012). DOI $10.1088 / 0964-1726 / 21 / 4 / 045018$

109. Damanpack, A.R., Aghdam, M.M., Shakeri, M.: Micromechanics of shape memory alloy fiber-reinforced composites subjected to multi-axial non-proportional loadings. Journal of Intelligent Material Systems and Structures p. In Press (2014). DOI 10.1177/1045389X14556165

110. Damanpack, A.R., Aghdam, M.M., Shakeri, M.: Micro-mechanics of composite with SMA fibers embedded in metallic/polymeric matrix under off-axial loadings. European Journal of Mechanics A/Solids 49, 467$480(2015)$

111. Davis, B., Turner, T.L., Seelecke, S.: Measurement and prediction of the thermomechanical response of shape memory alloy hybrid composite beams. Journal of Intelligent Material Systems and Structures 19, 129-143 (2008)

112. Dixit, M., Newkirk, J.W., Mishra, R.S.: Properties of friction stir-processed AL 1100-NiTi composite. Scripta Materialia 56, 34-52 (2007)

113. Doghri, I., Adam, L., Bilger, N.: Mean-field homogenization of elasto-viscoplastic composites based on a general incrementally affine linearization method. International Journal of Plasticity 26, 219-238 (2010)

114. Doghri, I., Brassart, L., Adam, L., Gérard, J.S.: A second-moment incremental formulation for the meanfield homogenization of elasto-plastic composites. International Journal of Plasticity 27, 352-371 (2011)

115. Dong, J., Cai, C.S., Okeil, A.M.: Overview of potential and existing applications of shape memory alloys in bridges. Journal of Bridge Engineering 16, 305-315 (2011)

116. Drago, A., Pindera, M.J.: Micro-macromechanical analysis of heterogeneous materials: Macroscopically homogeneous vs periodic microstructures. Composites Science and Technology 67(6), 1243-1263 (2007)

117. Drugan, W.J., Willis, J.R.: A micromechanics-based nonlocal constitutive equation and estimates of representative volume element size for elastic composites. J. Mech. Phys. Solids 44(4), 497-524 (1996)

118. Duerig, T., Pelton, A., Stöckel, D.: An overview of NiTiNOL medical applications. Materials Science \& Engineering A 273-275, 149-160 (1999)

119. Dunand, D.C., Mari, D., Bourke, M.A.M., Roberts, J.A.: NiTi and NiTi-TiC composites: Part IV. neutron diffraction study of twinning and shape-memory recovery. Metallurgical and Material Transactions A 27A, 2820-2836 (1996)

120. Dunand, D.C., Müllner, P.: Size effects on the magnetic actuation in Ni-Mn-Ga shape memory alloys. Advanced Materials 23, 216-232 (2011)

121. Dvorak, G.J.: Thermomechanics of heterogeneous media. Journal of Thermal Stresses 20, 799-817 (1997)

122. Eggeler, G., Hornbogen, E., Yawny, A., Heckmann, A., Wagner, M.: Structural and functional fatigue of NiTi shape memory alloys. Materials Science and Engineering A 378(1), 24-33 (2004)

123. Elahinia, M.H., Hashemi, M., Tabesh, M., Bhaduri, S.B.: Manufacturing and processing of NiTi implants: A review. Progress in Materials Science 57, 911-946 (2012)

124. Entchev, P.B., Lagoudas, D.C.: Modeling porous shape memory alloys using micromechanical averaging techniques. Mechanics of Materials 34(1), 1-24 (2002)

125. Entchev, P.B., Lagoudas, D.C.: Modeling of transformation-induced plasticity and its effect on the behavior of porous shape memory alloys: Part II: Porous SMA response. Mechanics of Materials 36(9), 893-913 (2004)

126. Epps, J., Chandra, R.: Shape memory alloy actuation for active tuning of composite beams. Smart Materials and Structures 6, 251-264 (1997)

127. Esen, Z.: The effect of processing routes on the structure and properties of magnesium-TiNi composites. Materials Science \& Engineering A 558, 632-640 (2012)

128. Eshelby, J.D.: The determination of the elastic field of an ellipsoidal inclusion, and related problems. Proceedings of the Royal Society of London. Series A, Mathematical and Physical Sciences 241(1226), 376-396 (1957)

129. Evirgen, A., Karaman, I., Noebe, R.D., Santamarta, R., Pons, J.: Effect of precipitation on the microstructure and the shape memory response of the $\mathrm{Ni}_{50.3} \mathrm{Ti}_{29.7} \mathrm{Hf}_{20}$ high temperature shape memory alloy. Scripta Materialia 69, 354-357 (2013)

130. Evirgen, A., Karaman, I., Santamarta, R., Pons, J., Noebe, R.D.: Microstructural characterization and superelastic response of a $\mathrm{Ni}_{50.3} \mathrm{Ti}_{29.7} \mathrm{Zr}_{20}$ shape memory alloy. Scripta Materialia 81, 12-15 (2014)

131. Evirgen, A., Karaman, I., Santamarta, R., Pons, J., Noebe, R.D.: Microstructural characterization and shape memory characteristics of the $\mathrm{Ni}_{50.3} \mathrm{Ti}_{34.7} \mathrm{Hf}_{15}$ shape memory alloy. Acta Materialia 83, 48-60 (2015) 
132. Faiella, G., Antonucci, V., Daghia, F., Fascia, S., Giordano, M.: Fabrication and thermo-mechanical characterization of a shape memory alloy hybrid composite. Journal of Intelligent Material Systems and Structures 22, 245-252 (2011)

133. Farvizi, M., Ebadzadeh, T., Vaezi, M.R., Yoon, E.Y., Kim, Y.J., Kim, H.S., Simchi, A.: Microstructural characterization of HIP consolidated NiTi-nano $\mathrm{Al}_{2} \mathrm{O}_{3}$ composites. Journal of Alloys and Compounds 606, 21-26 (2014)

134. Feng, X., Sui, J., Cai, W.: Processing of multi-walled carbon nanotube-reinforced TiNi composites by hot pressed sintering. Journal of Composite Materials 45(15), 1553-1557 (2011). DOI $10.1177 / 0021998310383734$

135. Feng, X., Zhao, L., Mi, X., Li, Y., Xie, H., Yin, X., Gao, B.: Improved shape memory composites combined with TiNi wire and shape memory epoxy. Materials and Design 50, 724-727 (2013)

136. Feuchtwanger, J., Richard, M.L., Tang, Y.J., Berkowitz, A.E., O'Handley, R.C., Allen, S.M.: Large energy absorption in Ni-Mn-Ga/polymer composites. Journal of Applied Physics 97, $10 \mathrm{M} 319$ (2005)

137. Feuchtwanger, J., Richard, M.L., Tang, Y.J., Berkowitz, A.E., O'Handley, R.C., Allen, S.M.: Rearrangement of twin variants in ferromagnetic shape memory alloy-polyurethane composites studied by stroboscopic neutron diffraction. Journal of Physics: Condensed Matter 20 (2008). Art. no. 104247

138. Firstov, G.S., Van Humbeeck, J., Koval, Y.N.: High temperature shape memory alloys problems and prospects. Journal of Intelligent Material Systems and Structures 17, 1041 (2006)

139. Fish, J., Shek, K., Pandheeradi, M., Shephard, M.: Computational plasticity for composite structures based on mathematical homogenization: Theory and practice. Computer Methods in Applied Mechanics and Engineering 148(1-2), 53-73 (1997)

140. Freed, Y., Aboudi, J.: Micromechanical investigation of plasticity-damage coupling of concrete reinforced by shape memory alloy fibers. Smart Materials and Structures 17 (2008). Art. no. 015046

141. Freed, Y., Aboudi, J.: Micromechanical prediction of the two-way shape memory effect in shape memory alloy composites. International Journal of Solids and Structures 46, 1634-1647 (2009)

142. Freed, Y., Aboudi, J.: Thermomechanically coupled micromechanical analysis of shape memory alloy composites undergoing transformation induced plasticity. Journal of Intelligent Material Systems and Structures 20, 23-38 (2009)

143. Freed, Y., Aboudi, J., Gilat, R.: Thermomechanically micromechanical modeling of prestressed concrete reinforced with shape memory alloy fibers. Smart Materials and Structures 16, 717-727 (2007)

144. Freed, Y., Aboudi, J., Gilat, R.: Investigation of shape memory alloy honeycombs by means of a micromechanical analysis. Modeling and Simulation in Materials Science and Engineering 56, 3003-3020 (2008)

145. Fukami-Ushiro, K.L., Dunand, D.C.: NiTi-NiTi-TiC composites: Part III. shape-memory recovery. Metallurgical and Material Transactions A 27A, 193-203 (1996)

146. Fukami-Ushiro, K.L., Mari, D., Dunand, D.C.: NiTi-NiTi-TiC composites: Part II. compressive mechanical properties. Metallurgical and Material Transactions A 27A, 183-191 (1996)

147. Furuya, Y.: Design and material evaluation of shape memory composites. Journal of Intelligent Material Systems and Structures 7, 321-330 (1996)

148. Furuya, Y., Sasaki, A., Taya, M.: Enhanced mechanical properties of TiNi shape memory fiber/ Al matrix composite. Materials Transactions, JIM 34(3), 224-227 (1996)

149. Gao, X., Burton, D., Turner, T.L., Brinson, L.C.: Finite element analysis of adaptive-stiffening and shapecontrol SMA hybrid composites. Journal of Engineering Materials and Technology 128, 285-293 (2006)

150. Gavazzi, A.C., Lagoudas, D.C.: On the numerical evaluation of eshelby's tensor and its application to elastoplastic fibrous composites. Computational Mechanics 7, 13-19 (1990)

151. Ghosh, P., Rao, A., Srinivasa, A.R.: Design of multi-state and smart-bias components using shape memory alloy and shape memory polymer composites. Materials and Design 44, 164-171 (2013)

152. Gillet, Y., Patoor, E., Berveiller, M.: Calculation of pseudoelastic elements using a non-symmetrical thermomechanical transformation criterion and associated rule. Journal of Intelligent Material Systems and Structures 9(5), 366-378 (1998)

153. Glock, S., Spārniņš, E., Leterrier, Y., Michaud, V.: Effect of annealing and silylation on the strength of melt-spun Ni-Mn-Ga fibres and their adhesion to epoxy. International Journal of Adhesion \& Adhesives 55, 89-94 (2014)

154. Glock, S., Zhang, X.X., Kucza, N.J., Müllner, P., Michaud, V.: Structural, physical and damping properties of melt-spun Ni-Mn-Ga wire-epoxy composites. Composites: Part A 63, 68-75 (2014)

155. Gong, S., Li, Z., Xiao, Z., Zheng, F.: Microstructure and property of the composite laminate cladded by explosive welding of CuAlMn shape memory alloy and QBe2. Materials and Design 30, 1404-1408 (2009)

156. González, C., Segurado, J., LLorca, J.: Numerical simulation of elasto-plastic deformation of composites: evolution of stress microfilms and implications for homogenization models. Journal of the Mechanics and Physics of Solids 52, 1573-1593 (2004)

157. Granta: CES 2015 edupack. Website (2015). http://www.grantadesign.com/education/edupack/index.htm

158. Groeber, M., Ghosh, S., Uchic, M.D., Dimiduk, D.M.: A framework for automated analysis and simulation of 3D polycrystalline microstructures. Part 1: Statistical characterization. Acta Materialia 56, 1257-1273 (2008)

159. Groeber, M., Ghosh, S., Uchic, M.D., Dimiduk, D.M.: A framework for automated analysis and simulation of 3D polycrystalline microstructures. Part 2: Synthetic structure generation. Acta Materialia 56, 1274$1287(2008)$

160. Gururaja, S., Taya, M., Kang, Y.S.: Design of ferromagnetic shape memory alloy composite made of Fe and TiNi particles. Journal of Applied Physics 102, 064,910 (2007) 
161. Hahnlen, R., Dapino, M.J.: NiTi-Al interface strength in ultrasonic additive manufacturing composites. Composites: Part B 59, 101-108 (2014)

162. Haldar, K., Lagoudas, D.C., Karaman, I.: Magnetic field-induced martensitic phase transformation in magnetic shape memory alloys: Modeling and experiments. Journal of the Mechanics and Physics of Solids 69, 33-66 (2014)

163. Hamada, K., Lee, J., Mizuuchi, K., Taya, M., Inoue, K.: Thermomechanical behavior of tini shape memory alloy fiber reinforced 6061 aluminum matrix composite. Metallurgical and Materials Transactions A: Physical Metallurgy and Materials Science 29 A(3 A), 1127-1135 (1998)

164. Hamming, L.W., Fan, X.W., Messersmith, P.B., Brinson, L.C.: Mimicking mussel adhesion to improve interfacial properties in composites. Composites Science and Technology 68, 2042-2048 (2008)

165. Hannula, S.P., Aaltio, I., Ge, Y., Lahelin, M., Söderberg, O.: Processing and properties of Ni-Mn-Ga magnetic shape memory alloy based hybrid materials. Current Applied Physics 12, S63-S67 (2012)

166. Hao, S., Cui, L., Jiang, D., Han, X., Ren, Y., Jiang, J., Liu, Y., Liu, Z., Mao, S., Wang, Y., Li, Y., Ren, X., Ding, X., Wang, S., Yu, C., Shi, X., Du, M., Yang, F., Zheng, Y., Zhang, Z., Li, X., Brown, D.E., $\mathrm{Li}$, J.: A transforming metal nano composite with large elastic strain, low modulus, and high strength. Science 339, 1191-1194 (2013)

167. Hao, S., Cui, L., Jiang, D., Yu, C., Jiang, J., Shi, X., Liu, Z., Wang, S., Wang, Y., Brown, D.E., Ren, Y.: Nanostructured Nb reinforced NiTi shape memory alloy composite with high strength and narrow hysteresis. Applied Physics Letters 102 (2013). DOI 10.1063/1.4809954

168. Hao, S., Cui, L., Shao, Y., Jiang, J., Jiang, D., Wang, S., Du, M., Wang, Y., Brown, D.E., Ren, Y.: In situ X-ray diffraction study of deformation behavior in a Fe/NiTi composite. Applied Physics Letters 101 (2012). Art. no. 221904

169. Hartl, D., Lagoudas, D.: Thermomechanical chracterization of shape memory alloy materials. In: D. Lagoudas (ed.) Shape Memory Alloys: Modeling and Engineering Applications, chap. 2, pp. 53-119. Springer-Verlag, New York (2008)

170. Hartl, D., Lagoudas, D., Mabe, J., Calkins, F.: Use of Ni60Ti shape memory alloy for active jet engine chevron application, Part I: Thermomechanical characterization. Smart Materials and Structures 19(1) (2009). Art. no. 015020

171. Hartl, D.J., Chatzigeorgiou, G., Lagoudas, D.C.: Three-dimensional modeling and numerical analysis of rate-dependent irrecoverable deformation in shape memory alloys. International Journal of Plasticity $\mathbf{2 6}$, 1485-1507 (2010)

172. Hartl, D.J., Lagoudas, D.: Aerospace applications of shape memory alloys. Proceedings of the Institution of Mechanical Engineers, Part G: Journal of Aerospace Engineering 221 (Special Issue), 535-552 (2007)

173. Hartl, D.J., Lagoudas, D.: Constitutive modeling and structural analysis considering simultaneous phase transformation and plastic yield in shape memory alloys. Smart Materials and Structures 18(10) (2009). Art. no. 104017

174. Hawkins, L.E., Vick, B., Rogers, C.A.: An investigation of the transient thermal response of a shape memory alloy composite beam. In: Proceedings of the AIAA/ASME/ASCE/AHS/ASC $31^{\text {st }}$ Structures, Structural Dynamics and Materials Conference, vol. 0941, pp. 1972-1978 (1990)

175. Hebda, D.A., Whitlock, M.E., Ditman, J.B., White, S.R.: Manufacturing of adaptive graphite/epoxy structures with embedded Nitionol wires. Journal of Intelligent Material Systems and Structures 6, 220-228 (1995)

176. Heller, L., Vokoun, D., Sittner, P., Finckh, H.: 3D flexible NiTi-braided elastomer composites for smart structure applications. Smart Materials and Structures 21 (2012). DOI 10.1088/0964-1726/21/4/045016

177. Herzog, H., Jacquet, E.: From a shape memory alloys model implementation to a composite behavior. Computational Material Science 39, 365-375 (2007)

178. Hill, R.: Elastic properties of reinforced solids: Some theoretical principles. Journal of the Mechanics and Physics of Solids 13, 357-371 (1963)

179. Hill, R.: A self-consistent mechanics of composite materials. Journal of the Mechanics and Physics of Solids 13(4), 213-222 (1965)

180. Hinz, D., Scheerbaum, N., Gutfleisch, O., Müller, K.H., Schultz, L.: Polyester-bonded textured composites with sing-crystalline shape memory Ni-Mn-Ga particles. Journal of Magnetism and Magnetic Materials 310, 2785-2787 (2007)

181. Ho, M.P., Lau, K.T., Au, H.Y., Dong, Y., Tam, H.Y.: Structural health monitoring of an asymmetrical SMA reinforced composite using embedded FBG sensors. Smart Materials and Structures 22 (2013). Art. no. 125015

182. Hosseini, S.A., Sadrnezhaad, S.K., Ekrami, A.: Phase transformation behavior of porous NiTi alloy fabricated by powder metallurgical method. Materials Science and Engineering C 29, 2203-2207 (2009)

183. Hu, J., Wu, G., Zhang, Q., Gou, H.: Mechanical properties and damping capacity of $\mathrm{SiC}_{p} / \mathrm{TiNi}_{f} / \mathrm{Al}$ composite with different volume fraction of SiC particle. Composites: Part B 66, 400-406 (2014)

184. Hu, J., Wu, G., Zhang, Q., Kang, P., Liu, Y.: Microstructure of multilayer interface in an Al matrix composite reinforced by TiNi fiber. Micron 64, 57-65 (2014)

185. Hu, J., Zhang, Q., Liu, Y., Wu, G.: Phase transformation behaviors of TiNi fibers embedded in an aluminum matrix. Journal of Alloys and Compounds 589, 491-497 (2014)

186. Hu, J., Zhang, Q., Wu, G., Liu, Y., Li, D.: Effect of pre-oxidation of TiNi fibers on the interfacial and mechanical property of $\mathrm{TiNi}_{f} / \mathrm{Al}$ composite. Materials Science \& Engineering A 597, 20-28 (2014)

187. Hu, L., Kothalkar, A., Proust, G., Karaman, I., Radovic, M.: Fabrication and characterization of $\mathrm{NiTi} / \mathrm{Ti}_{3} \mathrm{SiC}_{2}$ and $\mathrm{NiTi} / \mathrm{Ti}_{2} \mathrm{AlC}$ composites. Journal of Alloys and Compounds 610, 635-644 (2014)

188. Huang, M., Brinson, L.C.: A multi variant model for single crystal shape memory alloy behavior. Journal of the Mechanics and Physics of Solids 46(8), 1379-1409 (1998) 
189. Huet, C.: Coupled size and boundary-condition effects in viscoelastic heterogeneous and composite bodies. Mech. Mater. 31(12), 787-829 (1999)

190. Ibrahim, H.H., Tawfik, M., Negm, H.M.: Limit-cycle oscillation of shape memory alloy hybrid composite plates at elevated temperatures. Mechanics of Advanced Materials and Structures 16, 429-441 (2009)

191. Irzhak, A., Koledov, V., Zakharov, D., Lebedev, G., Mashirov, A., Afonina, V., Akatyeva, K., Kalashnikov, V., Sitnikov, N., Tabachkova, N., Shelyakov, A., Shavrov, V.: Development of laminated nano composites on the bases of magnetic and non-magnetic shape memory alloys: Towards new tools for nanotechnology. Journal of Alloys and Compounds 586, S464-S468 (2014)

192. Ismail, M.H., Goodall, R., Davies, H.A., Todd, I.: Formation of microporous NiTi by transient liquid phase sintering of elemental powders. Materials Science \& Engineering C 32, 1480-1485 (2012)

193. Ismail, M.H., Goodall, R., Davies, H.A., Todd, I.: Porous NiTi alloy by metal injection moulding/sintering of element powders: Effect of sintering temperature. Materials Letters 70, 142-145 (2012)

194. Itin, V.I., Gyunter, V.E., Shabalovskaya, S.A., Sachdeva, R.L.C.: Mechanical properties and shape memory of porous nitinol. Materials Characterization 32, 179-187 (1994)

195. Jang, B.K., Kishi, T.: Influence of stacking angle of carbon fibers on fracture behavior of TiNi fiber impregnated CFRP composites. Journal of Alloys and Compounds 49, 208-212 (2006)

196. Jia, J., Rogers, C.A.: Formulation of a mechanical model for composites with embedded SMA actuators. Journal of Mechanical Design - Transactions of the ASME 114, 670-676 (1992)

197. Jiang, D., Cui, L., Zheng, Y., Zhao, X., Li, Y.: Constrained martensitic transformation in an in situ lamella TiNi/NbTi shape memory composite. Materials Science \& Engineering A 515, 131-133 (2009)

198. Jiang, D., Jiang, J., Shi, X., Jiang, X., Jiao, S., Cui, L.: Constrained martensitic transformation in nanocrystalline TiNi/NbTi shape memory composites. Journal of Alloys and Compounds In Press (2011). DOI 10.1016/j.jallcom.2011.10.104

199. Jiang, H.J., Cao, S., Ke, C.B., Ma, X., Zhang, X.P.: Nano-sized SiC particle reinforced NiTi alloy matrix shape memory composite. Materials Letters 100, 74-77 (2013)

200. Jiang, J., Cui, L., Zheng, Y., Jiang, D., Liu, Z., Zhao, K.: Narrow hysteresis behavior of TiNi shape memory alloy constrained by NbTi matrix during incomplete transformation. Materials Science \& Engineering A 536, 33-36 (2012)

201. Jiang, J., Cui, L.S., Zheng, Y.J., Jiang, D.Q., Liu, Z.Y., Zhao, K.: Negative thermal expansion arrest point memory effect in TiNi shape memory alloy and $\mathrm{NbTi} / \mathrm{TiNi}$ composite. Materials Science \& Engineering A 549, 114-117 (2012)

202. Johansen, K., Voggenreiter, H., Eggeler, G.: On the effect of TiC particles on the tensile properties and on the intrinsic two way effect of NiTI shape memory alloys produced by powder metallurgy. Materials Science \& Engineering A 273-275, 410-414 (1999)

203. Jonnalagadda, K., Kline, G.E., Sottos, N.: Local displacements and load transfer in shape memory alloy composites. Experimental Mechanics 37(1), 78-86 (1997)

204. Jonnalagadda, K., Sottos, N., Qidwai, M., Lagoudas, D.: Transformation of embedded shape memory alloy ribbons. Mathematics and controls in Smart Structures 3039, 481-486 (1998)

205. Jonnalagadda, K., Sottos, N.R.: Influence of adhesion on micromechanical behavior of SMA composites. In: Proceedings of the SPIE, vol. 2442, pp. 143-151. San Diego, CA (1995)

206. Jung, B.S., Kong, J.P., Li, N., Kim, Y.M., Kim, M.S., Ahn, S.H., Cho, M.: Numerical simulation and verification of a curved morphing composite structure with embedded shape memory alloy wire actuators. Journal of Intelligent Material Systems and Structures 24(1), 89-98 (2012)

207. Jung, B.S., Kong, J.P., Li, N., Kim, Y.M., Kim, M.S., Ahn, S.H., Cho, M.: Numerical simulation and verification of a curved morphing composite structure with embedded shape memory alloy wire actuators. Journal of Intelligent Material Systems and Structures 24(1), 89-98 (2013)

208. Kang, K.W., Kim, J.K.: Effect of shape memory alloy on impact damage behavior and residual properties of glass/epoxy laminates under low temperature. Composite Structures 88, 455-460 (2009)

209. Kanit, T., Forest, S., Galliet, I., Mounoury, V., Jeulin, D.: Determination of the size of the representative volume element for random composites: Statistical and numerical approach. Int. J. Solids Struct. 40(1314), 3647-3679 (2003)

210. Karaca, H., Karaman, I., Basaran, B., Lagoudas, D., Chumlyakov, Y., Maier, H.: On the stress-assisted magnetic-field-induced phase transformation in $n i_{2} m n g a$ ferromagnetic shape memory alloys. Acta Materialia 55, 4253-4269 (2007)

211. Karaca, H., Karaman, I., Basaran, B., Ren, Y., Chumlyakov, Y., Maier, H.: Magnetic field-induced phase transformation in NiMnCoIn magnetic shape-memory alloys - a new actuation mechanism with large work output. Advanced Functional Materials 19, 983-998 (2009)

212. Karaca, H.E., Karaman, I., Basaran, B., Chumlyakov, Y.I., Maier, H.J.: Magnetic field and stress induced martensite reorientation in NiMnGa ferromagnetic shape memory alloy single crystals. Acta Materialia 54(1), 233-245 (2006)

213. Kauffmann-Weiss, S., Scheerbaum, N., Liu, J., Klauss, H., Schultz, L., Mäder, E., Häßler, R., Heinrich, G., Gutfleisch, O.: Reversible magnetic field induced strain in $\mathrm{Ni}_{2} \mathrm{MnGa-polymer-composites}$. Advanced Engineering Materials 14(1-2), 20-27 (2012). DOI 10.1002/adem.201100128

214. Khalili, S.M.R., Shiravi, M., Nooramin, A.S.: Mechanical behavior of notched plate repaired with polymer composite and smart patches - experimental study. Journal of Reinforced Plastics and Composites 29(19), 3021-3037 (2010). DOI 10.1177/0731684410363179

215. Khalili, S.M.R., Shokuhfar, A., Ashenai Ghasemi, F., Malekzadeh, K.: Dynamic response of smart hybrid composite plate subjected to low-velocity impact. Journal of Composite Materials 41, 2347-2370 (2007)

216. Kiefer, B., Lagoudas, D.C.: Magnetic field-induced martensitic variant reorientation in magnetic shape memory alloys. Philosophical Magazine Special Issue: Recent Advances in Theoretical Mechanics, in Honor of SES 2003 A.C. Eringen Medalist G.A. Maugin 85(33-35), 4289-4329 (2005) 
217. Kiefer, B., Lagoudas, D.C.: Modeling of magnetic SMAs. In: D. Lagoudas (ed.) Shape Memory Alloys: Modeling and Engineering Applications, chap. 7, pp. 53-119. Springer-Verlag, New York (2008)

218. Kim, D.J., Kim, H.A., Chung, Y.S., Choi, E.: Pullout resistance of straight NiTi shape memory alloy fibers in cement mortar after cold drawing and heat treatment. Composites: Part B 67, 588-594 (2014)

219. Kim, D.J., Kim, H.A., Chung, Y.S., Choi, E.: Pullout resistance of deformed shape memory alloy fibers embedded in cement mortar. Journal of Intelligent Material Systems and Structures p. In Press (2015). DOI 10.1177/1045389X14566524

220. Kim, E.H., Lee, I., Roh, J.H., Bae, J.S., Choi, I.H., Koo, K.N.: Effects of shape memory alloys on low velocity impact characteristics of composite plates. Composite Structures 93, 2903-2909 (2011)

221. Kim, H.J., Song, S.H., Ahn, S.H.: A turtle-like swimming robot using a smart soft composite (SSC) structure. Smart Materials and Structures 22, 014,007 (2013)

222. Kirkby, E.L., Michaud, V.J., Månson, J.A.E., Sottos, N.R., White, S.R.: Performance of self-healing epoxy with micro encapsulated healing agent and shape memory alloy wires. Polymer 50, 5533-5538 (2009)

223. Kirkby, E.L., O'Keane, J., de Oliveira, R., Michaud, V.J., Månson, J.A.E.: Tailored processing of epoxy with embedded shape memory alloy wires. Smart Materials and Structures 18 (2009). Art. no. 095043

224. Kirkby, E.L., Rule, J.D., Michaud, V.J., Sottos, N.R., White, S.R., Månson, J.A.E.: Embedded shapememory alloy wires for improved performance of self-healing polymers. Advanced Functional Materials 18, 2253-2260 (2008)

225. Ko, P.L., Chang, F.L., Li, C.H., Chen, J.Z., Cheng, I.C., Tung, Y.C., S.-H., C., Lin, P.C.: Dynamically programmable surface micr-wrinkles on PDMS-SMA composite. Smart Materials and Structures 23 (2014). Art. no. 115007

226. Kockar, B., Karaman, I., Kim, J.I., Chumlyakov, Y.I., Sharp, J., Yu, C.J.: Thermomechanical cyclic response of an ultrafin-grained niti shape memory alloy. Acta Materialia 56, 3630-3646 (2008)

227. Kockar, B., Ozcan, H., Cakmak, S.: Shape memory behavior of Ni-rich NiTi foam with different porosity percentages. Journal of Intelligent Material Systems and Structures 24(9), 1131-1137 (2013). DOI $10.1177 / 1045389$ X12469450

228. Kohlhaas, B., Klinkel, S.: An $\mathrm{FE}^{2}$ model for the analysis of shape memory alloy fiber-composites. Computational Mechanics 55, 421-437 (2015)

229. Kothalkar, A., Cerit, A., Proust, G., Basu, S., Radovic, M., Karaman, I.: Interfacial study of NiTi-Ti $i_{3} \mathrm{SiC}_{2}$ solid state diffusion bonded joints. Materials Science \& Engineering A 622, 168-177 (2015)

230. Kothalkar, A.D., Benitez, R., Hu, L., Radovic, M., Karaman, I.: Thermo-mechanical response and damping behavior of shape memory alloy - MAX phase composites. Metallurgical and Material Transactions A 45A, 2646-2658 (2014). DOI 10.1007/s11661-014-2193-5

231. Kumar, P.K., Caer, C., Atkinson, G., Patoor, E., Lagoudas, D.C.: The influence of stress and temperature on the residual strain generated during pseudoelastic cycling of niti sma wires. In: Proceedings of SPIE, vol. 7978 , pp. 306-312 (2006)

232. Lagoudas, D. (ed.): Shape Memory Alloys: Modeling and Engineering Applications. Springer-Verlag, New York (2008)

233. Lagoudas, D., Hartl, D., Chemisky, Y., Machado, L., Popov, P.: Constitutive model for the numerical analysis of phase transformation in polycrystalline shape memory alloys. International Journal of Plasticity 32-33, 155-183 (2012)

234. Lagoudas, D., Vandygriff, E.: Processing and characterization of NiTi porous SMA by elevated pressure sintering. Journal of Intelligent Material Systems and Structures 13, 837-850 (2002)

235. Lagoudas, D.C., Bo, Z.: Stress induced phase transformations in piezoelectric laminates with shape memory alloy layers. Transactions of the AMSE, Applied Mechanics Division 167, 107-118 (1993)

236. Lagoudas, D.C., Bo, Z.: The cylindrical bending of composite plates with piezoelectric and SMA layers. Smart Materials and Structures 3, 309-317 (1994)

237. Lagoudas, D.C., Boyd, J.G., Bo, Z.: Micromechanics of active composites with SMA fibers. Journal of Engineering Materials and Technology 116, 337-347 (1994)

238. Lagoudas, D.C., Entchev, P.B., Popov, P., Patoor, E., Brinson, L.C., Gao, X.: Shape memory alloys, Part II: Modeling of polycrystals. Mechanics of Materials 38(5-6), 430-462 (2006)

239. Lagoudas, D.C., Gavazzi, A.C., Nigam, H.: Elastoplastic behavior of metal matrix composites based on incremental plasticity and the Mori-Tanaka averaging scheme. Computational Mechanics 8, 193-203 (1991)

240. Lagoudas, D.C., Moorthy, D., Qidwai, M.A., Reddy, J.N.: Modeling of the thermomechanical response of active laminates with SMA strips using the layerwise finite element method. Journal of Intelligent Material Systems and Structures 8, 476-488 (1997)

241. Lagoudas, D.C., Tadjbakhsh, I.G.: Active flexible rods with embedded SMA fibers. Smart Materials and Structures 1, 162-167 (1992)

242. Lagoudas, D.C., Tadjbakhsh, I.G.: Deformations of active flexible rods with embedded line actuators. Smart Materials and Structures 2, 71-81 (1993)

243. Lahelin, M., Aaltio, I., Heczko, O., Söderberg, O., Ge, Y., Löfgren, B., Hannula, S.P., Seppälä, J.: DMA testing of Ni-Mn-Ga/polymer composites. Composites: Part A 40, 125-129 (2009)

244. Lebensohn, R.A., Ponte Casta neda, P., Brenner, R., Castelnau, O.: Full-field vs. homogenization methods to predict microstructure-property relations for polycrystalline materials. In: S. Ghosh, D. Dimiduk (eds.) Computational Methods for Microstructure-Property Relationships, pp. 393-441. Springer-Verlag, New York (2011)

245. Leclerc, H., Pri, J.N., Roux, S., Hild, F.: Voxel-scale digital volume correlation. Experimental Mechanics 51, 479-490 (2011) 
246. Lee, J., Hwang, J., Lee, D., Ryu, H.J., Hong, S.H.: Enhanced mechanical properties of spark plasma sintered NiTi composites reinforced with carbon nanotubes. Journal of Alloys and Compounds 617, 505-510 (2014)

247. Lee, J.K., Taya, M.: Strengthening mechanism of shape memory alloy reinforced metal matrix composite. Scripta Materialia 51, 443-447 (2004)

248. Lee, J.K., Taya, M.: Modeling for piezoelectric-shape memory alloy composites. Archive of Applied Mechanics 81, 629-640 (2011)

249. Lei, H., Wang, Z., Zhou, B., Tong, L., Wang, X.: Simulation and analysis of shape memory alloy fiber reinforced composite based on cohesive zone model. Materials and Design 40, 138-147 (2012)

250. Lelieveld, C.M.J.L., Jansen, K.M.B.: Thermal-electric characterization and modelling of a smart composite structure for architectural applications. Smart Materials and Structures 23 (2014). Art. no. 065010

251. Leng, J., Lan, X., Liu, Y., Du, S.: Shape-memory polymers and their composites: Stimulus methods and applications. Progress in Materials Science 56, 1077-1135 (2011)

252. Lester, B.T., Chemisky, Y., Lagoudas, D.C.: Transformation characteristics of SMA composites. Smart Materials and Structures 20, 094,002 (2011)

253. Lester, B.T., Kothalkar, A., Karaman, I., Radovic, M., Lagoudas, D.C.:

254. Levitas, V.I., Ozsoy, I.B.: Thermomechanical modeling of stress-induced transformations. Part 1. thermodynamics and kinetics of coupled interface propagation and reorientation. International Journal of Plasticity 25, 239-280 (2009)

255. Lewis, A.C., Geltmacher, A.B.: Image-based modeling of the response of experimental 3D microstructures to mechanical loading. Scripta Materialia 55, 81-85 (2006)

256. Lewis, A.C., Qidwai, S.M., Jackson, M., Geltmacher, A.B.: Strategies for of 3-D experimental data with modeling and simulation. Journal of the Minerals, Metals, and Materials Society 63(3), 35-39 (2011)

257. Li, B.Y., Rong, L.J., Li, Y.Y.: Porous NiTi prepared from elemental powder sintering. Journal of Materials Research 13(10), 2847-2851 (1998)

258. Li, B.Y., Rong, L.J., Li, Y.Y.: Fabrication of cellular NiTi inter metallic compounds. Journal of Materials Research 15(1), 10-13 (2000)

259. Li, B.Y., Rong, L.J., Li, Y.Y., Gjunter, V.: A recent development in producing porous Ni-Ti shape memory alloys. Intermetallics 8, 881-884 (2000)

260. Li, D.S., Zhang, X.P., Mai, Y.W.: Lightweight NiTi shape memory alloy based composites with high damping capacity and high strength. Journal of Alloys and Compounds 490, L15-L19 (2010)

261. Li, D.S., Zhang, Y.P., Eggeler, G., Zhang, X.P.: High porosity and high-strength porous NiTi shape memory alloys with controllable pore characteristics. Journal of Alloys and Compounds 470, L1-L5 (2009)

262. Li, J., Wang, H., Liu, J., Ruan, J.: Effects of $\mathrm{Nb}$ addition on microstructure and mechanical properties of TiNiNb alloys fabricated by elemental powder sintering. Materials Science \& Engineering A 609, 235-240 (2014)

263. Li, J.F., Zhang, Z.Q., Li, X.W., Peng, Z.W.: Application of shape memory alloy TiNi in low thermal expansion copper composites. Materials and Design 30, 314-318 (2009)

264. Li, X., Li, M., Song, G.: Energy-dissipating and self-repairing SMA-ECC composite material system. Smart Materials and Structures 24, 025,024 (2015)

265. Li, Y.H., Rong, L.J., Li, Y.Y.: Pore characteristics of porous NiTi alloy fabricated by combustion synthesis. Journal of Alloys and Compounds 325, 259-262 (2001)

266. Li, Y.H., Rong, L.J., Li, Y.Y.: Compressive property of porous NiTi alloy synthesized by combustion synthesis. Journal of Alloys and Compounds 345, 271-274 (2002)

267. Liang, C., Jia, J., Rogers, C.A.: Behavior of shape memory alloy reinforced composite plates Part II: Results. In: Proceedings of the $30^{\text {th }}$ AIAA/ASME/ASCE/AHS/ACS Structures, Structural Dynamics and Materials Conference, vol. 1331, pp. 1504-1513 (1989)

268. Liang, C., Rogers, C.A., Fuller, C.R.: Acoustic transmission and radiation analysis of adaptive shapememory alloy reinforced laminated plates. Journal of Sound and Vibration 145(1), 23-41 (1991)

269. Liang, Y., Kuga, Y., Taya, M.: Design of membrane actuator based on ferromagnetic shape memory alloy composite for synthetic jet applications. Sensors and Actuators A 125, 512-518 (2006)

270. Liang, Y., Taya, M., Xiao, J.Q., Xiao, G.: Design of an inchworm actuator based on a ferromagnetic shape memory alloy composite. Smart Materials and Structures 21, 115,005 (2012)

271. Liu, D.M., Nie, Z.H., Wang, G., Wang, Y.D., Brown, D.E., Pearson, J., Liaw, P.K., Ren, Y.: In-situ studies of stress- and magnetic-field-induced phase transformation in a polymer-bonded Ni-Co-Mn-In composite. Materials Science \& Engineering A 557, 3561-3571 (2010)

272. Liu, J., Scheerbaum, N., Kauffmann-Weiss, S., Gutfleisch, O.: NiMn-based alloys and composites for magnetically controlled dampers and actuators. Advanced Engineering Materials 14(8), 653-667 (2012). DOI 10.1002/adem.201200038

273. Liu, J., Scheerbaum, N., Weiß, S., Gutfleisch, O.: Ni-Mn-In-Co single-crystalline particles for magnetic shape memory composites. Applied Physics Letters 95, 152,503 (2009). DOI 10.1063/1.3249585

274. Liu, L., Lin, C., Zhou, C.: The constrained stress-induced $\varepsilon$ reverse martensitic transformation characteristic of Fe-Mn-Si alloy embedded in cement matrix. Applied Mechanics and Materials 44-47, 2229-2233 (2011)

275. Liu, X., Wu, S., Yeung, K.W.K., Chan, Y.L., Hu, T., Xu, Z., Liu, X., Chung, J.C.Y., Cheung, K.M.C., Chu, P.K.: Relationship between osseointegration and superplastic biomechanics in porous NiTi scaffolds. Biomaterials 32, 330-338 (2011) 
276. Liu, Y., Al-Matar, B., Newaz, G.: An investigation on the interface in a NiTi short-fiber-reinforced 6061 aluminum composite by transmission electron microscope. Metallurgical and Material Transactions A 39A, 2749-2759 (2008)

277. Liu, Z., Cui, L., Liu, Y., Jiang, D., Jiang, J., Shi, X., Shao, Y., Zheng, Y.: Influence of internal stress coupling on the deformation behavior of NiTi-Nb nanowire composites. Scripta Materialia 77, 75-78 (2014)

278. Liu, Z., Cui, L., Yu, C., Jiang, J., Jiang, D., Shi, X., Guo, F., Wang, Z., Ren, Y.: Influence of annealing and pre-straining on the coupling effect of a TiNi-Nb nanowire composite. Materials Science Forum 787, 307-312 (2014)

279. Liu, Z., Liu, Y., Jiang, D., Yang, F., Hao, S., Ren, Y., Cui, L.: Local strain matching between Nb nanowire and a phase transforming NiTi matrix in an in-situ composite. Materials Science \& Engineering A 610, 6-9 (2014)

280. López, G.A., Barrado, M., Bocanegra, E.H., San Juan, J.M., Nó, M.L.: Influence of the matrix and of the thermal treatment on the martensitic transformation in metal matrix composites. Materials Science \& Engineering A 481-482, 546-550 (2008)

281. López, G.A., Barrado, M., San Juan, J., Nó, M.L.: Mechanical spectroscopy measurements on SMA high-damping composites. Materials Science \& Engineering A 521-522, 359-362 (2009)

282. López, G.A., Barrado, M., San Juan, J.M., Nó, M.L.: Interaction of Cu-Al-Ni shape memory alloys particles with molten In and In+Sn matrices. Materials Science \& Engineering A 495, 304-309 (2008)

283. Lu, P., Cui, F.S., Tan, M.J.: A theoretical model for the bending of a laminated beam with SMA fiber embedded layer. Composite Structures 90, 458-464 (2009)

284. Lu, Z., Weng, G.: A two-level micromechanical theory for a shape-memory alloy reinforced composite. International Journal of Plasticity 16(10-11), 1289-1307 (2000)

285. Ma, J., Karaman, I., Noebe, R.D.: Medical applications of shape memory alloys. International Materials Reviews 55(5), 257-315 (2010)

286. Machado, L., Savi, M.: Medical applications of shape memory alloys. Brazilian Journal of Medical and Biological Research 36, 683-691 (2003)

287. Mahendran, M., Feuchtwanger, J., Techapiesancharoenkij, R., Bono, D., O'Handley, R.C.: Acoustic energy absorption in Ni-Mn-Ga/polymer composites. Journal of Magnetism and Magnetic Materials 323, 1098$1100(2011)$

288. Majumder, P., Bhattacharyya, A.: On the anisotropic thermal conductivity of shape memory alloy single crystals. Acta Mechanica 193, 5347-5365 (2007)

289. Manzo, J., Garcia, E.: Methodology for design of an active rigidity joint. Journal of Intelligent Material Systems and Structures 20, 311-327 (2009)

290. Marfia, S.: Micro-macro analysis of shape memory alloy composites. International Journal of Solids and Structures 42, 3677-3699 (2005)

291. Marfia, S., Sacco, E.: Analysis of SMA composite laminates using a multi scale modelling technique. International Journal for Numerical Methods in Engineering 70, 1182-1208 (2007)

292. Marfia, S., Sacco, E.: Micromechanics and homogenization of SMA-wire reinforced materials. Journal of Applied Mechanics 72, 259-268 (2007). DOI 10.1115/1.1839186

293. Mari, D., Dunand, D.C.: NiTi and NiTi-TiC composites: Part I. transformation and thermal cycling behavior. Metallurgical and Material Transactions A 26A, 2833-2847 (1995)

294. McDowell, D.L., Ghosh, S., Kalidindi, S.R.: Representation and computational structure-property relations of random media. Journal of the Minerals, Metals, and Materials Society 63(3), 45-51 (2011)

295. Meo, M., Antonucci, E., Duclaux, P., Giordano, M.: Finite element simulation of low velocity impact on shape memory alloy composite plates. Composite Structures 71, 337-342 (2005)

296. Meo, M., Marulo, F., Guida, M., Russo, S.: Shape memory alloy hybrid composites for improved impact properties for aeronautical applications. Composite Structures 95, 756-766 (2013)

297. Meraghni, F., Chemisky, Y., Piotrowski, B., Echchorfi, R., Bourgeois, N., Patoor, E.: Parameter identification of a thermodynamic model for superelastic shape memory alloys using analytical calculation of the sensitivity matrix. European Journal of Mechanics - A/Solids 45, 226-237 (2014)

298. Mete Ozturk, M., Bhattacharyya, A.: Heat transfer in shape memory alloy thin films. Acta Mechanica 225, 1029-1042 (2014). DOI 10.1007/s00707-013-1074-0

299. Miranda, G., Carvalho, O., Silva, F.S., Soares, D.: Effect of sintering stage in NiTi short-fibre-reinforced aluminum-silicon composites interface properties. Journal of Composite Materials 47(13), 1625-1631 (2013). DOI 10.1177/0021998312339893

300. Mizuuchi, K., Inoue, K., Hamada, K., Sugioka, M., Itami, M., Fukusumi, M., Kawahara, M.: Processing of TiNi SMA fiber reinforced AZ31 Mg alloy matrix composite by pulsed current hot pressing. Materials Science \& Engineering A 367, 343-349 (2004)

301. Monroe, J.A., Cruz-Perez, J., Yegin, C., Karaman, I., Geltmacher, A.B., Everett, R.K., Kainuma, R.: Magnetic response of porous NiCoMnSn metamagnetic shape memory alloys fabricated using solid-state replication. Scripta Materialia 67, 116-119 (2012)

302. Morgan, N.B.: Medical shape memory alloy applications - the market and its products. Materials Science \& Engineering A 378, 16-23 (2004)

303. Mori, T., Tanaka, K.: Average stress in matrix and average elastic energy of materials with misfitting inclusions. Acta Metallurgica 21, 571-574 (1973)

304. Morin, C., Moumni, Z., Zaki, W.: Thermomechanical coupling in shape memory alloys under cyclic loadings: Experimental analysis and constitutive modeling. International Journal of Plasticity 27, 1959 $1980(2011)$ 
305. Mura, T.: Micromechanics of Defects in Solids, 2nd revised edn. Mechanics of Elastic and Inelastic Solids. Kluwer Academic Publisher, Dordrecht (1987)

306. Murasawa, G., Tohgo, K., Ishii, H.: The effect of fiber volume fraction and aspect ratio on the creation of internal stress in the matrix and deformation for short-fiber shape memory alloy composites. Smart Materials and Structures 15, 33-40 (2006)

307. Murasawa, G., Yoneyama, S.: Local strain distribution arising in shape memory alloy composite subjected to thermal loading. Materials Transactions, JIM 47(3), 766-771 (2006)

308. Naghashian, S., Fox, B.L., Barnett, M.R.: Actuation curvature limited for a composite beam with embedded shape memory alloy wires. Smart Materials and Structures 23, 065,002 (2014)

309. İpek Nakaş, G., Dericioglu, A.F., Bor, c.: Fatigue behavior of TiNi foams processed by the magnesium space holder technique. Journal of the Mechanical Behavior of Biomedical Materials 4, 2017-2023 (2011)

310. İpek Nakaş, G., Dericioğlu, A.F., Bor, c.: Monotonic and cyclic compressive behavior of superelasitc TiNi foams processed by sintering using magnesium space holder technique. Materials Science \& Engineering A 582, 140-146 (2013)

311. Namli, O., Taya, M.: Design of piezo-SMA composite for thermal energy harvester under fluctuating temperature. Journal of Applied Mechanics 78, 031,001 (2011)

312. Nemat-Nasser, S., Sui, Y., Guo, W., Isaacs, J.: Experimental characterization and micromechanical modeling of superelastic response of a porous NiTi shape-memory alloy. Journal of the Mechanics and Physics of Solids 53, 2320-2346 (2005)

313. Neuking, K., Abu-Zarifa, A., Eggeler, G.: Surface engineering of shape memory alloy/polymer-composites: Improvement of the adhesion between polymers and pseudoelastic shape memory alloys. Materials Science \& Engineering A 7(11), 606-611 (2005)

314. Neuking, K., Abu-Zarifa, A., Eggeler, G.: Polymer/NiTi-composites: Fundamental aspects, processing and properties. Advanced Engineering Materials 481-482, 1014-1023 (2008)

315. Neurohr, A.J., Dunand, D.C.: Mechanical anisotropy of shape-memory NiTi with two-dimensional networks of micro-channels. Acta Materialia 59, 4616-4630 (2011)

316. Neurohr, A.J., Dunand, D.C.: Shape-memory NiTi with two-dimensional networks of micro-channels. Acta Biomaterialia 7, 1862-1872 (2011)

317. Neuser, S., Michaud, V.: Fatigue response of solvent-based self-healing smart materials. Experimental Mechanics 54, 293-304 (2014)

318. Neuser, S., Michaud, V., White, S.R.: Improving solvent-based self-healing materials through shape memory alloys. Polymer 53, 370-378 (2012)

319. Ni, D.R., Wang, J.J., Zhou, Z.N., Ma, Z.Y.: Fabrication and mechanical properties of bulk NiTip/Al composites prepared by friction stir processing. Journal of Alloys and Compounds 586, 368-374 (2014)

320. Ni, Q.Q., Zhang, R.X., Natsuki, T., Iwamoto, M.: Stiffness and vibration characteristics of SMA/ER3 composites with shape memory alloy short fibers. Composite Structures 79, 501-507 (2007)

321. Oehler, S.D., Hartl, D.J., Lopez, R., Malak, R.J., Lagoudas, D.C.: Design optimization and uncertainty analysis of SMA morphing structures. Smart Materials and Structures 21, 094,016 (2012)

322. Okazaki, T., Mikami, K., Furuya, Y., Kishi, Y., Yajima, Z., Kubota, T.: Magnetic properties of thin-film $\mathrm{Fe}-\mathrm{Pd}$ alloy and magnetoelectric coupling in $\mathrm{Fe}-\mathrm{Pd} / \mathrm{Ag} / \mathrm{PZT} / \mathrm{Ag} / \mathrm{Fe}-\mathrm{Pd}$ laminate composites. Journal of Alloys and Compounds 577, S300-S304 (2013)

323. Oppenheimer, S.M., Dunand, D.C.: Porous NiTi by creep expansion of argon-filled pores. Materials Science \& Engineering A 523, 70-76 (2009)

324. Otsuka, K., Ren, X.: Physical metallurgy of Ti-Ni-based shape memory alloys. Progress in Materials Science 50, 511-678 (2005)

325. Otsuka, K., Wayman, C.M. (eds.): Shape Memory Materials. Cambridge University Press, Cambridge (1999)

326. Ozbulut, O.E., Hurlebaus, S., Desroches, R.: Seismic response control using shape memory alloys: A review. Journal of Intelligent Material Systems and Structures 22, 1531 (2011)

327. Paine, J.S.N., Jones, W.M., Rogers, C.A.: NiTiNOL actuator to host composite interfacial adhesion in adaptive hybrid composites. AIAA Journal 2405, 556-565 (1992)

328. Paine, J.S.N., Rogers, C.A.: The effect of thermoplastic composite processing on the performance of embedded nitinol actuators. Journal of Thermoplastic Composite Materials 4, 102-122 (1991)

329. Paine, J.S.N., Rogers, C.A.: High velocity impact response of composites with surface bonded nitinol-SMA hybrid layers. Journal of Intelligent Material Systems and Structures 5(4), 530-535 (1994)

330. Paine, J.S.N., Rogers, C.A.: Review of multi-functional SMA hybrid composite materials and their applications. In: Proceedings of the American Society of Mechanical Engineers, Aerospace Division - Adaptive Structures and Composite Materials: Analysis and Application, vol. 54. ASME (1994)

331. Paine, J.S.N., Rogers, C.A.: Shape memory alloys for damage resistant composite structures. In: Proceedings of the SPIE: Active Materials and Smart Structures, vol. 2427, pp. 358-271. SPIE (1994)

332. Paine, J.S.N., Rogers, C.A., Smith, R.A.: Adaptive composite materials with shape memory alloy actuators for cylinders and pressure vessels. Journal of Intelligent Material Systems and Structures 6(4), 210-219 (1995)

333. Paiva, A., Savi, M.A., Bragra, A.M.B., Pacheco, P.M.C.L.: A constitutive model for shape memory alloys considering tensile-compressive asymmetry and plasticity. International Journal of Solids and Structures 42(11-12), 3439-3457 (2005)

334. Panico, M., Brinson, L.: A three-dimensional phenomenological model for martensite reorientation in shape memory alloys. Journal of the Mechanics and Physics of Solids 55, 2491-2511 (2007)

335. Panico, M., Brinson, L.C.: Computational modeling of porous shape memory alloys. International Journal of Solids and Structures 45, 5613-5626 (2008) 
336. Pappadà, S., Gren, P., Tatar, K., Gustafson, T., Rametta, R., Rossini, E., Maffezzoli, A.: Mechanical and vibration characteristics of laminated composite plates embedding shape memory alloy superplastic wires. Journal of Materials Engineering and Performance 18(5-6), 531-664 (2009)

337. Pappadà, S., Rametta, R., Largo, A., Maffezzoli, A.: Low-velocity impact response in composite plates embedding shape memory alloy wires. Polymer Composites 33(5), 655-664 (2012)

338. Pappadà, S., Rametta, R., Toia, L., Coda, A., Fumagalli, L., Maffezzoli, A.: Embedding of superplastic SMA wires into composite structures: Evaluation of impact properties. Journal of Materials Engineering and Performance 18(5-6), 522-630 (2009). DOI 10.1007/s11665-009-9366-1

339. Park, J.S., Kim, S.H., Jung, S.N.: Optimal design of a variable-twist prop rotor incorporating shape memory alloy hybrid composites. Composite Structures 93, 2288-2298 (2011)

340. Park, J.S., Kim, S.H., Jung, S.N., Lee, M.K.: Design and analysis of variable-twist tilt rotor blades using shape memory alloy hybrid composites. Smart Materials and Structures 20, 015,001 (2011)

341. Park, Y.C., Jo, Y.J., Baek, S.H., Furuya, Y.: Fatigue design curve of a TiNi/Al shape memory alloy composite for aircraft stringer design. Smart Materials and Structures 18, 055,009 (2009)

342. Park, Y.C., Kang, J.H., Lee, J.K., Lee, G.C., Furuya, Y.: Effect of cold rolling on fatigue crack propagation of TiNi/Al6061 shape memory composite. Smart Materials and Structures 16, 982-988 (2007)

343. Park, Y.C., Lee, J.K., Lee, S.P., Lee, G.C., Lee, J.H., Cho, Y.H., Lee, J.B.: A study on fatigue damage of shape memory alloy composite using NDE technique. International Journal of Modern Physics B 20(25-27), 3775-3780 (2006)

344. Patoor, E., Eberhardt, A., Berveiller, M.: Micromechanical modelling of superelasticity in shape memory alloys. Journal de Physique IV 6, C1-277-292 (1996)

345. Patoor, E., Lagoudas, D.C., Entchev, P.B., Brinson, L.C., Gao, X.: Shape memory alloys, Part I: General properties and modeling of single crystals. Mechanics of Materials 38(5-6), 391-429 (2006)

346. Payandeh, Y., Meraghni, F., Patoor, E., Eberhardt, A.: Effect of martensitic transformation on the debunking propagation in Ni-Ti shape memory wire composite. Materials Science \& Engineering A 518, 35-40 (2009)

347. Payandeh, Y., Meraghni, F., Patoor, E., Eberhardt, A.: Debonding initiation in a NiTi shape memory wire-epoxy matrix composite. influence of martensitic transformation. Materials and Design 31, 1077$1084(2010)$

348. Payandeh, Y., Meraghni, F., Patoor, E., Eberhardt, A.: Study of the martensitic transformation in NiTiepoxy smart composite and its effect on the overall behavior. Materials and Design 39, 104-110 (2012)

349. Peraza Hernandez, E., Hartl, D.J., Galvan, E., Malak, R.: Design and optimization of a shape memory alloy-based self-folding sheet. Journal of Mechanical Design 135(11), 111,007 (2013). DOI $10.1115 / 1.4025382$

350. Peraza Hernandez, E.A., Hartl, D.J., Malak, R.J.J.: Design and numerical analysis of an SMA meshbased self-folding sheet. Smart Materials and Structures 22, 094,008 (2013). DOI 10.1088/0964$1762 / 22 / 9 / 094008$

351. Peraza Hernandez, E.A., Hu, S., Kung, H.W., Hartl, D., Akleman, E.: Towards building smart self-folding structures. Computers \& Graphics 37(6), 730-742 (2013)

352. Pierard, O., González, C., Seguarado, J., LLorca, J., Doghri, I.: Micromechanics of elasto-plastic materials reinforced with ellipsoidal inclusions. International Journal of Solids and Structures 44, 6945-6962 (2007)

353. Pierard, O., LLorca, J., Seguarado, J., Doghri, I.: Micromechanics of particle-reinforced elastovicscoplastic composites: Finite element simulations versus affine homogenization. International Journal of Plasticity 23, 1041-1060 (2007)

354. Pindera, M.J., Khatam, H., Drago, A., Bansal, Y.: Micromechanics of spatially uniform heterogeneous media: A critical review and emerging approaches. Composites Part B: Engineering 40(5), 349-378 (2009)

355. Pinto, F., Ciampa, F., Meo, M., Polimeno, U.: Multifunctional SMArt composite material for in situ NDT/SHM and de-icing. Smart Materials and Structures 21, 105,010 (2012)

356. Pinto, F., Meo, M.: Mechanical response of shape memory alloy-based hybrid composite subjected to lowvelocity impacts. Journal of Composite Materials p. In Press (2014). DOI 10.1177/0021998314554119

357. Piotrowski, B., Ben Zineb, T., Patoor, E., Eberhardt, A.: A finite element-based numerical tool for $\mathrm{Ni}_{47} \mathrm{Ti}_{44} \mathrm{Nb}_{9}$ SMA structures design: application to tightening rings. Journal of Intelligent Material Systems and Structures 23, 141-153 (2012)

358. Piotrowski, B., Ben Zineb, T., Patoor, E., Eberhardt, A.: Modeling of niobium precipitates effect on the $\mathrm{Ni}_{47} \mathrm{Ti}_{44} \mathrm{Nb}_{9}$ shape memory alloy behavior. International Journal of Plasticity 36, 130-147 (2012)

359. Pons, J., Cesari, E., Seguí, C., Masdeu, F., Santamarta, R.: Ferromagnetic shape memory alloys: Alternatives to Ni-Mn-Ga. Materials Science \& Engineering A 481-482, 57-65 (2008)

360. Poon, C.k., Lau, K.t., Zhou, L.m.: Design of pull-out stresses for prestrained SMA wire/polymer hybrid composites. Composites: Part B 36, 25-31 (2005)

361. Porter, G.A., Liaw, P.K., Tiegs, T.N., Wu, K.H.: Ni-Ti SMA-reinforced Al composites. Journal of the Minerals, Metals, and Materials Society 52(10), 52-56 (2000)

362. Porter, G.A., Liaw, P.K., Tiegs, T.N., Wu, K.H.: Particle size reduction of NiTi shape-memory alloy powders. Scripta Materialia 43, 1111-1117 (2000)

363. Porter, G.A., Liaw, P.K., Tiegs, T.N., Wu, K.H.: Fatigue and fracture behavior of nickel-titanium shape memory alloy reinforced aluminum composites. Materials Science \& Engineering A 314(10), 186-193 (2001)

364. Prahlad, H., Chopra, I.: Development of a strain-rate dependent model for uniaxial loading of SMA wires. Journal of Intelligent Material Systems and Structures 14(14), 429-442 (2003)

365. Qidwai, M.A., DeGiorgi, V.G.: Numerical assessment of the dynamic behavior of hybrid shape memory alloy composite. Smart Materials and Structures 13, 134-145 (2004) 
366. Qidwai, M.A., Entchev, P.B., Lagoudas, D.C., DeGiorgi, V.G.: Modeling of the thermomechanical behavior of porous shape memory alloys. International Journal of Solids and Structures 38, 8653-8671 (2001)

367. Qidwai, M.A., Lagoudas, D.C.: On the thermodynamics and transformation surfaces of polycrystalline NiTi shape memory alloy material. International Journal of Plasticity 16, 1309-1343 (2000)

368. Qidwai, M.A.S., Lewis, A.C., Geltmacher, A.B.: Using image-based computational modeling to study microstructure-yield correlations in metals. Acta Materialia 57, 4233-4247 (2009)

369. Qidwai, S.M., Turner, D.M., Niezgoda, S.R., Lewis, A.C., Geltmacher, A.B., Rowenhorst, D.J., Kalidindi, S.R.: Estimating the response of polycrystalline materials using sets of weighted statistical volume elements. Acta Materialia 60, 5284-5299 (2012)

370. Qiu, Z.X., Yao, X.T., Yuan, J., Soutis, C.: Experimental research on strain monitoring in composite plates using embedded SMA wires. Smart Materials and Structures 48, 1047-1053 (2006)

371. Qu, J., Cherkaoui, M.: Fundamentals of Micromechanics of Solids. John Wiley \& Sons, Inc., Hoboken, NJ (2006)

372. Raghavan, J., Bartkiewicz, T., Boyko, S., Kupriyanov, M., Rajapakse, N., Yu, B.: Damping, tensile, and impact properties of super elastic shape memory alloy (SMA) fiber-reinforced polymer composites. Composites: Part B 41, 214-222 (2010)

373. Ratna, D., Karger-Kocsis, J.: Recent advances in shape memory polymers and composites: a review. Journal of Materials Science 43, 254-269 (2008)

374. Rey, T., Fazan, F., Robin, E., Faure, S., Le Cam, J.B., Chignon, G., Girard, A., Favier, D.: Mechanical characterization and comparison of different $\mathrm{NiTi} /$ silicone rubber interfaces. International Journal of Adhesion \& Adhesives 48, 67-74 (2014)

375. Ro, J., Baz, A.: NITINOL-reinforced plates: Part I. thermal characteristics. Composites Engineering 5(1), 61-75 (1995)

376. Ro, J., Baz, A.: NITINOL-reinforced plates: Part II. static and buckling characteristics. Composites Engineering 5(1), 77-90 (1995)

377. Ro, J., Baz, A.: NITINOL-reinforced plates: Part III. dynamic characteristics. Composites Engineering 5(1), 91-106 (1995)

378. Ro, J., Baz, A.: Control of sound radiation from a NiTiNOL-reinforced plate into an acoustic cavity. In: Proceedings of SPIE, Smart Structures and Materials, vol. 2715, pp. 220-232. San Diego, CA (1996)

379. Rogers, C.A., Barker, D.K.: Experimental studies of active strain energy tuning of adaptive composites. In: Proceedings of the $31^{\text {st }}$ AIAA/ASME/ASCE/AHS/ASC Structures, Structural Dynamics and Materials Conference, pp. 2234-2241 (1990)

380. Rogers, C.A., Fuller, C.R., Liang, C.: Active control of sound radiation from panels using embedded shape memory alloy fibers. Journal of Sound and Vibration 136(1), 164-170 (1990)

381. Rogers, C.A., Liang, C., Fuller, C.R.: Modeling of shape memory alloy hybrid composites for structural acoustic control. Journal of the Acoustical Society of America 89(1), 210-220 (1990)

382. Rogers, C.A., Liang, C., Jia, J.: Behavior of shape memory alloy reinforced composite plates Part I: Model formulations and control concepts. In: Proceedings of the $30^{t h}$ AIAA/ASME/ASCE/AHS/ASC Structures, Structural Dynamics and Materials Conference, pp. 2011-2017 (1989)

383. Rogers, C.A., Robertshaw, H.H.: Development of a novel smart material. In: Proceedings of the 1988 Winter Annual Meeting of the American Society of Mechanical Engineers, pp. 1-5 (1988)

384. Romberg, J., Hürrich, C., Pötschke, M., Kauffmann-Weiss, S., Gaitzsch, U., Roth, S., Müllner, P., Schultz, L.: Geometric factors on magnetically driven actuation behavior for polycrystalline Ni-Mn-Ga and its composites. Journal of Alloys and Compounds 577S, S344-S347 (2013)

385. Rossi, S., Deflorian, F., Pegoretti, A., D'Orazio, D., Gialanella, S.: Chemical and mechanical treatments to improve the surface properties of shape memory NiTi wires. Surface \& Coatings Technology 22, 2214-2222 (2008)

386. Ryan, G., Pandit, A., Apatsidis, D.: Fabrication methods of porous metals for use in orthopaedic applications. Biomaterials 27(13), 2651-2670 (2006)

387. Ryu, J., Jung, B.S., Kim, M.S., Kong, J., Cho, M., Ahn, S.H.: Numerical simulation of hybrid composite shape-memory alloy wire-embedded structures. Journal of Intelligent Material Systems and Structures 22(17), 1941-1948 (2011)

388. Sadrnezhaad, S.K., Nemati, N.H., Bagheri, R.: Improved adhesion of NiTi wire to silicone matrix for smart composite medical applications. Materials and Design 30, 3667-3672 (2009)

389. Saint-Sulpice, L., Arbab Chirani, S., Calloch, S.: A 3D super-elastic model for shape memory alloys taking into account progressive strain under cyclic loadings. Mechanics of Materials 41, 12-26 (2009)

390. Samadpour, M., Sadighi, M., Shakeri, M., Zamani, H.: Vibration analysis of thermally buckled sma hybrid composite sandwich plate. Composite Structures 119, 251-263 (2015)

391. San Juan, J., Nó, M.L.: Internal friction in a new kind of metal matrix composites. Materials Science \& Engineering A 442, 429-432 (2006)

392. San Martín, D., Risanti, D.D., Garcés, G., Rivera-Diaz-del Castillo, P.E.J., van der Zwaag, S.: On the production and properties of novel particulate $\mathrm{NiTi}_{p} / \mathrm{AA} 2124$ metal matrix composites. Materials Science \& Engineering A 526, 250-252 (2009)

393. Sasikumar, P., Suresh, R., Gupta, S.: Stochastic finite element analysis of layered composite beams with spatially varying non-gaussian inhomogeneities. Acta Mechanica 225, 1503-1522 (2014). DOI 10.1007/s00707-013-1009-9

394. Saunders, W.R., Robertshaw, H.H., Rogers, C.A.: Structural acoustic control of a shape memory alloy composite beam. Journal of Intelligent Material Systems and Structures 2, 508-527 (1991) 
395. Savi, M.A., Paiva, A.: Describing internal subloops due to incomplete phase trasnformations in shape memory alloys. Archive of Applied Mechanics 74(9), 637-647 (2005)

396. Sawaguchi, T., Kikuchi, T., Ogawa, K., Kajiwara, S., Ikeo, Y., Kojima, M., Ogawa, T.: Development of prestressed concrete using Fe-Mn-Si-based shape memory alloys containing NbC. Materials Transactions, JIM 47(3), 580-583 (2006)

397. Scheerbaum, N., Hinz, D., Gutfleisch, O., Mülller, K.H., Schultz, L.: Textured polymer bonded composites with Ni-Mn-Ga magnetic shape memory particles. Acta Materialia 55, 2707-2713 (2007)

398. Segurado, J., LLorca, J.: Computational micromechanics of composites: The effect of particle spatial distribution. Mechanics of Materials 38, 873-883 (2006)

399. Seiani, H.A., Ghazavi, A.: A thermo-micro-mechanical modeling for smart shape memory alloy woven composite under in-plane biaxial deformation. International Journal of Mechanics and Materials in Design 5, 111-122 (2009)

400. Shaw, J., Kyriakides, S.: Thermomechanical aspects of NiTi. Journal of the Mechanics and Physics of Solids 43(8), 1243-1281 (1995)

401. Simpson, J.C., Boller, C.: Design and performance of a shape memory alloy-reinforced composite aerodynamic profile. Smart Materials and Structures 17 (2008). Art. no. 025028

402. Smith, C., Villanueva, A., Joshi, K., Tadesse, Y., Priya, S.: Working principle of bio-inspired shape memory alloy composite actuators. Smart Materials and Structures 20 (2011). Art. no. 012001

403. Smith, N.A., Anton, G.G., Ellis, A.B., Crone, W.C.: Improved adhesion between nickel-titanium shape memory alloy and a polymer matrix via silane coupling agents. Composites: Part A 35, 1307-1312 (2004)

404. Sofocleous, K., Ogin, S.L., Tsakiropoulos, P., Draconakis, V., Doumanidis, C.: Controlled impact testing of woven fabric composites with and without reinforcing shape-memory alloy wires. Journal of Composite Materials 48, 3799-3813 (2014)

405. Soghrati, S., Thakre, P.R., White, S.R., Sottos, N.R., Geubelle, P.H.: Computational modeling and design of actively-cooled microvascular materials. International Journal of Heat and Mass Transfer 55(19-20), 5309-5321 (2012)

406. Song, G., Ma, N., Li, H.N.: Applications of shape memory alloys in civil structures. Engineering Structures 28, 1266-1274 (2006)

407. Song, G., Sun, Q., Cherkaoui, M.: Role of microstructure in the thermomechanical behavior of sma composites. Journal of Engineering Materials and Technology 121(1), 86-92 (1999)

408. Strutt, E.R., Olevsky, E.A., Meyers, M.A.: Combustion synthesis/quasi-isostatic pressing of TiC-NiTi cermets: processing and mechanical response. Journal of Materials Science 43, 6513-6526 (2008)

409. Strutt, E.R., Radetic, T., Olevsky, E.A., Meyers, M.A.: Combustion synthesis/quasi-isostatic pressing of $\mathrm{TiC}_{0.7}$-NiTi cermets: microstructure and transformation characteristics. Journal of Materials Science $\mathbf{4 3}$, $5905-5923(2008)$

410. Sun, L., Huang, W.M., Ding, Z., Zhao, Y., Wang, C.C., Purnawali, H., Tang, C.: Stimulus-responsive shape memory materials: A review. Materials and Design 33, 577-640 (2012)

411. Taheri-Behrooz, F., Taheri, F., Hosseinzadeh, R.: Characterization of a shape memory alloy hybrid composite subject to static loading. Materials and Design 32, 2923-2933 (2011)

412. Tanaka, K., Nishimura, F., Hayashi, T., Tobushi, H., Lexcellent, C.: Phenomenological analysis on subloops and cyclic behavior in shape memory alloys under mechanical and/or thermal loads. Mechanics of Materials 19, 281-292 (1995)

413. Tang, W., Sundman, B., Sandström, R., Qiu, C.: New modelling of the B2 phase and its associated martensitic transformation in the Ti-Ni system. Acta Metallurgica 47(12), 3457-3468 (1999)

414. Taya, M., Liang, Y., Namli, O.C., Tamagawa, H., Howie, T.: Design of two-way reversible bending actuator based on a shape memory alloy/shape memory polymer composite. Smart Materials and Structures 22 (2013). Art. no. 105003

415. Thorat, R.R., Risanti, D.D., San Martín, D., Garces, G., Rivera Díaz del Catillo, P.E.J., van der Zwaag, S.: On the transformation behavior of NiTi particulate reinforced AA2124 composites. Journal of Alloys and Compounds 477, 307-315 (2009)

416. Tian, B., Chen, F., Tong, Y., Li, L., Zheng, Y.F.: Magnetic field induced strain and damping behavior of Ni-Mn-Ga particles/epoxy resin composite. Journal of Alloys and Compounds 604, 137-141 (2014)

417. Tian, B., Chen, F., Tong, Y.X., Li, L., Zheng, Y.F.: Bending properties of epoxy resin matrix composites filled with Ni-Mn-Ga ferromagnetic shape memory alloy powders. Materials Letters 63, 1729-1732 (2009)

418. Tian, B., Chen, F., Tong, Y.X., Li, L., Zheng, Y.F., Liu, Y.: The orientation dependence of transformation strain of Ni-Mn-Ga polycrystalline alloy and its composite with epoxy resin. Journal of Alloys and Compounds 505, 680-684 (2010)

419. Tobushi, H., Hayashi, S., Hoshio, K., Makino, Y., Miwa, N.: Bending actuation characteristics of shape memory composite with SMA and SMP. Journal of Intelligent Material Systems and Structures 17, 1075-1081 (2006)

420. Tobushi, H., Hayashi, S., Pieczyska, E., Date, K., Nishimura, Y.: Three-way actuation of shape memory composite. Archives of Mechanics 5-6, 443-457 (2011)

421. Tobushi, H., Hayashi, S., Sugimoto, Y., Date, K.: Performance of shape memory composite with SMA and SMP. Solid State Phenomena 154, 65-70 (2009)

422. Tobushi, H., Hayashi, S., Sugimoto, Y., Date, K.: Fabrication and two-way deformation of shape memory composite with SMA and SMP. Materials Science Forum 638-642, 2189-2194 (2010)

423. Tobushi, H., Pieczyska, E., Ejiri, Y., Sakuragi, T.: Thermomechanical properties of shape-memory alloy and polymer and their composites. Mechanics of Advanced Materials and Structures 16, 236-247 (2009) 
424. Torquato, S.: Random Heterogeneous Materials: Microstructure and Macroscopic Properties. Interdisciplinary Applied Mathematics: Mechanics and Materials. Springer Science \& Business Media, New York, NJ (2002)

425. Triantafyllou, G., Psarras, G.C.: Probing the reverse martensitic transformation in constrained shape memory alloys via electrical resistance. Journal of Intelligent Material Systems and Structures 21, 975$981(2010)$

426. Turner, T.L., Buehrle, R., Cano, R., Fleming, G.: Modeling, fabrication, and testing of a SMA hybrid composite jet engine chevron concept. Journal of Intelligent Material Systems and Structures 17, 483-497 (2006)

427. Turner, T.L., Patel, H.D.: Analysis of SMA hybrid composite structures in MSC.Nastran and ABAQUS. Journal of Intelligent Material Systems and Structures 18, 435-447 (2007)

428. Umezaki, E.: Temperature distributions of SMA wires embedded in epoxy resin plates and heated by supplying electric current. Journal of Intelligent Material Systems and Structures 17, 1115-1120 (2006)

429. Vaidyanathan, R., Bourke, M.A.M., Dunand, D.C.: Phase fraction, texture and strain evolution in superplastic NiTi and NiTi-TiC composites investigated by neutron diffraction. Acta Metallurgica 47(12), 3353-3366 (1999)

430. Vaidyanathan, R., Bourke, M.A.M., Dunand, D.C.: Stress-induced martensitic transformations in NiTi and $\mathrm{NiTi}-\mathrm{TiC}$ composites investigated by neutron diffraction. Materials Science \& Engineering A 273275, 404-409 (1999)

431. Vaidyanathan, R., Bourke, M.A.M., Dunand, D.C.: An in situ neutron diffraction mechanical study of superplastic NiTi and NiTi-TiC composites. Journal De Physique IV 112, 823-826 (2003)

432. Vaidyanathan, R., Dunand, D.C., Ramamurtny, U.: Fatigue crack-growth in shape memory NiTi and NiTi-TiC composites. Materials Science \& Engineering A 289, 208-216 (2000)

433. Villanueva, A., Smith, C., Priya, S.: A biomimetic robotic jellyfish (Robojelly) actuated by shape memory alloy composite actuators. Bioinspiration \& Biomimetics 6 (2011). Art. no. 036004

434. Villanueva, A.A., Joshi, K.B., Blottman, J.B., Priya, S.: A bio-inspired shape memory alloy composite (BISMAC) actuator. Smart Materials and Structures 19, 025,013 (2010)

435. Wang, Q., Cui, C., Yan, N.: Fabrication of a CuAlMn shape memory alloy by the sintering-dissolution process. Materials Letters 65, 2735-2738 (2011)

436. Wang, Q., Han, F., Wu, J., Hao, G.: Damping behavior of porous CuAlMn shape memory alloy. Materials Letters 61, 2598-2600 (2007)

437. Wang, Q., Lu, D., Cui, C., Yan, N., Wang, Q.: Fabrication and internal friction behaviors of novel porous CuAlMn shape memory alloy filled with polystyrene. Materials Letters 92, 82-85 (2013)

438. Wang, Q.Z., Han, F.S., Cui, C.: Effects of macroscopic graphite particulates on the damping behavior of CuAlMn. Journal of Materials Science 42, 5029-5035 (2007)

439. Wang, Q.Z., Han, F.S., Wu, J., Hao, G.L.: Thermal stresses in a macroscopic graphite particulates reinforced CuAlMn shape memory alloy studied by internal friction. Materials Science \& Engineering A 408, 247-254 (2005)

440. Wang, Q.Z., Lu, D.M., Cui, C.X., Liu, W.J., Xu, M., Yang, J.: Effects of aging on the structure and damping behaviors of a novel porous CuAlMn shape memory alloy fabricated by sintering-dissolution method. Materials Science \& Engineering A 615, 278-282 (2014)

441. Wang, S., Guo, F.M., Jiang, D.Q., Liu, Y., Cui, L.S.: In situ W-NiTi shape memory alloy composite of high radiopacity. Scripta Materialia 81, 4-7 (2014)

442. Wang, W., Lee, J.Y., Rodrigue, H., Song, S.H., Chu, W.S., Ahn, S.H.: Locomotion of inchworm-inspired robot made of smart soft composite (ssc). Bioinspiration \& Biomimetics 9, 046,006 (2014)

443. Wang, X., Hu, G.: Stress transfer for a SMA fiber pulled out from an elastic matrix and related bridging effect. Composites: Part A 36, 1142-1151 (2005)

444. Wang, Y., Zhou, L., Wang, Z., Huang, H., Ye, L.: Analysis of internal stresses induced by strain recovery in a single SMA fiber-matrix composite. Composites: Part B 42, 1135-1143 (2011)

445. Wang, Y., Zhou, L., Wang, Z., Huang, H., Ye, L.: Stress distributions in single shape memory alloy fiber composites. Materials and Design 32, 3783-3789 (2011)

446. Wang, Z.X., Dutta, I., Majumdar, B.S.: Thermal cycle response of a lead-free solder reinforced with adaptive shape memory alloy. Materials Science \& Engineering A 421, 133-142 (2006)

447. Wang, Z.X., Dutta, I., Majumdar, B.S.: Thermomechanical response of a lead-free solder reinforced with a shape memory alloy. Scripta Materialia 54, 627-632 (2006)

448. Wei, Z.G., Sandström, R., Miyazaki, S.: Shape memory materials and hybrid composites for smart systems: Part II shape-memory hybrid composites. Journal of Materials Science 33, 3763-3783 (1998)

449. White, S.R., Berman, J.B.: Thermomechanical response of SMA composite beams with embedded nitinol wires in an epoxy matrix. Journal of Intelligent Material Systems and Structures 9, 391-400 (1998)

450. Whitten, W., Hartl, D.: Iterative calibration of a shape memory alloy constitutive model from $1 \mathrm{~d}$ and $2 \mathrm{~d}$ experimental data using optimization methods. In: SPIE Smart Structures and Materials+ Nondestructive Evaluation and Health Monitoring, pp. 905,804-905,804. International Society for Optics and Photonics (2014)

451. Wierschem, N., Andrawes, B.: Superelastic SMA-FRP composite reinforcement for concrete structures. Smart Materials and Structures 19 (2010). Art. no. 025011

452. Witherspoon, C., Zheng, P., Chmielus, M., Vogel, S.C., Dunand, D.C., Müllner, P.: Texture and training of magnetic shape memory foam. Acta Materialia 61, 2113-2120 (2013)

453. Wu, R., Han, M.W., Lee, G.Y., Ahn, S.H.: Woven type smart soft composite beam with in-plane shape retention. Smart Materials and Structures 22 (2013). Art. no. 125007 
454. Xie, C.L., Hailat, M., Wu, X., Newaz, G.: Development of short fiber-reinforced NiTi/Al6061 composite. Journal of Engineering Materials and Technology 129, 69-76 (2007)

455. Xiong, J.Y., Li, Y.C., Wang, X.J., Hodgson, P.D., Wen, C.E.: Titanium-nickel shape memory alloy foams for bone tissue engineering. Journal of the Mechanical Behavior of Biomedical Materials 1(3), 269-273 $(2008)$

456. Xiu, Z., Laeng, J., Sun, X., Li, Q., Hur, S.K., Liu, Y.: Phase formation of $\mathrm{Al}_{2} \mathrm{O}_{3} / \mathrm{Ti}(\mathrm{C}, \mathrm{N})-\mathrm{NiTi}$ composite. Journal of Alloys and Compounds 458, 398-404 (2008)

457. Xu, J.L., Bao, L.Z., Liu, A.H., Jin, X.J., Tong, Y.X., Luo, J.M., Zhong, Z.C., Zheng, Y.F.: Microstructure, mechanical properties and superplasticity of biomedical porous NiTi alloy prepared by microwave sintering. Materials Science and Engineering C 46, 387-393 (2015)

458. Xu, J.L., Jin, X.F., Luo, J.M., Zhong, Z.C.: Fabrication and properties of porous NiTi alloys by microwave sintering for biomedical applications. Materials Letters 124, 110-112 (2014)

459. Xuan, H.C., Wang, L.Y., Zheng, Y.X., Cao, Q.Q., Deng, Y., Wang, D.H., Du, Y.W.: Large converse magnetoelectric effect in ferromagnetic shape memory alloy $\mathrm{Ni}_{49} \mathrm{Fe}_{18} \mathrm{Ga}_{27} \mathrm{Co}_{6}$ and $\mathrm{Pb}\left(\mathrm{Zr}_{0.52} \mathrm{Ti}_{0.48}\right) \mathrm{O}_{3}$ laminates. Journal of Alloys and Compounds 519, 97-100 (2012)

460. Yamada, Y., Taya, M., Watanabe, R.: Strengthening of metal matrix composite by shape memory effect. Materials Transactions, JIM 34(3), 5083-5091 (1993)

461. Yan, B., Li, G.: Mg alloy matrix composite reinforced with TiNi continuous fiber prepared by ballmilling/hot-pressing. Composites: Part A 36, 1590-1594 (2005)

462. Yang, R., Cui, L., Zheng, Y.: Synthesis of TiC/NiTi composite powders in molten salt and their sintering. Journal of Materials Science 43, 98-101 (2008)

463. Yang, S., Dui, G., Ma, B.: Temperature variation of a NiTi wire considering the effects of test machine grips. Acta Mechanica p. In Press (2015). DOI 10.1007/s00707-015-1322-6

464. Yen, F.C., Hwang, K.S., Wu, S.K.: Fabrication of porous Ti-rich $\mathrm{Ti}_{51} \mathrm{Ni}_{49}$ by evaporating $\mathrm{NaCl}$ space holder. Metallurgical and Material Transactions A 45A, 2626-2635 (2014). DOI 10.1007/s11661-0142196-2

465. Yongsheng, R., Shulian, Y., Xiaohui, W.: Structural modeling of SMA fiber hybrid active thin-walled composite beams. Composite Structures 91, 120-130 (2009)

466. Yoon, H.J., Costantini, D.M., Limberger, H.G., Salathé, R.P., Kim, C.G., Michaud, V.: In situ strain and temperature monitoring of adaptive composite materials. Journal of Intelligent Material Systems and Structures 17, 1059-1067 (2006)

467. Young, M.L., DeFouw, J.D., Frenzel, J., Dunand, D.C.: Cast-replicated NiTiCu foams with superplastic properties. Metallurgical and Material Transactions A 43A, 2939-2944 (2012)

468. Zafar, A., Andrawes, B.: Fabrication and cyclic behavior of highly ductile superelastic shape memory composites. Journal of Materials in Civil Engineering 26(4), 622-632 (2012). DOI 10.1061/(ASCE)MT.19435533.0000797

469. Zafar, A., Andrawes, B.: Incremental dynamic analysis of concrete moment resisting frames reinforced with shape memory composite bars. Smart Materials and Structures 21 (2012). Art. no. 025013

470. Zafar, A., Andrawes, B.: Experimental flexural behavior of SMA-FRP reinforced concrete beam. Frontiers of Structural and Civil Engineering 7(4), 341-355 (2013). DOI 10.1007/s11709-013-0221-y

471. Zakharov, D., Lebedev, G., Irzhak, A., Afonina, V., Mashirov, A., Kalashnikov, V., Koledov, V., Shelyakov, A., Podgorny, D., Tabachkova, N., Shavrov, V.: Submicron-sized actuators based on enhanced shape memory composite materials fabricated by FIB-CVD. Smart Materials and Structures 21 (2012). Art. no. 052001

472. Zeng, M., Or, S.W., Chan, H.L.W.: Effect of phase transformation on the converse magnetoelectric properties of a heterostructure of $\mathrm{Ni}_{49.2} \mathrm{Mn}_{29.6} \mathrm{Ga}_{21.2}$ and $0.7 \mathrm{PbMg}_{1 / 3} \mathrm{Nb}_{2 / 3} \mathrm{O}_{3}-0.3 \mathrm{PbTiO}_{3}$ crystals. Applied Physics Letters 96, 182,503 (2010). DOI 10.1063/1.3427388

473. Zeng, M., Or, S.W., Chan, H.L.W.: Large magnetoelectric effect from mechanically mediated magnetic field-induced strain effect in Ni-Mn-Ga single crystal and piezoelectric effect in PVDF polymer. Journal of Alloys and Compounds 490, L5-L8 (2010)

474. Zhang, R.X., Ni, Q.Q., Mauda, A., Yamamura, T., Iwamoto, M.: Vibration characteristics of laminated composite plates with embedded shape memory alloys. Composite Structures 74, 389-398 (2006)

475. Zhang, R.X., Ni, Q.Q., Natsuki, T., Iwamoto, M.: Mechanical properties of composites filled with SMA particles and short fibers. Composite Structures 79, 90-96 (2007)

476. Zhang, X.X., Hou, H.W., Wie, W.T., Wei, L.G.: High damping capacity in porous NiTi alloy with bimodal pore architecture. Journal of Alloys and Compounds 550, 297-301 (2013)

477. Zhang, X.X., Witherspoon, C., Müllner, P., Dunand, D.C.: Effect of pore architecture on magnetic-fieldinduced strain in polycrystalline ni-mn-ga. Acta Materialia 59, 2229-2239 (2011)

478. Zhang, Y., Li, D., Zhang, X.: Gradient porosity and large pore size niti shape memory alloys. Scripta Materialia 57, 1020-1023 (2007)

479. Zhang, Y., Zhao, Y.P.: A discussion on modeling shape memory alloy embedded in a composite laminate as axial force and elastic foundation. Materials and Design 28, 1016-1020 (2007)

480. Zhang, Y., Zhao, Y.P.: A study of composite beam with shape memory alloy arbitrarily embedded under thermal and mechanical loadings. Materials and Design 28, 1096-1115 (2007)

481. Zhao, L.M., Feng, X., Mi, X.J., Li, Y.F., Xie, H.F., Yin, X.Q.: The interfacial strength improvement of SMA composite using $\mathrm{ZnO}$ with electrochemical deposition method. Applied Surface Science 320, 670-673 (2014)

482. Zhao, X., Sun, H., Lan, L., Huang, J., Zhang, H., Wang, Y.: Pore structures of hit-porosity NiTI alloys made from elemental powders with NaCl temporary space-holders. Materials Letters 63, 2402-2404 (2009) 
483. Zhao, Y., M., T., Kang, Y., A., K.: Compression behavior of porous NiTi shape memory alloy. Acta Materialia 53, 337-343 (2005)

484. Zheng, P., Kucza, N.J., Wang, Z., Müllner, Dunand, D.C.: Effect of directional solidification on texture and magnetic-field-induced strain in Ni-Mn-Ga foams with coarse grains. Acta Materialia 86, 95-101 (2015)

485. Zheng, Y., Cui, L., Schrooten, J.: Thermal cycling behaviors of a NiTiCu wire reinforced Kevlar/epoxy composite. Materials Letters 59, 3287-3290 (2005)

486. Zheng, Y.J., Cui, L.S., Schrooten, J.: Basic design guidelines for SMA/epoxy smart composites. Materials Science \& Engineering A 390, 139-143 (2005)

487. Zheng, Y.J., Schrooten, J., Cui, L.: Thermomechanical properties of $\mathrm{TiNiCu}_{12}$ wire reinforced Kevlar/epoxy composites. Intermetallics 13, 305-308 (2005)

488. Zhou, G., Lloyd, P.: Design, manufacture and evaluation of bending behavior of composite beams embedded with SMA wires. Composites Science and Technology 69, 2034-2041 (2009)

489. Zhu, Y., Dui, G.: Effect of fiber shape on mechanical behavior of composite with elastoplastic matrix and SMA reinforcement. Journal of the Mechanical Behavior of Biomedical Materials 2, 454-459 (2009) 